\title{
Françoise Ozanne-Rivierre et Jean-Claude Rivierre, deux linguistes engagés auprès des Kanak et de leurs langues
}

Françoise Ozanne-Rivierre and Jean-Claude Rivierre, two linguists committed to the Kanak people and their languages

Isabelle Leblic

\section{(2) OpenEdition Journals}

Édition électronique

URL : https://journals.openedition.org/jso/12282

DOI : $10.4000 /$ jso. 12282

ISBN : $1760-7256$

ISSN : $1760-7256$

Éditeur

Société des océanistes

Édition imprimée

Date de publication : 31 décembre 2020

Pagination : 125-158

ISBN : 978-2-85430-139-7

ISSN : 0300-953x

Référence électronique

Isabelle Leblic, «Françoise Ozanne-Rivierre et Jean-Claude Rivierre, deux linguistes engagés auprès des Kanak et de leurs langues ", Journal de la Société des Océanistes [En ligne], 151 | 2020, mis en ligne le 01 janvier 2021, consulté le 30 janvier 2023. URL : http://journals.openedition.org/jso/12282 ; DOI : https://doi.org/10.4000/jso.12282

\section{c) $(1) \Theta$}

Creative Commons - Attribution - Pas d'Utilisation Commerciale - Pas de Modification 4.0 International - CC BY-NC-ND 4.0

https://creativecommons.org/licenses/by-nc-nd/4.0/ 


\section{Françoise Ozanne-Rivierre et Jean-Claude Rivierre, deux linguistes engagés auprès des Kanak et de leurs langues}

par

Isabelle LEBLIC

Je n'ai pas voulu ici faire un in memoriam classique sur les Rivierre - nous en avons eu dans nos colonnes pour chacun d'entre eux (voir Dépt. Océanie, 2007 et Saussol, Leblic ou Bensa, 2018) -, mais plutôt une biographie à travers la présentation de leurs carrières et de leurs apports, tant en linguistique océanienne que dans leurs engagements pour l'enseignement des langues kanak et la défense des Kanak qui, dès les années 1980, intensifièrent leurs revendications à l'indépendance et à la reconnaissance de leur identité et donc de leurs langues. La présentation de leur carrière permet aussi de retracer une partie de l'histoire administrative et matérielle des conditions de la recherche en ce dernier quart du $\mathrm{Xx}^{\mathrm{e}}$ siècle et au tournant du XXI ${ }^{e}$ siècle, et c'est donc aussi l'histoire de nos institutions que je présente ici à travers eux.

Françoise Ozanne-Rivierre (ci-après FOR) nous a quittés le 11 novembre 2007 à l'âge de 66 ans (moins de deux ans après son départ à la retraite), des suites d'une longue maladie et, un peu plus de dix ans plus tard, ce fut le tour de Jean-Claude Rivierre (JCR), le 5 janvier 2018 (à la retraite depuis décembre 2003), décédé subitement chez lui. Nous avons ainsi perdu deux amis, deux de nos collègues du LACITO CNRS où ils ont fait toute leur carrière, qui ont marqué fortement la linguistique océanienne et la recherche sur la Nouvelle-Calédonie, notamment sur les langues kanak, auxquelles ils ont consacré toute leur vie. Nous souhaitons, par ce volume, leur rendre hommage, tant leur parcours rempli d'humanité et leurs

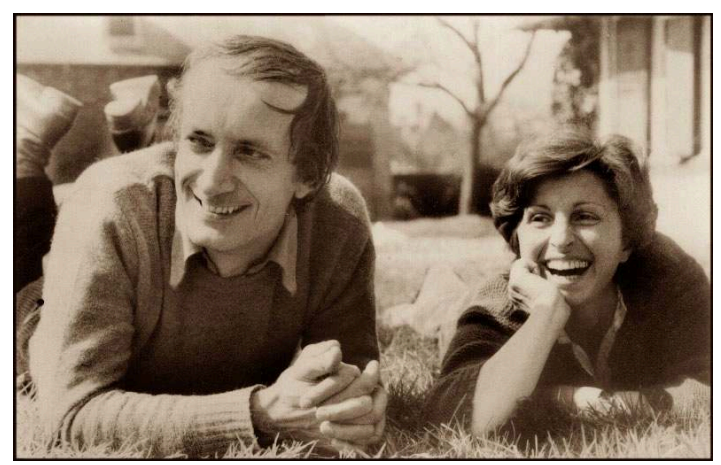

Рното 1. - Jean-Claude et Françoise (cliché CFR ${ }^{1}$ )

personnalités bienveillantes ont imprégné aussi bien les Kanak avec lesquels ils ont fait un grand bout de chemin que les générations de chercheurs qui ont suivi leurs traces; j'en fais partie à plus d'un titre, même si je ne suis pas linguiste.

Françoise et Jean-Claude sont tous les deux originaires de Normandie. Ils se seraient rencontrés à Caen où ils ont fait une partie de leurs études universitaires avant de poursuivre tous deux à Paris.

Mariés en juillet 1962, ils sont partis pour la première fois en Nouvelle-Calédonie à la demande d'André-Georges Haudricourt (AGH) ${ }^{2}$ qui cherchait un linguiste pour étudier les langues à tons de Nouvelle-Calédonie, ce que fit Jean-Claude pour le cèmuhî de Touho. Jeune recruté au CNRS fin 1964, accompagné de sa femme et de leur premier fils $\mathrm{Ni}$ colas alors âgé d'un an, ils débarquent du paquebot

1. Je remercie ici Nicolas Rivierre qui nous a donné accès à tous les clichés notés CFR pour « coll. familiale Rivierre ».

2. André-Georges Haudricourt (1911-1996) a été tout à la fois linguiste, botaniste, géographe et ethnologue français avec une formation d'ingénieur agronome, membre du CNRS-LACITO où il termina sa carrière comme directeur de recherche. Il fut à l'origine du développement des recherches linguistiques sur les langues de Nouvelle-Calédonie.

* Anthropologue, directeure de recheche LACITO CNRs, isabelle.leblic@cnrs.fr 


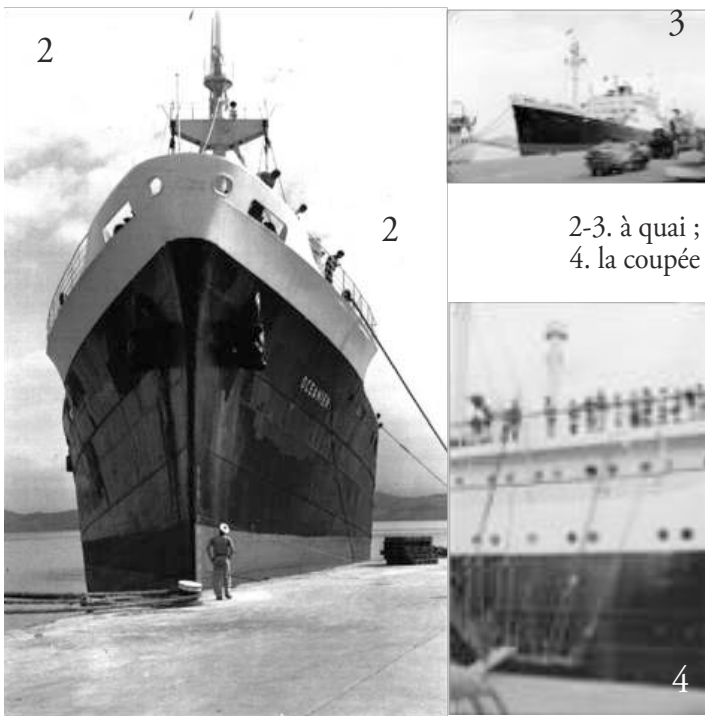

Рнотоs 2-4. - L'Océanien à Nouméa en mai 1965 (clich Alain de Bressy, http://www.messageries-maritimes.org/ oceanienB2.jpg

mixte L'Océanien IP à Nouméa le $1^{\text {er }}$ janvier 1965. Ils sont accueillis par Jean Guiart ${ }^{4}(\mathrm{~J} G)$ qui les confie à Alain Saussol, présent sur le terrain depuis trois semaines (Sausol, 2018: 611). Début 1967, Froinçoise (alors enceinte) et son fils Nicolas rentrent en France. Contrairement à ce que nous pensions, à en croire les ordres de mission consultés, il semblerait que JeanClaude ne soit revenu en France qu en juin 1967, soit qu'il en soit, son retour se fit après plus de deux an de terrain en Nouvelle-Calédonie (essentiellement Touho) et au Vanuatu. Il faut dire que les voyages par voie maritime imposaient de rester assez longtemp sur le terrain car on ne partait pas aussi facilement que de nos jours, où le voyage en avion ne dure qu'une journée et ne comporte qu'une seule escale. Lurs de jors il fallait encore compter plus d'une journée de traje avec de multiples escales.

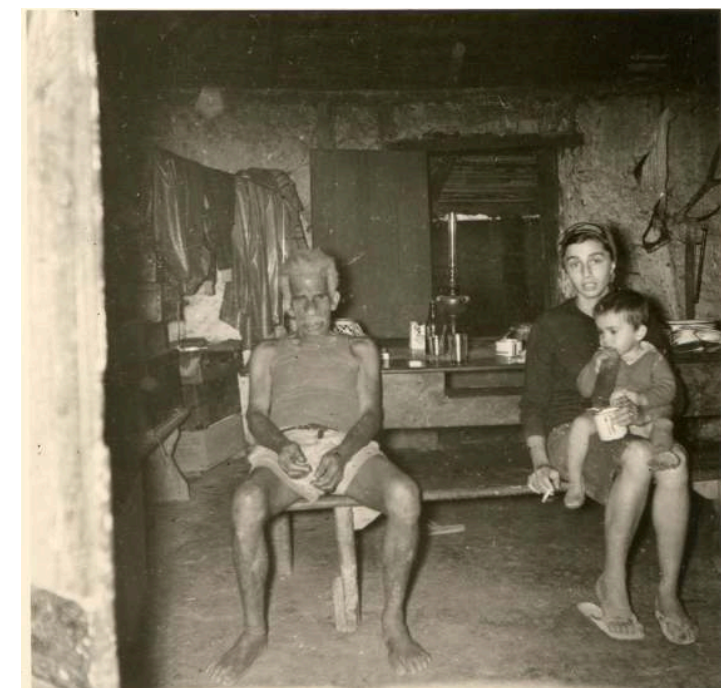

Рното 5. - Atéa Bouillant, le chef de la tribu des Poyes (Touho), et Françoise Ozanne-Rivierre (avec, sur ses ge(Touho), et Françoise Ozanne-Rivierre (avec, sur ses genoux, Nicolas, agé dun peu plus d un an), visibles
dans la case d'Atéa ( $c$. janv.-mars 1965 , cliché CFR)

Partis de Marseille le 23 novembre 1964, ils débarquèrent donc à Nouméa début janvier 1965, après un voyage d'environ cinq semaines. ${ }^{6}$. De janvier à juillet 1964, ils vécurent en Nouvelle-Calédonie essentiellement dans la région de Touho (Teganpaik, Poyes, Kowèi, Pwobëi, Vieux-Touho). Puis, du 1 août 1965 au 31 janvier 1966, la mission se poursuivit aux Nouvelles-Hébrides où ils firent l'inventaire des langues pour la RCP 27 (recherche coopérative sur vocabulaire et de textes ${ }^{7}$. Du $1^{\text {er février }} 1966$ à juin 1967 (Françoise et Nicolas étant rentrés début 1967) Jean-Claude poursuivit sa mission en NouvelleCalédonie et étudia les langues à tons de l'extrême sud (Goro, Touaourou, Unya et Païta, à partir du lexique d'Haudricourt qu'il porta de 1200 à 3500 mots pour Goro et Unya); il y recueillit aussi une vingtaine de contes et de récits (800 p. manuscrites).
3. L'Océanien II, ex-Nordam, a été affrété du 2 août 1963 à février 1967. « Lancé en 1938 à Rotterdam sous le nom de Noordam pour le compte de la Holland Amerika Line », il fut loué « aux Messageries en août 1963 pour remplacer Le Melanessien. Affecté à la (http://www.messageries-maritimes.org/oceanienB.html).

4. Jean Guiart (1925-2019) était ethnologue, disciple de Maurice Leenhardt et spécialiste de la Nouvelle-Calédonie (voir Saussol, 2019 ; Coiffier, 2019 et un prochain dossier du JSO sur Jean Guiart : L'ethnographie comme marathon d'une vie, coordonné par Lamon

5indstrom et Marc Tabani (https://journals.openedition.org/jso/11247).
Et non pas dès mai 1967, pour la naissance, comme nous l'avait indiqué Nicolas Rivierre (comm. pers., in Saussol, 2018 : 219) 6. Selon Alain Saussol (comm. pers.), « avec un cargo des Messageries maritimes, le Vanoise, par Suez, de Marseille à Nouméa, j’a mis cinquante jours. Pour être précis, on a quitté Marseille le soir du 19 octobre et on est arrivé à Nouméa le matin du 7 décembre. était rapide mais on a relâché plusieurs jours dans certains ports pour décharger puis charger, ce que ne devait pas faire un paquebot.
Généralement, on arrivait le matin (ou dans la nuit) et on repartait en fin de journée. C'est ce que devait faire en principe un paquebot. Un cargo pouvait rester plusieurs jours. Si je fais le décompte des escales du Vanoise, on a passé à quai : Gênes : 3 jours ; Diibouti : 1 jour ; Cochin : 2 jours; Colombo $: 2$ jours ; Perth $: 1$ jour ; Adéläide $: 1,5$ jour ; Melbourne + Sydney $: 9$ jours à quai. Cela donn donc 19-20 jours d'escale, donc les jours de navigation sur l'itinéraire représentent un mois. L'Océanien n'était pas un paquebot mais, comme le Calédonie et le Tahitien, des cargo-mixtes embarquant marchandises et passagers. Mais leur trafic devait se limiter aux pos sessions françaises: sur cette ligne, Djibouti et Noumea. Donc, avant Noumea, en route, ils s arretaient peu et leur trajet devait dure

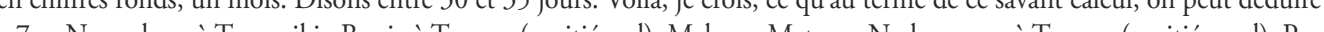
7. Na-makura à Tongariki ; Bunig à Tongoa (moitié sud), Makura, Mataso ; Na-kanamag à Tongoa (moitié nord), Pwele, nguma Emau, Emae ; langue polynésienne au village de Makata à Emae, à Port-Vila (Efate) ; étude de la langue à Erakor, Eratap, Pango à transcrits, copie de doc, missionnaires (100 p. dactylographiées) ; langue d'Aneityum.

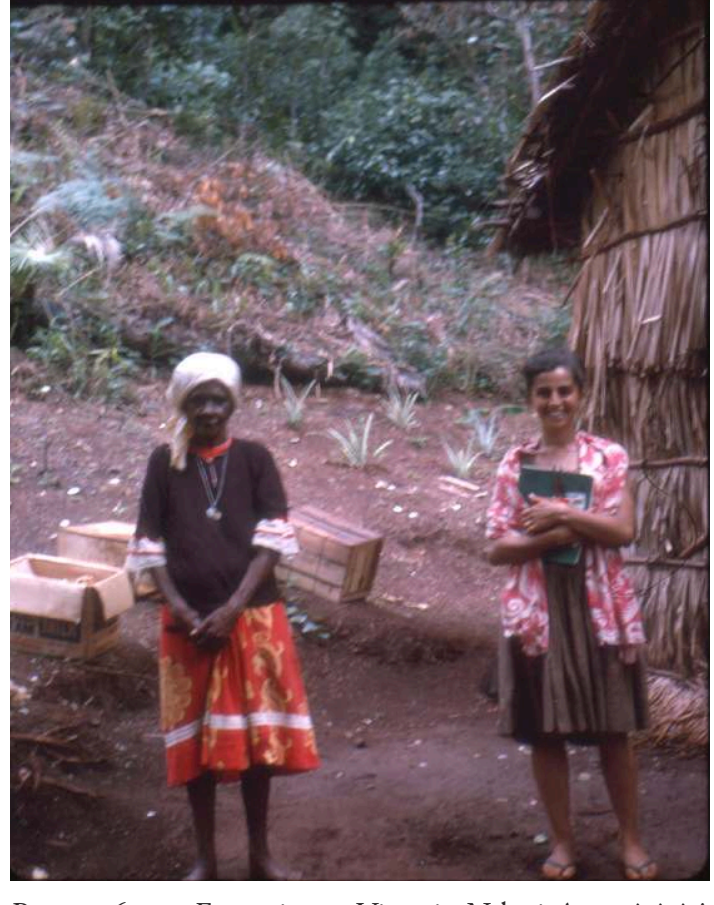
Pното 6. - «Françoise et Victoria Ndapi Angwéréré
Goro », Nouvelle-Calédonie (avril 1966) (cliché CFr)

Une rencontre importante dans bien des carrières

J'ai rencontré Jean-Claude et Françoise lorsque j'étais étudiante en ethnologie à Paris 5 - René Descartes, dans les années 1977-1978. À l'époque, je n'étais pas encore engagée sur le terrain calédonien, mais prévoyais, pour ma maitrise (soutenue en juin 1979), de partir au Pérou avec un ethnologue péruvien qui était leur voisin de bureau dans les locaux du CNRS à Ivry où le LACITO était alors hébergé. Je n'ai plus en mémoire la raison de notre rencontre dans leur bureau à Ivry. Mais je me souviens quils mavaient prévenue que la

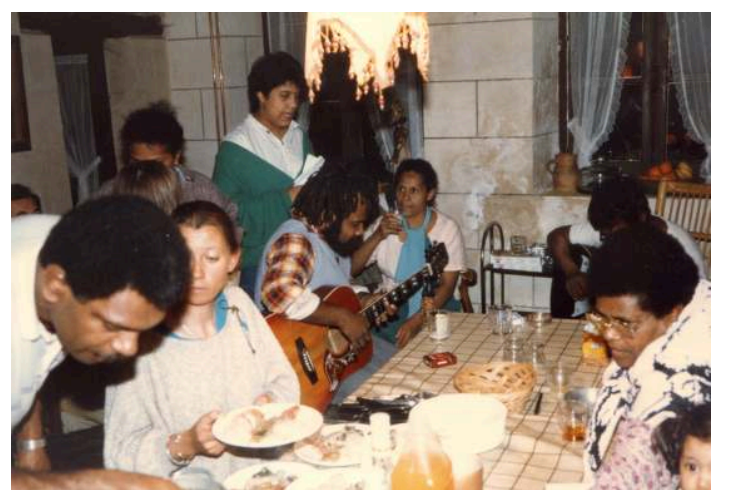

Рното 9. - Une soirée à Retheuil dans la maison des Rivierre où j'étais présente (27 avril 1987, cliché CFR)

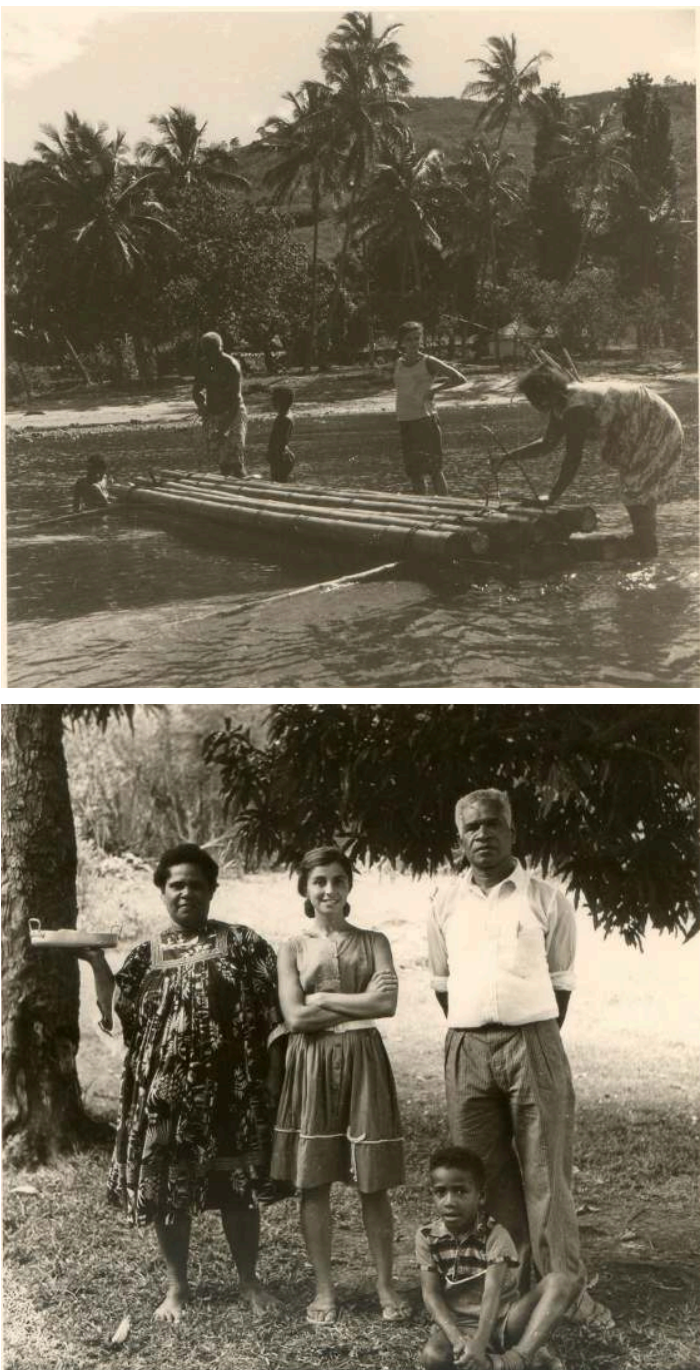

Pнотоs 7-8. - Françoise regardant un couple en train de s'affairer sur un radeau (en haut) et portrait de groupe (Ters.) , ces photos sont bien prises à Touho, car, dans pers.), "ces photos sont bien prises a louho, car, dan
les années 1965-1966, il y avait bien des radeaux ; mais j’ai du mal à reconnatre l'homme et la femme qui entourent Françoise sur la photo du bas "

avec laquelle je devais partir n'était pas forcément fable...et ils eurent raison ${ }^{8}$ Quelques années après, en cours de thèse de doctorat, toujours sur l'île de Molene, je cherchis un tertain plus lointain , de lane but, je minscris à "inalco en DuLCo" de $\rightarrow$ rohi de Tahiti et de apprs Suite à de Tahiti et de drehu, langue de Lifou. Suite à ma rencontre avec Jacques Barrau (Leblic, $2002: 115)$, je démarrai des recherches sur les pêcheurs kanak du sud de la Nouvelle-Calédonie et, ce titre, Jean-Claude et Françoise me firent entrer au LACITO en 1982. Et, comme eux, une fois admise au

8. Inscrite également à l'université de Vincennes Paris 8 en histoire contemporaine de l'Amérique latine, en espagnol et en quechu débutant, j'avais travaillé pour lui bénévolement sur un dictionnaire quechua afin de préparer mon départ qui devait me permettre lorsque le voyage fut prévu, je nen fis pas partie et fus donc obligée de trouver en urgence un autre terrain pour réaliser mon enquête de maîtrise. C'est ainsi que je réalisai ma première enquête de terrain à Molène. Voir aussi Leblic (2010 et 2018).

9. Diplôme universitaire de langues et civilisations orientales préparé en deux ans, de septembre 1980 à juin 1982. 
CNRS en 1991, j'y fis toute ma carrière et m'y trouve encore, à quelques années de la retraite.

Ce fut donc un long parcours à leurs côtés, mélangeant amitié, recherche, engagement aux côtés des Kanak et même séjours de terrain en partie communs. D'ailleurs, en 1986, Jean-Claude est venu me voir sur le terrain à Goro et nous avons revu me voir sur le terrain a Goro et nous avons revu Isaac Vendégou à Touaourou.

J'ai participé aux travaux du groupe Océanie au sein du LACITO de nombreuses années durant, à une époque où lanthropologie occupait une place plus importante dans notre laboratoire et où nous étion organisés selon des aires linguistiques et culturelles (Leblic et Masquelier, 2020). Nous y avons men notamment des recherches sur les vocabulaires de parenté ce qui me donna l'occasion ve traviller de pacen plus ćtrite avec les deur Rivierre et, plus po çulièr thentus pal's terminologies du sud end a des Pins et externe sud de la Grande Terre (Goro) furent mes premiers terrains kanak (1983 \& 1985-1986 pour un total de dix-huit mois, voir Leblic, 2018a). Ces materiaux ont enrichi la presentation que Françoise a faite lors du colloque Études des sociétés kanak (ESK) où nous avons rendu compte de nos recherches, financees en partie par ce programme de recherche demandé par Jean-Marie Tjibaou et, à la requête de ce dernier, piloté par Daniel de Coppet (voir OzanneRivierre, 2000a pour les terminologies de parenté kanak et Bensa et Leblic éds, 2000 pour un aperçu des recherches ESK)

\section{Le programme ESK}

Le programme "Études des sociétés kanak » avai été conçu en 1988 par Jean-Marie Tjibaou dans le cadre des accords de Matignon qui prévoyaient auss la création de l'Agence pour le développement de la culture kanak (ADCK) et la construction d'un centre culturel, devenu le centre culturel Tjibaou (CCT) Dans l'esprit de Jean-Marie Tjibaou, ce programme de recherche, en aidant à réfléchir sur le monde kanak encore très mal connu par la société coloniale, devait de jeunes futurs chercheurs kanak que nous aurions contribués à former et qui auraient pu assurer pas pas le cas et ce programme ne benéficia pratiquement qu aux chercheurs et étudiants métropolitain au titre de la linguistique ; j'y étais, avec d'autres, a titre de l'anthropologie...

Ce programme ESK, pluridisciplinaire, a permis outre des études ethnologiques, le développement de plusieurs thématiques de recherche en histoire, géographie, archéologie, musicologie, sur plusieurs répermettre d'associer les chercheurs métropolitains contribués à former et qui auraient pu assurer releve et prendre en charge lavenir de la recherch Jean-Claude Rivierre était membre du conseil d'ES

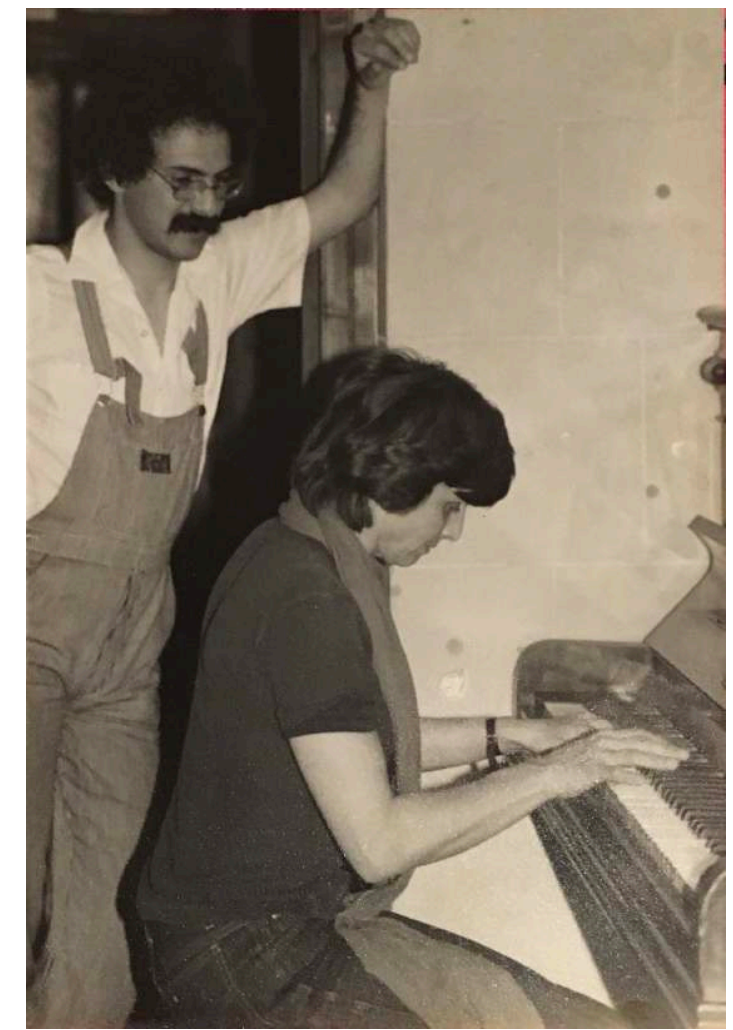

Pнто 10. - Francoise Ozanne-Rivierre joue du piano en fin de soirée à Retheuil le 1er mai 1983 (cliché Leblic)

gions de Nouvelle-Calédonie (Arama, Koné, Canala, Maré, Lifou, Ponérihouen, etc.). Mis en œuvre institutionnellement par les ministères des Départements et Territoires d'Outre-mer et de la Culture (Mission du Parimoine ethnologique) qui en assurèrent le financement, son suivi et sa mise en œuvre furen confiés dans un premier temps au CNRS, à Daniel de Coppet et à l'équipe qu'il dirigeait alors, ERASME, la demande de Jean-Marie Tjibaou. Après le décès de ce dernier, il fut récupéré par l'eHEss en 1993 , sous la responsabilité de Marc Augé puis de Jacques Revel, en leur qualité de présidents de l'eHess. Le conseil scientifique était composé d'Alban Bensa de Daniel de Coppet (eness) ; Jöl Bonnema (овстом) ; Jean Freyss (Univerité Pais I) Pas. Godin ; Pierre Culland, Gérard Ermisse, Alain Morel et Bernard Gilman (ministère de la Culture) Isabelle Leblic, Claire Moyse et Jean-Claude Rivierre (LACITO CNRS); Géraud de Gallard (DOM-тOM) Wassissi Konyi, Emmanuel Kasarhérou et Octave Togna (ADCr) ; Roger Boulay (musée des Arts afridains et océaniens). La plupart des études retenues dans le volume étić par Bensa et moi-même (2000) avaient fait l'objet de communications au colloque "Études des sociétés kanak, systèmes sociaux en devenir " organisé en octobre 1995 à Paris (voir Bensa et Leblic, $2000: 2-3$ ).

10. Je me souviens notamment de séances de travail le week-end dans leur maison de campagne mêlant moments studieux et détente, avec balades en forêt, cueillette de champignons à l
mettait au piano pour terminer la soirée. (voir photo 10).

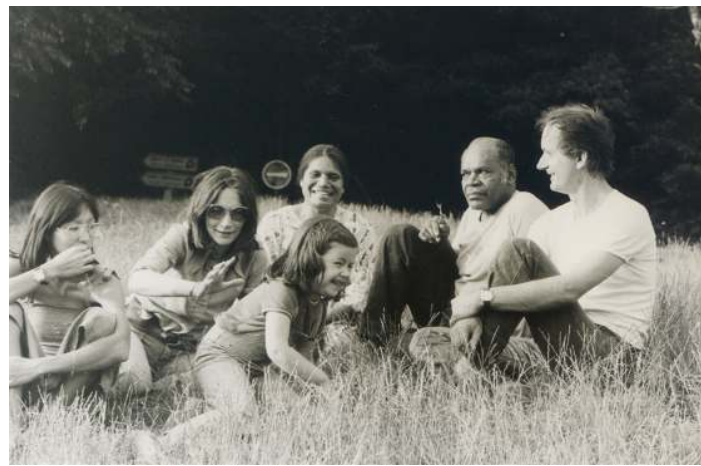

Рното 11. - Balade en forêt à Retheuil. Jean-Claude Rvierre avec Françoise Peeters du LACITO (et sa fille) avec le couple de Kanak dont ils parlent ci-dessous, identifé Goromido et sa femme Élise (1978) (cliché crn)

Linvestissement de deux vies au profit des langues et cultures kanak et de leur enseignement

Françoise et Jean-Claude Rivierre ont toujours eu à cœur de mener de front recherches fondamentales et recherches appliquées. Très tôt, au début des années 1980 , ils ont tout fait pour donner une place aux langues kanak dans l'enseignement du territoire en élaborant des matériaux à visée pédagogique.

En 1976, André-Georges Haudricourt, directeur de recherche de Françoise, précise, dans l'évaluation de sa recherche annuelle, que :

"[Françoise] a pris l'heureuse initiative de faire une publication provisoire ronéotee [303 p.] de sa récolte textes de litterature orale de façon a ce que les inform teurs puissent, à leur tour, en disposer, ce qui facilitera

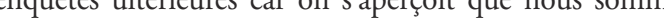
bien loin d'avoir récolté le principal de cette littérature orale. "

Françoise note en effet dans son rapport d'activité ${ }^{1}$ de 1976 que :

" en région nemi, j’ai pu distribuer à mes informateurs des exemplaires des textes nemi multigraphiés en 197 ainsi que deux photocopies du projet de dictionnaire alphabétique nemi élaboré en début d'année. L'intérêt suscité par ces premiers résultats a été évident. Les retombées du Festival Melanesia 2000 qui sest déroulé à Nouméa en 1975 sont encore très sensibles. On sent que cette manifestation culturelle, dont I un des organisateurs est luimême originaire de Hienghène [Jean-Marie Tjibaou], réveillé chez de nombreux Mélanésiens un profond intérèt pour leur propre culture. "

Cela répond aux soucis que nous avons tous de mettre à disposition des personnes avec lesquelles nous avons travaillé, des éléments concrets issus de nos enquêtes, ce qu'on appelait aussi le « retour aux enquêtés ". ans et demi) et à examen de l'équipe (à 4 ou 5 ans).

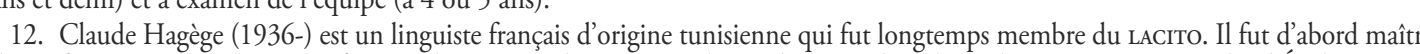
de conférences en 1970 puis professeur à l'u des hautes études en 1977, puis professeur titulaire de la chaire de théorie linguistique au Collège de France entre 1988 et 2006 ,
Dans son rapport d'activité 1977, Jean-Claude revient également sur sa façon de travailler : relecture avec les informateurs des textes devant être publiés en enrichissement avec eux de l'appareil de notes, méthode que j’applique également en anthropologie, en faisant relire au maximum mes écrits avant publication.

Montrant que le terrain en Nouvelle-Calédonie (comme ailleurs) induit, pour le chercheur, un certain son rapport d'activité de 1978, il précise aussi que :

"Mes mois d'été ont été particulièrement creux sur le plan du travail personnel, suite au départ de ma femme en tun couple de Néo-Clédoniens en congé, ces derniers ', charge de réciprocité, les accueillir à mon tour, les guider

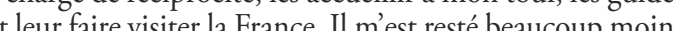
e temps que je ne le pensais pour leur utilisation éventuelle. Je n'ai transcrit qu'une centaine de pages de contes avec le jeune Hébridais (de langue na-makura); j’ai d'intéressantes séances de travail avec le couple calédonien ur la tradition orale en voie de publication. "

Ce qu'il ne dit pas ici, dans ce rapport qui reste administratif, c est la nature des relations amicales et chaleureuses qu il avait avec Elise et Antoine Goromido, comme avec nombre de ses interlocuteurs. aborations des liens d'amitié très forts, comme je l'a fait moi-même avec Clément Vendégou et sa famille à Goro, ou avec André, Gaby et Marthe Moentéapo (et toute leur famille) ou Déwé Gorodé, Marcel et Téâ Pourouin à Ponérihouen qui sont pour moi une part de ma famille.

Jean-Claude Rivierre évoque, dans son rapport de 1978, dans le paragraphe consacré aux « activités col"Vu l'évolution de la situation politique en Nouvelle Calédonie, l'enseionement des langues està l'ordre du jour. Deux instances officielles tentent de mettre en place un programme : - sur le plan local, l'assemblée territoriale cree un comité des langues vernaculaires dont je fais pardu ministere des TOM-DOM [sic] a été créé à l'automne, composé principalement de notre section [Océanie du LACITO]. La section Océanie du laboratoire disposant de la quasi-totalite de linformation sur ces langues, nous seron patement mis a contribution pour la materialisation de ce

Pour la session de printemps 1978, c'est Claude Hagège $e^{12}$ qui est désigné rapporteur par la section $d u$ comité national du CNRS pour Jean-Claude; il émet havis très favorable à son renouvellement. Dans so apport de 1978, Jean-Claude donne quelques informations sur son ATP (Action thématique programmée) nombre de retours vis-à-vis de ses interlocuteurs, dan tie ; - au plan métropolitain, un comité consultatif auprès 


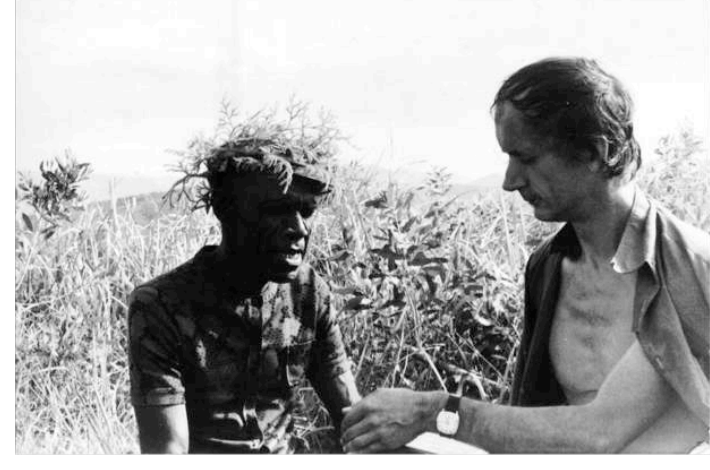

"Il est donc nécessaire, pour transcrire les langues de l'or lité, de disposer de caractères spéciaux qui permettent de rendre mental lorsquil s'sagit d'utiliser ces langues dans l'enseignement pour l'alphabétisation et pour le transfert de technologie... Une
écriture inadaptée aboutit à un rejet et à un échec de l'alphabétiécriture inadaptée aboutit à un rejet et à un échec de l'alphabét-
sation. » (Jacqueline M.C. Thomas, Panneau Expolangue 1984 sation. " (Jacqueline M.C. Thomas, Panneau Expolangue
https://lacito.cnrs.fr/expos/oralecrit/quelle_ecriture2.htm)

Рното 12. - « Quelle écriture (Pwöbei, Nouvelle-Calédonie) ? " (photo extraite du panneau Quelle écriture (2), de Jacqueline M.C. Thomas, de lexposition De Loral à lecrit, Expolangue 1984) : Antoine Oudodopoé Pwèhènèbé et Jean-Claude Rivierre en plein travail d'enregistrement
Pwöbei (vallée de la Tiwaka) en 1977 (cliché Bensa)

sur l'expression linguistique qui a démarré en 1978 (pour finir en 1980) avec le départ en mission d'A. G. Haudricourt et de F. Ozanne-Rivierre en Nouvelle-Calédonie et de M. Coyaud aux Philippines. Il signale que l'enseignement des langues vernaculaires de l'évolution de la situation politique du territoire. En 1979, Jean-Claude note, à la rubrique " alphabétisation " de son rapport d'activité, qu'il a contribué pour 40 pages au Manuel d'alphabétisation dans bue pour 40 pages au Manuel d'alphabetisation da méa, DEC) : "Présentation générale des langues de méa, DEC) : " Présentation générale des langues de Nouvelle-Calédonie ", et pour 60 pages au volume
de textes illustrant les langues et alphabets retenus et présentés dans le manuel (Nouméa, DEC) : « Exposés et textes sur les langues tonales du centre et du sud " :

"Comme prévu l'an dernier, la section a été sollicitée our l'élaboration d'un manuel d'alphabétisation dans les diverses langues de Nouvelle-Calédonie. La demande provient de la Direction de l'enseignement catholique. Le n doit sous presse a Noumea [cf. Haudricourt et al., 1979] ainsi que l'adoption d'une écriture aussi rationnelle ainsi que ladoption d une ecriture aussi rationnélle et unifiee que possible pour ces langues très diversifiees. Un
second volume, prêt pour l'impression, contient deux cent pages de textes illustrant les langues et alphabets retenus présentés dans le premier volume. » (Extrait du rapport de presentes dans
1979 de JCR)

Comme nous l'avons noté dans le récapitulatif de a carrière, l'année 1980 voit la poursuite des projets collectifs et personnels antérieurs. Jean-Claude précise, à propos des dictionnaires, limportance politique de l'enseignement des langues vernaculaires dans ce contexte colonial. Vers le milieu de l'année, apprend que le paicî va être enseigné, en raison d'un énième statut (le statut Dijoud, voir Leblic, 1993 : $28,55-57)$ :

«la politique Dijoud visant à calmer les revendications canaques (projet de réforme foncière - déjà sabotée -, et politique de promotion mélanésienne), politique qui rencontre beaucoup de méfiance, tant à l'extrême gauche que dans les milieux coloniaux. Les langues intéressent soudain beaucoup de monde et trois organismes (au moins) sont en compétition: la Direction de l'enseignemen catholique [DEC] (mise pour l'instant hors jeu par l'élimination du directeur), l'Institut mélanésien, soutenu pa ladministration mais à peine sorti des limbes, et le vicerectorat, bien doté en personnels et moyens et disposan un centre (territorial) de documentation et de recherches pédagogiques (CTRDP). Plusieurs enseignants sont affectés

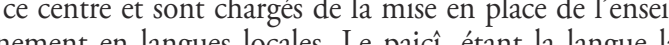
las plus parlée de la Grande Terre, a eté retenu en priorite métropole et que j'ai eue comme élève au Langues orientales, a été chargée de s'en occuper. Son Langues orienliéè à mon éventulle participation au projet et j'ai accepté immédiatement. Cette nouvelle politique in jai accepte un siècle et demi de rejet et d'ignorance des langues de la culture canaques; elle suscite beaucoun l'espoir d'intérêt thez les populations concernées. " (Extrait du rapport d'activité 1980 de JCR)

Très tôt, Jean-Claude et Françoise ont eu à cœur de participer aux projets educatifs locaux, comme ils firent par la suite dans les années 1980, en lien avec les indépendantistes kanak (supra). Dans son rapport d'activité de l'année 1980, Jean-Claude précise que

« Mes recherches s'avèrent être trop liées à celles d'autres chercheurs ou d'autres organismes pour qu'il me soit possible, comme par le passé, de distinguer entre recherches personelles et recherches collectives. Mon travail reste toujours centré sur la Nouvelle-Calédonie et je ferai éta ici de la progression de mes recherches dans les différents domaines ou je travaille. Ma preoccupation principal recuellie au cours de plusir à publier la documentation recuellie au cours de plusieurs années denquête. Lorientation générale de ma recherche est liée aussi à la transculturelle et politiou du monde málańsien inféchit le culturelle et politique du monde mélanésien infléchit travail en cours et impose un certain nombre de priorités.

Dans son rapport d'activité de l'année 1981, JeanClaude écrit d'ailleurs que :

"L'évolution de la situation politique en Nouvelle-Caédonie et les progrès de ce pays vers la voie de l'indépenance ont fortement inflechi lorientation des travaux des cerritoire d'outre-

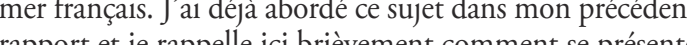
apport et je rappelle ici briverenent comment se présente es Néo-calédusquà une date très récene, les 28 langues soit dans l'enseignement dispenś sur le teritore ens lan lenslique (presse, littérature, médias, etc). Les evendications canaques sont particulièrement pressantes dans le domine culturel et le plan Dijoud mis en route en 1979 se fixait plusieurs objectifs en ce qui concerne la culture et les langues. Notamment : - un enseignement
- la réforme de l'enseignement primaire : prise en compte des langues parlées par les élèves; enseignement du français comme langue seconde. Notre contribution à ce programme a commencé par la publication d'une plaquette pour l'écriture des langues et d'un livre illustrant ces pro-

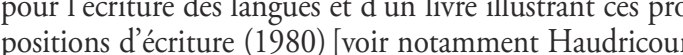
et al. (1979) ; Rivierre (1984)]. Puis, elle a pris l'an dernie des proportions plus importantes au point que, pour part, mes travaux sont à peu près tous destinés à faire avancer ces objectifs. » (Extrait du rapport de 1981 de JCR)

Ce côté « militant » pour la reconnaissance des langues kanak s'exprime ici clairement

«Le recueil de traditions publié utilise les alphabets proposés et montre que toutes les langues peuvent être écrites ce qui est loin d'être évident pour tout le monde sur place. Un autre recueil, publié par la Société d'études historiques de Nouméa, est entièrement en français et se présente comme "le livre de la conciliation". Nos travaux en langue vernaculaire avec mot à mot y sont jugés inutiles et "prétendument scientifiques". Ceci pour donner une idée de l'm lon

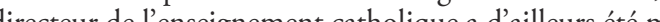
directeur de lenseigne à la porte depuis.

J'ai continué la préparation du livre de contes en langues paicî que je pensais publier en 1980 (en collaboration prép. Ber et in Cette prolongation du travil de prép sée lorsque j'ai appris que cette langue serait enseignée a sée litre expé j'i a ence Dans la seconde persective (étude de la tradition or Dour elle-même) Bensa pon vue de la publication le manuscrit du premien (500 p. environ), des traditions orales en langue de Touho, annoncés depuis fort longtemps. L'oung cound ho, an. Bensa puis les textes et leurs commentaires. Huit contes y sont passés au crible de l'analyse linguistique et ethnologique, pour dégager la symbolique utilisée et étudier les

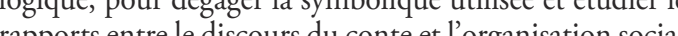
[voir Bensa et Rivierre, 1982]. Nous y montrons que ces

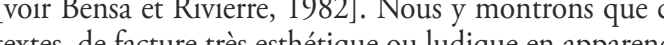
recèlent un message politico-historique riche et compexe. La préparation de ces textes s'est avérée trop difficile pour quil soit envisageable de continuer dans la même voie : qutres textes auront une présentation allégée à l'exce : des mythes concernant les lignages et les clans. Vu l'én mité du corpus et la difficulté du sujet plusieurs ané

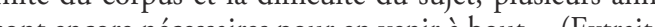
rapport de 1980 de JCR)

Dans son rapport de 1980, il signale également que les traditions orales "peuvent être publiées comm illustration des travaux linguistiques avec un appareil de notes réduit à l'essentiel, ou constituer un objet d'études en soi ". Un petit recueil de textes, en huit langues, publié à six mains (Françoise et Jean-Claude avec Claire Moyse-Faurie) illustre la première perspective dont il est question ici (Rivierre et al., 1980) :

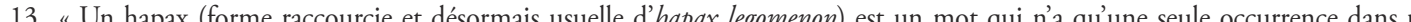
corpus donné. " (fr.wikipedia.org > wiki > Hapax).
"Ce recueil fait suite au travail demandé par la Direcion de l'enseignement catholique de Nouméa en 19 de publié également cette année à Nouméa : les langues (signé : section Océanie du LP3-121 Vvoir Haudricourt

Désireux de donner accès à ses recherches à toute personne qui, en Nouvelle-Calédonie, s’intéresserait aux langues et cultures kanak, Jean-Claude mit disposition l'ensemble de ses données de l'époque, comme il le précise à propos de sa mission qui eut entièrement consacrée à la langue paicî :

"Mon travail a été grandement facilité par l'active participation des informateurs, par le soutien logistique documentation péda à qui ;'ai fourni en retour la totalité de ma documentation (fichiers, textes, bandes mannétiques). J'ai beaucoup précié le żle et le sérieux de toutes les personnes eng gées dans ce projet auquel je participe sans me sentir liéd facon exclusive avec qui que ce soit. J'ai également participé à des séances de travail avec du personnel de l'enseignement catholique et de guement catholique et de l
du rapport de 1980 de JCR)

Le paicî étant devenu la première langue d'enseignement dans le secondaire, Jean-Claude recentra ses activites de recherche, missions comprises, sur cette langue :

«Le nouveau cadre dans lequel je travaille fait que mes publications seront tout de suite utilisees dans l'enseignemire préparé quantité de mots poétiques ou hapax ${ }^{13}$, ces créations sont fort intéressentes à étudier pour elles-mêmes

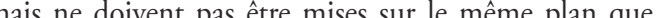
Ocabuhire courant uilisé à ce stade. J'ai retravillé sur les nomenclatures du monde naturel (coquillages, poissons, oiseaux... [... J'ai décidé de laisser de côté les poésies trop difficiles à traduire et à présenter, pour m’occuper uniquement des textes en prose dont j'ai maintenant terminé la dactylographie. Une fois tapés, ces textes ont été transmis au CTRDP et utilisés immédiatement dans l'enseignement. Leur publication nécessitera un travail ethnographique préalable. Il résulte de ce travail que le CTRDP dispose dès maintenant d'un important matériel pédagogique (lexique, 450 pages de textes, nomenclatures, etc.) et que tions. Nous travaillons en étroite collaboration pour élaborer des outils complémentaires, harmoniser nos méthodes et nos conventions. À titre d' exemple, le dictionnaire que e publie cette année contient : - la notation systematique des tons; - la traduction en français ; - l'analyse morphematique des tres nombreux dérives et composes. Le dictonhaire actuellement en cours de elaboration au CTRDP partir du mien utilise la même écriture mais : - il est monoingue ; chaque terme est expliqué et défini dans la langue, sans un mot de français ; - il est écrit plus simplement, sans décomposer systématiquement les mots pour éviter prolifération des traits d'union ; - les tons ne sont pas notés puisque cette suppression s'est avérée possible en expresal., 1979]). " (Extrait du rapport de 1980 de JCR) 
En 1982, Françoise indique également, dans son rapport d'activité, qu'une des orientations qu'elle poursuit est la recherche appliquée en lien avec la mise en place d'un enseignement dans les langues kanak en Nouvelle-Calédonie. Et, comme l'ensemble des chercheurs de la section Océanie du LAciTo, elle est engagée dans le programme DGRST d' «études des langues néo-calédoniennes, restitution des résultats, applications pédagogiques, formation à la recherche ».

«La nouvelle politique culturelle qui se développe acmine de plus en plus nettement vers la voie de l'indemine de plus en plus nettement vers la voie de l'indépendance) et surtout la mise en place d'enseignements lans les langues vernaculaires ont profondément infléch laboratoire. » (Extrait du rapport de 1982 de FOR)

Elle revient aussi sur la question de l'adoption de conventions d'écriture, suite à la publication en 1979 de la plaquette sur les langues comportant des propositions d'écriture. Elle évoque également l'ensegnement du iaai (langue d'Ouvéa) qui est prévu à la rentrée scolaire de janvier 1984. La question est ici quelque peu différente car le iaai, comme toutes les quelque pecu dén une grande tradition d'ecriture missionnaire protestante. Françoise a don tenté de concilier sa propre ecriture et celle en usage Ouvea, en proposant a cette dernière les modification sur les points où lecriture missionnaire est lacunaire. En application de la méthode en usage, elle doit revoir son dictionnaire avant publication sur place, lors de prochaine mission à Ouvéa, en janvier-février 1983 notamment avec des enseignants mélanésiens :

" Je compte avec eux discuter des aménagements proposés en matière d'écriture et du problème du découpage des tiles je l'espère, leur servir de point de déprt à l'ce, pourra d'un dictionnaire monolingue iaxi sur le modè de ceur que préparent en ce moment les enseignants-chercheurs mélanésiens du cTRDp de Nouméa en paicî (langue de Grande Terre) et en drehu (langue de Lifou). "(Extrait du

Dans son rapport 1982-1983, elle précise que :

"Les traditions protestantes d'écriture ont été autant que possible respectées. Quelques aménagements on liques, introduction de quelques signes nouveaux pour noter des différences qui n'étaient pas prises en compte dans l'orthoraphe traditionnelle. Pour régler le problème (important en iaai) du découpage des mots, j'ai adopté, dans le dictionnire, un système de renvois évitant ainsi prolifération de traits d'union qui suscitaient, à juste titre, brircoup de réticence chez les locuteurs iaii. " (Extrait du rapport 1982-1983 de FOR)

En 1985, Jean-Claude Rivierre participe au volume des Temps modernes sur l'indépendance kanak avec u texte sur la politique linguistique et l'enseignemen des langues, rédigé plusieurs années auparavant et repris pour faire une version française remaniée l'article paru en anglais dans la revue de linguistique de Port-Moresby (c. 1981). Plus tard, il publia avec
Francoise Ozanne-Rivierre et Claire Moyse-Faurie e interview de Marie-Adele Jorédié sur l'expérience de l'école populaire kanak (EPK - cf. doc. 1-3) et le problème de 'enseignement des langues en Nouvellelédonie (1987).

Le couple a donc initié plusieurs programmes de recherches et de diffusion des connaissances à destination du "pays " - c'est ainsi que les Kanak nomment la Nouvelle-Calédonie - dont un ensemble de publications échelonnées de l'équipe Océanie du LACITO pour le ministère des DOM-TOM, pour le projet DGRST de 1982, etc.

En effet, le programme DGRST de trois ans, intitulé Étude des langues néo-calédoniennes. Applications pédagogiques restitution des résultats, formation tions pédagogiques, restitution des resultats, formatio a la recherche en réponse a lappel d' offre "Sciences humaines et économiques dans les TOM-DOM [sic] ", débuta en 1982. Outre l'intensification des recherches sur le terrain, il était basé sur deux objectifs : 1 . " disposer, dans un délai de quatre à cinq ans, de travaux de qualité couvrant à peu près l'ensemble des langues néocalédoniennes " et 2. "application pédagogiques, restitution des résultats et formation à a recherche ". Il prévoyait :

"la réalisation de monographies et de descriptions consacrées à des langues encore insuffisamment connues. Ceci celoppement de maine grammatical ou phonologique. - Mettre sur pied des enseignements de langues au niveau du secondai ou du primaire et développer notre colla du secondaire organismes qui depuis peu s'intéressent aux langues et à organismes qui depuis peu sinteressent aux langues et à la tion pédagogique (CTRDP) dépendant du vice-rectorat, l'Institut mélanésien. Le programme a été officiellement accepté et nous sommes encore encouragés de ce fait à nou soucier des applications et des prolongements pratiques $d$ nos recherches. " (Extrait du rapport 1981 de JCR)

Dans son rapport d'activité 1982-1983, JeanClaude précise à nouveau que, durant ces deux dernières années, ses travaux ont été effectués :

"dans le cadre de deux programmes financés par la DGRST et le ministère des DOM-TOM; ces programmes cos recherches et des échénces qu'elle doit respecter. I'impulsion de ces programmes par des organismes officiels témoigne des mutations en cours en Nouvelle-Calédonie qui a de jours statut de territoire d'outre-mer francais. Alors que nos recherches se sont déroulées dans une certaine indiférence jusqu'en 1978 environ, la poussée du mouvement indépendantiste et les revendications culturelles exprimées inder place par les Mélanésiens ont amené le gouvernemen français à développer des actions en faveur de la culture et des langues canaques, qui nous concernent bien évidemment. " (Extrait du rapport 1982-1983 de JCR)

Pour l'année 1984, en plus de la préparation de l'exposition Expolangue, Jean-Claude note que

"Compte tenu des événements de ces derniers mois, les missions de recherche linguistique en Nouvelle-Calédonie sont suspendues pour une durée indéterminée. [...] J’ai contribué comme précédemment aux travaux concernant plusieurs langues de la côte est de la Grande Terre : séances de travail avec la responsable de l'enseignement catholique présente à Paris pendant le premier trimestre ; publication de textes en langue paicî: poèmes, récits et contes in jè nye wii mwara paicî et jè nye pi-cöö mwara paicî llivret de l'enseignant) publié par le CTRDP de Nouméa. " (Extrait du rapport 1984 de JCR)

J'ai eu l'occasion de me retrouver sur le terrain en même temps qu'eux à plusieurs reprises à cette période : je suis retournée avec Jean-Claude à Yaté en 1986 ; avec Françoise et lui, jai participé à diverses rencontres sur le terrain sur la côte est, à Pouébo à la fin des années 1980 où Françoise se trouvait alors pour assister à une kermesse locale (où j'ai eu l'occasion de croiser, entre autres, Scolastique Pidjot, une de ses collaboratrices et amie). J'étais également à proximité de Poindimié quand ils ont réalisé, à la demande de la région Nord, l'un des stages d'écriture des langues, celui du paicî et cèmuhî̀ à destination des enseignants, pour lesquels ils avaient réalisé des abécédaires pour servir de matériel pédagogique dans les écoles (fevrier 1986) ${ }^{14}$. En effet, la régio Nord, instituée en 1985 par la loi Fabius-Pisani (voir Leblic, 1993 : 64),

« reconnaissait des prérogatives importantes aux régions en matière éducatives et ménageait des possibilités d'interventions exterieures dans les ecoles publiques pour tout ce qui touche a la vie culturelle et aux langues, totalement ignorees jusqu a cette date. J ai assure quatre stages durant cette période, sur la côte est et sur la cote ouest, pour les deux langues numériquement les plus parlées, le paicî et le cèmuhî. Initialement destinés aux intervenants dans les écoles, ces stages ont été suivis par un public plus large de jeunes désireux avant tout de travailler sur leur langue et leur culture. Compte tenu de la ferveur des participants, nous avons travalle dans de bonnes conditions à la réalisation des objectifs que nous nous étions fixés : diffusion des méthodes décriture et de lecture, élaboration de fiches pédagogiques et de documents en langue vernaculaire su tous les aspects de la vie traditionnelle (milieu nature, technologies, traditions orales, etc.), formation d'ani teurs-enseignants." (Extrait du rapport 1985 de JCR)

Durant cette mission qui se déroula du 20 janvier au 31 mars 1986, Francoise et lui ont donc conduit et animé huit stages d'initiation à la linguistique et de formation d'intervenants dans les écoles publiques, avec élaboration de matériels pédagogiques qui ont connu un grand succès et accueilli 120 stagiaires (fiche documentaire ${ }^{15}$ de 1986). Jean-Claude Rivierre avait en charge les stages pour former les locuteurs à l'écriture et à l'enseignement du paicî et du

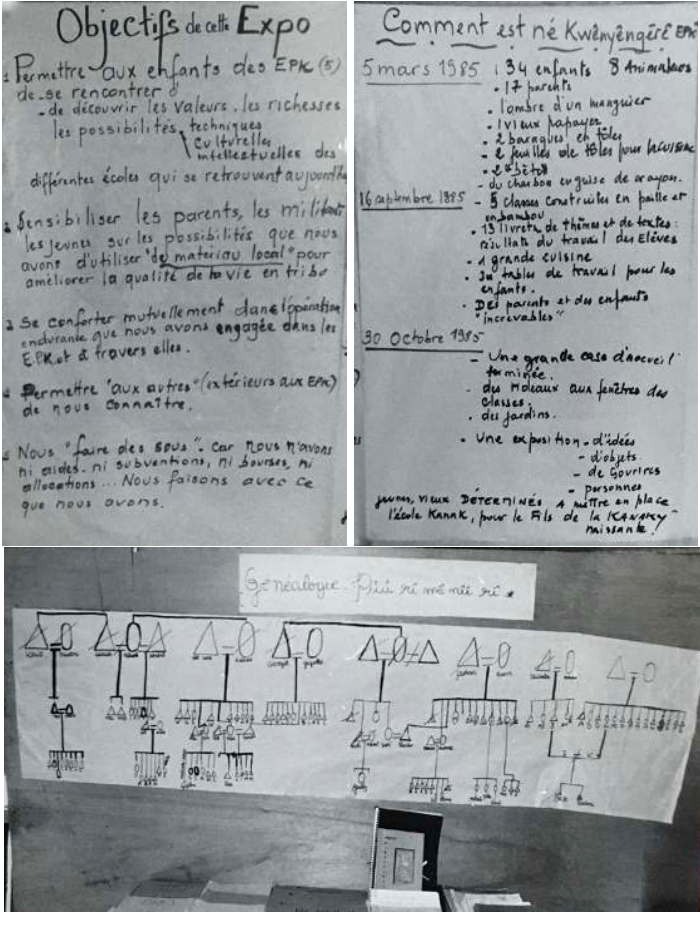

Documents 1-3. - Extraits de l'exposition de l'EpK de 30 octobre 1985 (clichés Leblic)

cèmuhî et Françoise ceux sur cinq langues du nord : wâi, jawe, caac, nyâlayu et kumac ${ }^{16}$.

"Ces stages, en fait ouverts à tous les locuteurs intéressés, ont été suivis avec une intensité et une participation active cextraordinaire volonté de réappropriation et de ce climat de grande motivation, les objectifs fixés ont pu

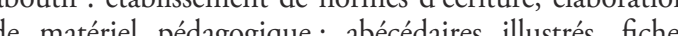
d'éveil pur déngegique: abécedaires illustrés, fiches déveil sur differents thèmes de la culture traditionnell (nomenclatures du monde naturel, savoir technologiques,

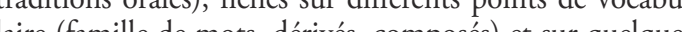
laire (famille de mots, dérives, composés) et sur quelques eléments de grammaire. Dans la dynamique creeee par ces stages, differentes initiatives ont eté prises : groupes de travall et de reflexion daniter groupes culturels (GDPL) soutenus par la région, organisave

Dans sa fiche documentaire de 1986, dans les Doints forts de ses activites, Jean-Claude souliz d'ailleurs :

"Réorientation de la recherche vers le comparatisme et

14. J'ai pu voir les magnifiques documents pédagogiques quilis avaient élaborés à la main sur la faune et la flore ainsi que des abécédaires. Malheureusement, je n’ai pas réussi à les retrouver

15. Chaque année, les chercheurs CNRS doivent remplir une fiche documentaire, en plus de leur rapport d'activité, pour rendre compte de leurs activités sur l'année écoulee. Aujourd' hui, en SHS, nous remplissons en ligne le RIBAC (Recueil d'informations pour un

16. Déàà, en 1978, elle pointait dans son rapport d'activité que « lintérêt qui se manifeste sur place pour la culture locale rend plus accessibles et utiles (projets d'enseignement des langues vernaculaires, diffusion de la littérature orale) 
Il mentionne également que

«la poursuite de ces expériences et leur élargissemen sont actuellement souhaités et un nouveau projet, soumi pour financement à la DGRST, est actuellement mis su pied sous la responsabilité d'A. Bensa. Notre objectif est de concilier travaux de recherche et tâches de formation en travaillant dans un cadre pluridisciplinaire regroupant lin-
guistes, ethnologues, historiens et économistes. » (Extrait guistes, ethnologues, historiens et
du rapport de 1985-1987 de JCR)

Dans son rapport d'activité à deux ans (juin 1985juin 1987), Jean-Claude note quil se consacre " dorénavant de façon suivie aux recherches comparatives (les langues néo-calédoniennes et l'ensemble océanien) ». Cela ne l'empêche pas de continuer "compte tenu de la situation locale " à répondre "sur place aux demandes de formation concernan "sur place aux demandes

Michel Ferlus ${ }^{17}$ fait état, dans son rapport du printemps 1991, de :

«[...] l'aspect valorisation puisque J.-C. Rivierre a mené à son terme, avec son épouse et des collègues, un programme de tisé par l'élaboration de documents pédagogiques et l'organisation de stages de formation des mâtres mélanésiens. "

Ferlus conclut sur le "cas exemplaire d'un chercheur qui, sur un terrain bien délimité, sait mene son travail jusqu'au bout avec conscience et compé tence. »

Cet investissement pour la reconnaissance des langues kanak, sans commune mesure à cette époque, fut donc pionnier. Il eut lieu quelques années avan que le mouvement indépendantiste, avec le boycott de l'école coloniale, lancé en février 1985 à Nakéty durant les événements, ne débouche sur le développement des écoles populaires kanak (EPK), lesquelles avaient pour principal objet de baser l'enseignement avaient pour principal objet de baser lenseigne.

Les Rivierre ont toujours eu à coeur de transmettr leur savoir et ont toujours participé à l'enseignement, que ce soit dans les formations DEA du LACITO, qu l'université ou à l'EHEss (notamment la FRAO) en linguistique océanienne.

Lors de la mission de 1986, Françoise a pu diffuser le lexique nyâlayu qu elle avait réalisé cette même année en collaboration avec Martine Mazaudon, à la demande des locuteurs ; l'objectif prévu était d'introduire leur langue dans l'enseignement dès l'école primaire. Cela témoigne encore de son souci de répondre à ce qu'on appellerait aujourd'hui la demande sociale . et de son souci militant de défend l'enseignement des langues kanak.

En 1998, Jean-Claude est responsable du projet CNRS-ADCK «Les langues de Nouvelle-Calédonie sur internet " qui comprend la réalisation de 9 cédéroms (langues kanak) pour le centre culturel Tijbaou jles 3 ćdéroms réaliśs en 1998 comptent 140 minut t et traduction accompagnée d'une présentation (voir annexe, Pangloss dans $\$$ archives orales). Toujours dans le but de transmettre et de restituer, Françoise s'est engagée dans ce projet d'archivage de documents sonores piloté au LACITO pour l'ouverture du centre culture Tjibaou en mai 1998. Pour sa part, elle a réalisé un cédérom sur le iaai et un sur le nemi.

Leur volonté de transmission pour l'enseignement des langues et la sauvegarde des traditions orales toujours été grande et pionnière. C'est d'ailleurs ainsi que Jean-Claude a énormément aidé Fabrice Wacalié en lui confant une grande quantité de docalié en luir confiant une grande quantité de documents inedits sur les ane gurd hui, heuretsement, des institutions locales ont repris le flambeau, que ce soit lADCK Centre culturel Tjibaou (CCT) ou lacadémie des langues (ALK). Cette volonté de diffusion a aussi été dirigée vers l'information tou public, grâce à plusieurs participations, tant dan le domaine linguistique que sur la situation de la Nouvelle-Calédonie. D'ailleurs, dans sa fiche documentaire de l'année 1985, au point «Diffusion de l'information scientifique et technique ", en plus des traditionnelles Expolangue du LACITO (voir https://lacito.cnrs.fr/expos/index.htm), Jean-Claude Rivierre signale des articles et in sign évén dien avec ces evén ens Le Monde diplomatique, Les Temps modernes, France de criber (media tess-monde intitulé Centre de documentation et d'échanges pour la solidarité internationale), etc. Cette valorisation des connaissances se poursuivit en 1986 ( $c f$. fiche documentaire de 1986) avec des consultations et animations de sé minaires pour l'IRFED (Institut éducation, culture et développement), l'inODEP (Institut œcuménique au service du développement des peuples) et l'AKADEK. Comme nombre d'entre nous, Jean-Claude participa également à l'exposition sur la culture kanak inaugurée à la Maison internationale de Rennes (4-11 avil 1987). Je n'en ai pas trouvé trace dans ses rapport ses rapports comme nous tous - y avait également largement participé

\section{Une reconnaissance internationale}

Jean-Claude et Françoise ont collaboré avec de nombreux chercheurs étrangers spécialistes des langues océaniennes et ils ont souvent participé à des colloques ${ }^{19}$ en Australie, en Nouvelle-Zélande, Hawai'i, au Japon, etc. Je ne pourrais pas citer ici

17. Michel Ferlus (1935-), émérite au CNRS, a travaillé sur les langues de l'Asie du Sud-Est et est spécialisé en phonologie historique. 18. Emmanuel Tjibaou, fils de Jean-Marie Tjibaou et actuel directeur du CCT, a d'ailleurs été élève de Françoise Ozanne-Rivierre qui l'a aidé pour son mémoire de maîtrise à l'INALCO sur l'expression des relations sociales dans les récits traditionnels (fwâi, nemi) de 19. Ils participèrent régulièrement aux colloques International Conference on Austronesian Languages (ICAL3 3 à Bali en 1981
ICAL à Honolulu en 1991 ) dont la $11^{\circ}$ conférence fut d'ailleurs co-organisée par le LACrTo et le CRLA à Aussois en juin 2009 et aux Conferences on Oceanian Linguistics (cool3 à Hamilton en Nouvelle-Zélande en 1997, cooL 5 à Canberra en 2002), etc.

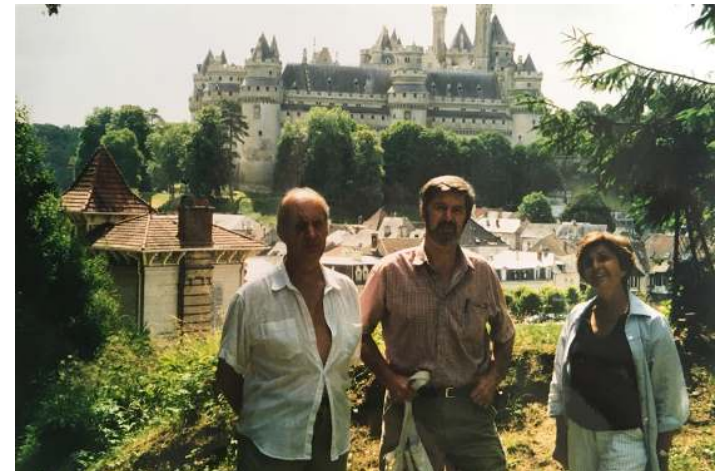

Рното 13 - Françoise, Jean-Claude et Andrew Pawley devant le château de Pierrefonds (juin 2001) (cliché Pawley)

linguiste et femme

Conference on Ocea-

Zealand), en 1997

avec Andrew Pawley
Рното 14. - Françoise

et Medina Pawley,

Hollyman, chez Jim

nic Linguistics de

l'University of Wai-

kato (Hamilton, New

(cliché Pawley). Voi

aussi photo 1 p. 223

i it.

6

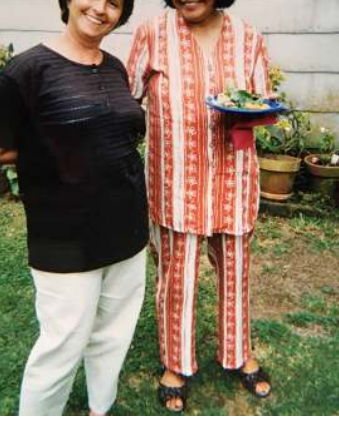

tous les collègues avec lesquels ils ont collaboré et la présentation qui suit n'est pas exhaustive.

Tous deux ont contribué à l'hommage qui a été consacré à George W. Grace (1921-2015), professeur de linguistique à l'Université d'Hawai'i, spéci lisé dans la linguistique historique et comparative, les langues austronésiennes et, tout particulièrement les langues mélanésiennes (FOR, 1991a ; JCR 1991a). Haudricourt note à ce propos, dans son rapport de direction de recherche de 1975, que :

" la découverte et la description de ces langues [à tons du Pacifique] est son œuvre (en 1955, le prof. Grace, actuellement à Honolulu, s'était aperçu qu'il y avait des tons mais, devant la difficulté d'observation et de description, n'en parlé que dans sa correspondance privée).

Kenneth James dit Jim Hollyman (1922-2004) ${ }^{20}$ professeur à l'université d'Auckland (Nouvelle-Zé lande) de 1954 à 1986 (date de sa retraite) où il enseigna le français de 1966 à 1986, était un collègue avec qui nous avons tous beaucoup échangé et par-

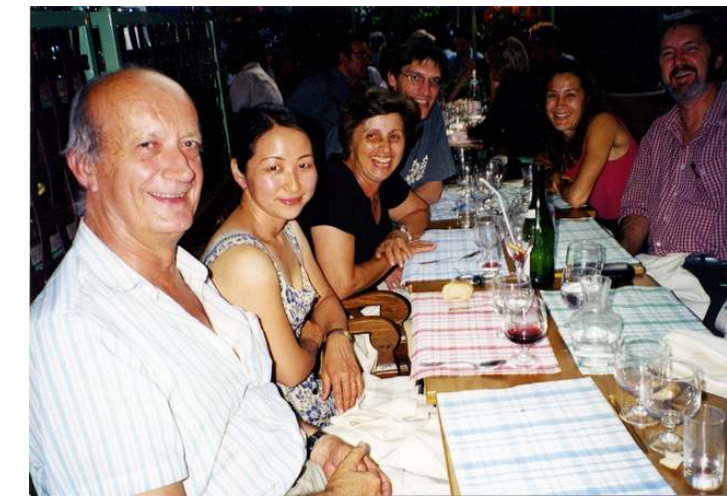

Pното 15. - Dîner à Paris, en juin 2001, à l'occasion de la visite d'Andrew Pawley au LACITO : Andrew à côté d' 'sabelle

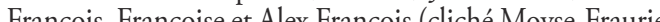

tagé de bons moments (livre commun avec FOR en 1987 et participation à son hommage 1986).

Robert A. Blust (1940-) est professeur de linguistique à l'Université d'Hawai'i à Mānoa, spécialist des langues austronésiennes, de linguistique histoutilisé les travaux. Il coordonna notamment l'ouvrage d'hommage à Grace auquel le couple Rivierre participé (FOR, 1991a ; JCR, 1991a).

Andrew Pawley (1941-) est un linguiste australien, professeur à l'ANU à Canberra, spécialiste reconnu l'international des langues austronésiennes et papoues. Il a proposé, en 1966, une classification des pous. II a propese, en 1966, undrew Pawley, 1996). C'c etait un bon collegue des Rivierre et, comme bo nombre de leurs collègues et amis, il a passé aussi agreables moments à Retheuil en 2001 suite à un invitation qui lui avait été faite au LACITO (voir photos 13-15). C'est donc tout naturellement qu'il a proposé ici une contribution.

Darrell Tryon (1942-2013), linguiste néo-zélandais, professeur à l'ANU, spécialiste des langues austronesiennes, du Vanuatu et des Salomon comme des pidgins et créoles d'Océanie, a rencontré les Rivierre à Rivierre ont participé à plusieurs de ses publications, sur les changements linguistiques (FOR 1994b; JCR, 1994b) ou au fam Comprative Austronesic JC ustronesian DicMalcom Ross (1942-) 1995).

Malcom Ross (1942-) est un autre linguiste ausalien professeur à l'ANU à Canberra, spécialiste des tique historique et des contacts de langue, avec leque ils ont beaucoup échangé (voir ci-dessous p. 139). rique et de lexicographie, dont Françoise a beaucoup de nombreuses reprises, dont certaines au LACITO. Les angues austronésiennes et papoues, de la linguis-

20. En tant que militaire, il alla sans doute pour la première fois en Nouvelle-Calédonie à lầge de 21 ans, en avril 1943, durant la Deuxième Guerre mondiale. J'ai eu la chance de me rendre par deux fois chez lui en Nouvelle-Zélande. Lui et sa femme Jeannette me reçurent a chaque fois une semaine, en mai 1985 alors que jetais de passage pour me rendre en Nouvelle-Calédonie (arrivée Auckland le lundi et envol pour Nouméa le samedi) et, de meme au retour en mai 1986, mais de façon plus rapide. La deuxième fois

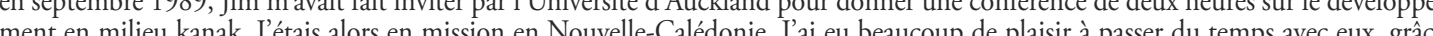
ment en milicu kanak. Jetais alors en mission en Nouvelle-Caledonic. Jai eu beaucoup de plaisiri a passer du temps avec eux, grâa 21. La différenciation du proto-polynésien en deux groupes (langues tongiques et langues polynésiennes nucléaires), d'après les travaux de Pawley en 1966 (voir carte enregistrée) (https://fr.wikipedia.org/wiki/Andrew_Pawley). 

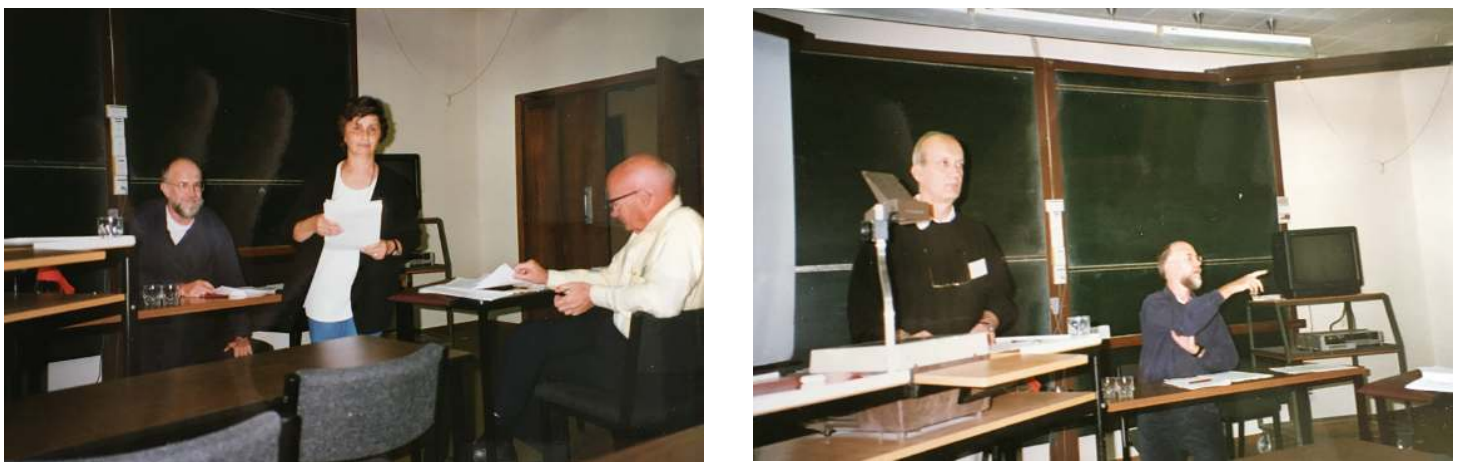

Рнотоs 16-17. - Françoise et Jean-Claude lors de la $3^{\mathrm{e}}$ conférence internationale sur la linguistique océanienne (TRICOLCool3), à Hamilton (New Zealand, 15-17/01/1997). En face de Françoise est assis Joseph Finney (clichés Pawley)

John Lynch (1946-), linguiste australien ayant fait la plus grande partie de sa carrière au Vanuatu, y a été professeur et directeur de la Pacific Languages Unit de l'Université du South Pacific à Port-Vila. Il a aussi travaillé pour l'Université de Papouasie Nouvelle-Guinée. Il était venu passer six mois en France au cours du second semestre 2000 au LACITO-CNRS Villejuif, afin de faire des recherches sur les liens historiques entre les langues de la Nouvelle-Calédoni et celles du sud du Vanuatu. Il avait alors travaille avec les Rivierre ; leur collaboration fut fructueuse (voir Lynch \& Ozanne-Rivierre, 2001) et permi d'approfondir les études de ces interrelations. Joh Lynch, comme de nombrux collaborans. Joh Ryiviere, garde un excellent souvenir de ceurci, qui excili, qui étaient devenus des amis et auxquels il dédie so article présent dans ce volume.

Meredith Osmond ${ }^{22}$ est une professeure émérite de linguistique à l'ANU, spécialiste notamment de linguistique historique et du proto-océanien. Les $\mathrm{Ri}$ vierre ont fait de nombreux colloques avec elle (voir dans ce volume photo 1 p. 223).

Gunter Senft (1952-), linguiste allemand, professeur au Max Planck Institute de Nijmegen (Pays-Bas), spécialiste des langues austronésiennes, notamment des îles Trobriand, est un collìgue avec lequel Françoise a publié sur la deixis spatiale (1997b, 2004a).

publié sur la deixis spatiale (1997b, 2004a).
Paul Geraghty est professeur associé à l'University Paul Geraghty est professeur associe à l'University
of the South Pacific, School of Language, Arts and of the South Pacific, School of Language, Arts and
Media. Il a écrit de nombreux articles sur les langues Media. Il a écrit de nombreux articles sur les langues l'histoire. Jean-Claude et Françoise l'ont côtoyé lor de colloques.

Citons encore rapidement: Even Hovdhaugen (1941-2018), linguiste norvégien, professeur l'université d'Oslo et spécialiste des langues polynésiennes: Ross Clark, de l'université d'Auckland Midori Osumi, linguiste japonise ayant travallé notamment sur le tîrî̀ de Nouvelle-Calédonie (aire xârâcùù), affiliée à la Tokyo Woman's Christian Uni-

versity, avec laquelle ils ont participé à un colloque à Tokyo en 2000 ; Chris Ballard, Senior Fellow, School of Culture, History \& Language, College of Asia $\&$ the Pacific, ANU, qui est venu au LACITO et avec lequel nous avons tous eu divers échanges.

En 1989, Françoise et Jean-Claude furent également visiting scholars durant presque trois mois à Berkeley aux USA ; le but de ce séjour était :

« [la prise de] contacts avec l'équipe travaillant au procted Thesaurus) où deux chercheurs de notre département
sont engagés. Leur méthodologie et leurs outils de travail sont engagés. Leur méthodologie et leurs outils de traval pourront inspirer un projet parallele sur les langues oce
niennes » (Extrait du rapport de 1987-1989 de FOR)

Très modestes, ils ont toujours été peu friands des honneurs, ce quavait noté à l'époque Alexis Rygaloff ${ }^{23}$ dans son rapport au CNRS :

"M. Rivierre s'impose désormais, non seulement en France, mais sur le plan mondial, comme le meilleur spé cialiste des langues a tons du Pacifique. Sa promion a rapport d'Alexis Rygaloff, mai 1975)

Mais, comme noté quatre années plus tard par son rapporteur au CNRS, Gabriel Manessy ${ }^{24}$, cette modestie ne les empêchait pas d avoir une grande notoriété dans le milieu de la linguistique océaniste :

«Il s'agit d'un chercheur fort actif et de notoriété internationale dont le renouvellement ne saurait être mis en question. (Extrait du rapport de Gabriel Manessy, mai 1979)

Ce qui fut confirmé bien des années plus tard en 1992 :

«Si l'on consulte les rapports qui, depuis des années ont jalonné le parcours scientifique de M. J.-C. Rivierre (y compris le dernier, celui de Emilio Bonvini ${ }^{25}$ [d'automn 1991], " on voit toujours revenir des termes comme "excellence", "régularite",", "solidité". J'ajouterai pour ma part tout en partageant ces avis - que notre collègue est une domaine de la linguistique de terrain : sa pensée est péné-

22. Autre point de rencontre avec Françoise, elle a publié en 2009 un article sur les jeux de ficelle comme un champ décevant pour la reconstruction lexicale (Osmond, 2009).

23. Alexis Rygaloff (1922-) est linguiste et sinologue, aujourd' hui rattaché au centre d'études coréennes de Paris Diderot, INALCO, EHESS

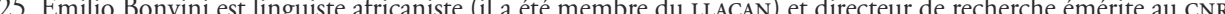

trante et son parcours rigoureux. Je donne un avis très favorable à sa promotion en DR1. " (Extrait du rapport de Blanche-Noëlle Grunige ${ }^{26}$ de 1992 sur JCR)

La reconnaissance internationale dont ils bénéficient de la part de leurs collègues est visible dans le dossier proposé ici, au travers des contributions John Lynch, Andrew Pawley, Christine Jourdan et Marie Walworth, la dernière jeune chercheure à être venue au LACITO travailler avec Jean-Claude.

Je vais maintenant présenter les carrières de Françoise Ozanne-Rivierre et Jean-Claude Rivierre. En ce tempslà, les dossiers des personnels temporaires du CNRs ${ }^{27}$ étaient examinés chaque année par les commissions concernées, ici, celle de linguistique générale ${ }^{28}$.

\section{Françoise Ozanne-Rivierre, de l'ethnologie à la} linguistique

Françoise Ozanne-Rivierre (2/01/1941-11/11/2007) de deux ans et demi la cadette de son mari, aurai commencé par se former à l'ethnologie avec Jean Guiart, lequel avait confié à Alain Saussol (2018: 611) que c'était l'une de ses meilleures étudiantes. 611) que c'était l'une de ses meilleures étudiantes
Puis, sans doute au retour de son premier séjour ${ }^{29}$ Puis, sans doute au retour de son premier séjour ${ }^{29}$
en Nouvelle-Calédonie où elle avait accompagné son en Nouvelle-Calédonie où elle avait accompagné son
mari Jean-Claude, après avoir obtenu en 1970 un mari Jean-Claude, après avoir obtenu en 1970 un diplôme d'études approfondies en sciences sociales $\left(\right.$ EPRASS $^{30}$ ) à l'École pratique des hautes études (EPHE) $6^{e}$ section, elle s'est tournée définitivement vers la linguistique et soutint son doctorat en linguistique en 1973 sur le iaai, langue mélanésienne d'Ouvéa (Nouvelle-Calédonie), à l'Université de Paris 3 (publié en 1976), juste après avoir intégré le CNRS comme attachée de recherche en 1972 dans le programme d'ethnohistoire du Pacifique ${ }^{31}$ sous la direction d'André-Georges Haudricourt. Chargée de recherche, elle devint membre du LACITO à sa création en 1976 devint membre du LACITO à sa création en 1976
jusquà sa retraite en 2006. Auparavant, comme

26. Blanche-Noëlle Grunig (1939-2013), spécialiste de germa

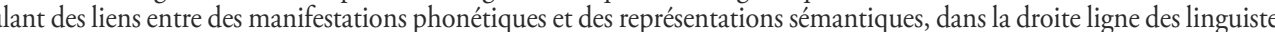
tels que Chomsky. Elle fit une grande partie de sa carrière de professeure à Paris 8 .

27. Ce n'est qu'en 1982, par la loi du 15 juillet, dite loi Chevènement, que les personnels chercheurs comme ingénieurs, techniciens et administratiff (ITA) passent sous le régime de la fonction publique, ce qui n'a pas
CNRS, car ils avaient le choix de rester dans l'ancien statut ou de passer fonctionnaire.

28. Qui fut successivement, pour la période qui nous concerne, la $22^{\circ}$ : Linguistique générale, langues modernes, littérature conparée (1959-1966), la $28^{e}$ : Linguistique génerale, langues et littératures étrangères $(1967-1970)$ devenue $30^{\circ}(1971-75)$, puis $35^{\circ}$ (1976-82) sous le mème intitule, puis la section 42 Sciences du langage (1983-91), pour être depuis 1992 et jusqua aujourd hui la 34 d'abord Représentations, langages - Communication jusque 2004 puis Langues, langage, discours de 204 a 2012). Aujourd' hu

29. Après le décès de Françoise, Jean-Claude me confia des cahiers de notes sur les clans du sud (puisque j'y avais fait des enquêtes anthropologiques lors de mes premiers terrains en pays kanak), preuve de l'intérêt premier de FoR pour l'ethnologie kanak comme nous le verrons aussi à propos des jeux de ficelle dans ce même volume.

30. Elle avait commencé à se former à l'EPRASS «à la linguistique de terrain ou de langues exotiques comme on disait alors $[\ldots .$. enseignement initié par Jacqueline Thomas et Luc Bouquiaux » (comm. pers. de Paulette Roulon-Doko, 20/10/19).

31. Il s’agit de la Recherche coopérative sur programme RCP 259, équipe pluridisciplinaire dédiée à lethnohistoire du Pacifique, dirigée d'abord par Jean Guiart, puis par André-Georges Haudricourt entre 1965 et 1974. D'après Jean-Claude Rivierre lui-même (https://archivesetmanuscrits.bnf.fr/ark:/ $12148 /$ ccl $02782 \mathrm{~h}$ ), les linguistes de cette RCP furent rattachés au LACITO dès sa création en 1976 où ils on constitué un département oćániste. Ils ont tous euvré successivement à l'etude synchronique et diachronique des langues parlées dan et pluridisciplinaire des langues ayant été privilégiée au départ au sein du LACITO, «les chercheurs océanistes ont mis à profit leur présence sur le terrain pour recueilliri des textes oraux formalisés oun non en contrepoint de leurs études descriptives. Ce recueil de documents sonores avait pour ambition de constituer un corpus ill ustratif des langues et des cultures, mais aussi de contribuer à leur sauvegarde ". 
La nature du travail qu'elle prenait en charge consistait à mettre en fiche et dactylographier les documents sur les langues des Nouvelles-Hébrides recueillis par Jean-Claude et avant lui pour la RCP 27
de Jean Guiart créée en 1963 et consacrée à l'Océanie (voir Gaillard, 2004 : 299). Il est noté qu'elle est "particulièrement apte au poste ». Sur son rapport de titularisation (fin de stage de six mois), Haudricourt note que :

"Madame Rivierre est tout à fait qualifiée pour le travail assez fastidieux qui lui est demandé et j'espère qu'elle pourra continuer ce travail jusqu’à la mise au net pour publication."

D'où la proposition d'engagement définitif qu'il fait en tant que chef de service, le 17/10/1967, avant le terme de son stage. Françoise fut donc engagée le 4/07/1967; sur son dossier (au 18/5/67) figure une partie de son cursus universitaire, avec notammen une licence de sociologie.

Lappréciation faite chaque année par Haudricour pointe un " travail efficace et intelligent » avec une notation totale de 18/20 (capacités techniques: 5 , ponctualité : 4, efficacité : 5 et soin dans l'exécution : 4). En 1969, Haudricourt, qui est toujours son che de service, précise la «nature du travail effectué pa l'agent » et qui porte dorénavant sur le iaai :

"Mise en fiche des textes recueillis par J. Guiart en $1950^{32}$ en langue mélanésienne d'Ouvéa (Loyalty), éta bissenent de la traduction du texte et du fichicr, mélanésien-français; verification auprès d un informateu vivement à son travil et le poursuit avec compétence, diligence et ponctualité. Travail intelligent et approfondi. (AGH, EPHE, Paris, le 5/11/1969)

Jusqu'en 1971, Françoise resta «enquêteur adjoin qualifié » catégorie 2B $4^{e}$ échelon, travaillant pour RCP 259 sous la direction d'A.-G. Haudricourt. Elle fut intégrée dans le corps des chercheurs le $1^{\text {er }}$ janvier 1972 sur proposition de la commission 28 de linguistique générale.

Attachée puis chargée de recherche en linguistique : $d u$ iaai aux langues de Hienghène... description et typologie

Après avoir effectué son premier terrain de recherche en solo à Ouvéa de juillet à octobre 1971 pour réaliser sa thèse de $3^{\text {e }}$ cycle, elle devint attachée de recherche. En 1977, elle passa chargée de recherche de première classe (CR1).

Dans son rapport d'activité de 1977, Françoise note, à propos de la littérature orale, que :

"Tous ces textes, outre leur intérêt linguistique, ap-
Mélanésiens de Nouvelle-Calédonie des renseignements "une grande richesse. Mais pour urgent et passionnan u'il soit, le sauvetage de cette littérature orale est une âche accaparante et le temps passé à fignoler mot à mot, ation soignée, finit par entraîner le linguiste un peu plus oin quil ne le voudrait de ses préoccupations strictement linguistiques. C'est pourquoi j'ai décidé, une fois les textes nemi publiés, de ne préparer qu’à temps réduit la même opération pour les textes fwâi, jawe et iaai, pour m'attache en priorité à la mise au point des dictionnaires monographiques, des lexiques comparés et à une réflexion, en collaboration avec les autres linguistes de la même région, sur

En 1985, elle candidata au concours DR2 en section de linguistique 42 intitulée Sciences du langage 1983-1991) ; D. François ${ }^{34}$, qui rapporta sur son dossier, mentionna que :

"aux recherches proprement linguistiques (centrées ntanne détermination) s'ajoute, dans le cadre des langues à tradition orale, un travail ethnolinguistique. [ . Dans cadre de l'atp sur l'expression de l'espace dans le domaine uustronésien, Mme Ozanne-Rivierre a exploré plusieur angues océaniennes. Bref, la linguistique et l'ethnolinquistique s'imbriquent étroitement dans son travail. Paraguistique s imbriquent etroitement dans son travail. ParalRivierre a mené des études appliquées à l'enseignement dans les langues vernaculaires. [...] Le projet de recherch que présente Mme Ozanne-Rivierre est très cohérent : porte essentiellement sur la phonologie comparée et diachronique dans le nord de la Grande Terre et vise l'établissement de listes de correspondances sur lesquelles seraien fondées des conclusions sur la chronologie des changele proto-système nord calédonien. Une étude comparative en syntaxe est également envisagée. [...] Le travail de Mme Rivierre apparait comme très méthodique, cohéren et soucieux d'applications. Nous donnons un avis trè favorable pour son accès au titre de DR2."

Dans son rapport d'activité à deux ans (juin 1987juin 1989), Françoise écrit que :

"Ces deux années ont surtout été consacrées à l'élaboration de synthèses comparatives tant dans le domaine phonologique que syntaxique sur l'ensemble des langues de la Nouvelle-Caledonie et des Loyauté. Mes recherches linguistique dans les langues de cette région et j’étudie tuellement les conséquences d'un contact continu sur l'île d'Ouvéa entre une langue mélanésienne, le iaai, et un outlier polynésien, le fagauvea, établi sur cette île depuis quelques siècles. " (Extrait du rapport 1987-1989 de FOR, voir colloque Canberra 1989 et publication avec Hollyman, 1987)

Elle précise également la part de son travail dans cette synthèse comparative commune avec JCR sur : traduction, notes explicatives pour en donner une présendes problèmes grammaticaux spécifiques à ces langues. » ments linguistiques, sur la classification des langues et sur

32. Dans l'avant-propos du dictionnaire de iaiai (1984 : 9), Françoise écrit que ces textes avaient été recueillis par Jean Guiart en

33. Françoise note, dans l'introduction de ce dictionnaire (Ozanne-Rivierre, 1984 : 11), que « Son point de départ fut un ensemble de textes recueillis à Ouvéa en 1948 par Jean Guiart et transcrits par ses informateurs eux-mêmes dans Le lexiquegraphe iaai utilisée dans la Bible.

34. Denise François (1934-1993) fut notamment professeure de linguistique générale et appliquée à'Université Paris 5. "les différents processus qui ont permis le développement des voyelles nasales phonologiques dans les langues de Nouvelle-Calédonie et les mutations consonantiques qui en ont résulté dans certaines langues [...]. Ma partie concernait plus précisément les langues du Nord où les consonnes finales sont conservées et où les voyelles nasales se sont surtout développées par assimilation progressive
$[\ldots]$, (Extrait du rapport 1987-1989 de FoR, voir aussi com. à Aukland en janvier 1988)

Elle présente ensuite l'étude comparée, dans le domaine syntaxique, des types formels de détermination progressive dans les langues kanak (voir table ronde LACITO 1988) :

«Trois types, diversement représentés selon les langues, sont attestés : détermination directe, détermination ind de type appositionnel où la chose possédée is peut êt mise en relation avec un possessur (nécessie neut être mé) qu'à l'aide d'un 'classificateur' seul apte à recevoir détermination possessive (ex barque/bateu du chef, barque du chef'). Ce type appositionnel, marginal dans langues de Nouvelle-Calédonie, est très dévelopé é Ouvéa [. . " (Extrait du rapport 1987-1989 de por)

En 1990, dans sa fiche documentaire, François stipule que ses thèmes de recherche sont la phonologie diachronique (dans le domaine océanien) avec l'étude du changement linguistique et les problèmes de reconstruction. Dans le rapport à quatre ans (1987-1991), elle rajoute la lexicor (1987-199u) ele Donse le rapporta à quec lelade Balade, nord de la Grande Terre). En matière de phonologie diachronique, elle mentionne les deux aspects qu' elle a étudiés :

aspects qu elle a étudiés : ne sont pas seulement riches en modèles de changements phonologiques qui intéressent la linguistique générale, elles témoignent aussi, par rapport à leur ancêtre protoocéanien, de traits conservateurs qui éclairent certains problèmes de reconstruction propres à ce groupe linguistique. " (Extrait du rapport 1987-1991 de FOR)

Dans les changements internes, elle note le développement de voyelles nasales phonologiques et les mutations consonantiques qui en ont résulté (colloques Lille 1987 et Aukland 1988 ; publi. 1989) et les consonnes finales non etymologiques, trace fossile d'anciens relateurs (publi. 1991 en hommage à G.W. Grace). En matière de reconstruction, communication a Honolulu en 1991 a mis en relief certains traits conservateurs des langues kanak par rapport au proto-océanien et le fait que :

«[ce] témoignage des langues de Nouvelle-Calédonie remet en cause la nouvelle organisation du systeme consonantique proto-océanien proposée par M. Ross (1989) souvent prise en compte depuis lors par les comparatistes océanistes. » (Extrait du rapport 1987-1991 de FOR)

Pour la période juin 1987-juin 1991, Françoise souligne que, ces quatre dernières années, ses re-

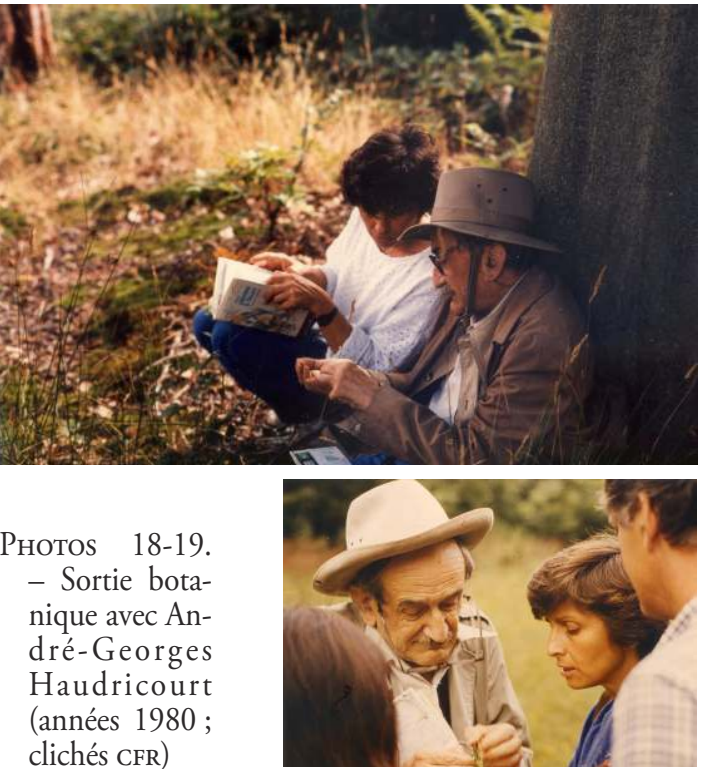

clichés CFR)

cherches ont donc été axées sur la phonologie diachronique (étude des changements internes attestés dans les langues de Nouvelle-Calédonie et problèmes de reconstruction), les contacts de langues et le changement linguistique, des synthèses comparatives sur les structures de détermination possessive et l'expression de l'espace et la lexicographie (dictionnaire nyêlâyu-français de Balade, nord de la Grande Terre) Elle a étendu le travail comparatif déjà réalisé sur les langues du Nord aux langues des îles Loyauté (voir tro 1986). En 1988, elle insiste sur la phono gie suprasegmentale et la phonologie diachronique. De juin 1991 à juin 1995, ses principaux axes de recherche sont la linguistique historique et comparative, dans la lignée des travaux d'André-Georges Haudricourt, avec une approche structurale qui prend donc en compte les causes internes et externe déterminant les changements, et la phonologie diachronique, les études typologiques dans l'aire océanienne et l'étude descriptive du nyêlâyu.

Grâce à ses fiches documentaires annuelles, on sait quelle mène ses recherches jusqu'en 1995 au sein département Océanie - Langues et cultures du Pacifique dont elle ét responshle au LACTO depuis 199235 . Ensuite, à partir de 1996, suite à l copu nisation du LACTO è à la suppression des dépro mation des départements "géographiques " - le département Océani étant le dernier (voir Leblic et Masquelier, 2020) elle rejoint l'équipe Typologie et changements linguistiques (TCL) où ses recherches portaient sur la linguistique historique et comparative.

En 1988, ses thèmes de recherche étaitent la phonologie suprasegmentale et la phonologie diachrohique. Pour 1989-1995, ses travaux ont concerne la phonolo dian . proto-calédonien, les études typologiques dans l'air

35. Auparavant c'ćtait Jean-Claude Rivierre qui en était le responsable, fonction quil a quittée qund il est devenu directeur du LACITO en 1992. 
tive océanienne (famille austronésienne et, plus particulièrement, le groupe océanien) ainsi que les études descriptives en Nouvelle-Calédonie, dont celle du nyêlâyu. En 1993-1994, signe de l'intérêt qu'elle toujours eu à cour de transmettre et de former de futurs chercheurs (que l'on voit aussi dans son enselgnement régulier dans les séances de DEA du LACITo), elle note le tuteurage de la thèse d'Isabelle Bril inscrite avec A. Culioli à l'Université Paris 7 sur la langue nêlêmwâ (Nouvelle-Calédonie). Elle fut d'aileurs membre du jury de soutenance (16/01/1995)

Malgré ses soucis de santé, Françoise continua à se former, notamment à l'utilisation de la base de données MARIAMA ${ }^{36}$, conçue par R. Nicolai ${ }^{37}$ et J. Zer$n r^{38}$, en participant au stage à Marseille-Luminy sur "Gestionnaire d'hypothèses et d'analyses de données" organisé du 10 au 18 juin 1996. Ce logicie de dialectologie comparative devait aboutir, à terme

« [à] la constitution [d'une] base de données [devant] permettre la reconstruction de terminologies thématiques en proto-calédonien mais aussi dans les trois sous-groupes déjà définis sur des critères phonologiques (Loyauté, Nord et Sud de la Grande Terre). » (Extrait du rapport 19951997 de FOR)

Phonologie comparative, linguistique historique et reconstruction, deixis spatiale.

Si l'on récapitule rapidement ses différents thèmes de recherches notés sur les fiches documentaires annuelles de 1988 à 2002, on trouve :

- la phonologie suprasegmentale et la phonologie diachronique (1988)

- a linguistique comparative océanienne (1989);

- la phonologie diachronique (dans le domain océanien) : étude du changement linguistique et problèmes de reconstruction (1990)

- la linguistique historique et comparative (famille austronésienne et, plus particulièrement, dans le groupe océanien) et reconstruction du proto-calédonien; le changement linguistique et le problème de la reconstruction dans le domaine océanien ; la description des langues de NouvelleCalédonie ; la phonologie diachronique ; les études typologiques dans l'aire océanienne et l'étude destypologiques dans l'aire océanienne et l'étude des-
criptive du nyelâyu de Nouvelle-Calédonie (19901996);
- la linguistique océanienne : phonologie diachronique et la reconstruction lexicologique néo-calédonienne (1996-99) auxquelles elle ajoute les études typologiques (négation, deixis spatiale) en 1999-2000 - aux thèmes précédents, elle ajoute en 2000-200 a préparation d'un ouvrage de synthèse sur les modèles de changements attestés dans le groupe des langues néo-calédoniennes en collaboration avec JCR et dans les études typologiques, en plus de l'expression linguistique de l'espace (FOR, 2004a), l'évolution de constructions verbales sérielles (FOR, 2004b ; FOR et JCR, 2004a-b-c) ;

- la phonologie diachronique et la reconstruction en linguistique océanienne : évolution du système des pronoms personnels en écenien oriental (avec Jes pronoms person' d'uns ecéanien oriental (avec John Lynch), étude d un changement récurrent ( $\mathrm{t}>\mathrm{k}$ ) dans la famille austronésienne et l'évolution des formes canoniques dans les groupes des langues néocalédoniennes (avec JCR); ; études typologiques : l'expression linguistique de l'espace, les construction verbales sérielles (cvs) (voir Bril et FOR éds, 2004). Côté enseignement ${ }^{39}$, elle a participé à toutes les sessions d'enseignement en DEA du LACITO. En 1993 1995, elle fut chargée de cours avec Jean-Claude l'eHess pour un séminaire bi-mensuel de "linguistique océanienne " (24 heures annuelles) devenu en 1995-1996 « Linguistique et ethnolinguistique océ niennes En 1999-2000, elle fut chargé de cours ni l'Unice cours pour la licence Sciences du langage (sL340) où elle enseigna la linguistique océanienne avec un cours sur «le iaai, langue de Nouvelle-Calédonie " $(12 \mathrm{~h})$. Elle et son mari partici pèrent aussi régulièrement aux enseignements EHESS de la Formation à la recherche anthropologique sur 'Océanie (frAO).

Du côté de la «valorisation ", il faut citer le cédérom de quinze textes nemi édité pour le CCT de Nouméa en 1998. En plus de ces quinze textes, on en trouve un sur « l'origine de la monnaie traditionnelle kanak » en langue pije. En 1999, elle participera a contrat d'édition du cédérom signé entre le LAcITo

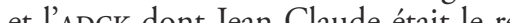
1999-2000, elle responsable. En 19 traditions orales dans l'aire culturelle iaai pour médiathèque du ССт (avec Claire Moyse-Faurie). Comme nombre d'entre nous, elle fut consul-
tante pour la mission de préfiguration du musée de
36. "Le logiciel mariama.1, "gestionnaire d'hypothèses et de bases de données", avait été conçu au début des années 1990, puis développé ultérieurement avec la collaboration de Jeanne Zerner. Son objectifétait, d'une part, de fournir un environnement de travail

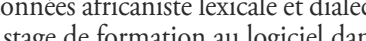
tologique $[. .$.$] La documentation du logiciel MARIAMA.1 a été réalisée pour servir de support a un stage de formation au logiciel dan$
le cadre d'une École d'été du CNRS tenue à Luminy (1996) pour des chercheurs issus de diverses institutions de recherche francaises et européennes. La programmation du logiciel a été stoppée en 2001 par manque de financement et de ressources humaines dédiées. Cette documentation nest donc plus actuelle mais elle a l'intérêt de fournir une information sur un moment de la recherche dans le domaine des bases de données linguistiques et sur la "philosophie" de ce logiciel développé entre 1994 et 2001. » (https://bcl.cnrs.fr

37. "Robert Nicolä est un chercheur spécialiste des langues nilo-sahariennes, ancien professeur de l'Université de Nice. Il a, par le passé, dirigé un groupe de recherche européen (GDRE Diffusion lexicale en zone sahelo-saharienne) dans le cadre duquel nous avion

38. Jeanne Zerner a été ingénieure en développement d'application au LLACAN CNRS de 1995 à 2006. Elle est aujourd' hui directrice 39. Cette liste n’est bien sûr pas exhaustive.
l'Homme, des arts et des civilisations (MHAC), au sein du groupe " Océanie ».

Principales contributions théoriques de Françoise

Au fil du temps, cette ancienne disciple d'AndréGeorges Haudricourt et de Claude Hagège, est devenue une spécialiste internationalement reconnu pour sa contribution au comparatisme des langues océaniennes (FOR, 1986; Moyse et FOR, 1983a-b). Elle l'est aussi pour les descriptions qu'elle a publiées de plusieurs langues (fagauvea, fwâi, iaai, jawe, nemi nyelâyu, pije) de Nouvelle-Calédonie, aire linguistique réputée pour son extrême diversité et sa grande complexité puisqu'elle ne comptepas mé exan complexité pusque con huit langues réparties en trois sous-groupes différents (FOR, 1975a-b, 1976a, 1984, 1991a-b ; FOR et Tein, 1975, 1979; FOR et al., 1998; FOR et Haudricour, 1982 ; FOR et Mazaudon, 1986; Hollyman et FOR,
1987 ) sans oublier la tradition orale (FOR, 1975a, 1986 ; JCR et al., 1980), etc.

Ses principales contributions théoriques porten sur la phonologie historique (FOR, 1982, 1994bc), le classement génétique des langues kanak et la reconstruction du proto-néo-calédonien (Moyse et FOR, 1983a-b, 1999 ; FOR, 1986, 1989, 1992, 2000a ; FOR et JCR, 1989). Avec André-Georges Haudricourt, puis avec Jean-Claude Rivierre, elle a permis de mettre en évidence l'appartenance de ces langues de mettre en évidence lappartenance de ces langues au sous-groupe océanien de la grande famille austro-
nésienne. À cela, il faut ajouter ses recherches sur la nésienne. À cela, il faut ajouter ses recherches sur la deixis spatiale pour lesquelles elle a apporté des données tout à fait originales (FOR, 1

ou sur les prédic 2004b).

À partir des années 1960, Françoise Ozanne-Rivierre a mené des enquêtes de terrain sur plusieurs de de documents qui ont été la source et la matière de réflexion toute sa vie durant.

Ainsi, après sa thèse consacrée à une description Ainsi, après sa thèse consacree à une description
phonologique et syntaxique du iaai d'Ouvéa (îles phonologique et syntaxique du iaai d'Ouvéa (îles
Loyauté) en 1976, elle s'est intéressée aux langues de Loyauté) en 1976, elle s'est intéressée aux langues de la région de Hienghène, publiant en 1975-79, avec la collaboration de Poindi Tein, deux volumes de textes nemi, suivis, en 1982, de l'imposant Dictionnaire thématique des langues de la région de Hienghène rédige en collaboration avec Andre-Georges Haudricourt. Elle a poursuivi ce précieux travail de description de langues, en publiant en 1998 le Dictionnaire du mpelayu de Balade à l'extêne nord de la Grande Te layn de Balade, à lextene no en collaboration ác Baptiste Boiguivie, Scholastique Boiguivie et Éliane Dedane. Enfn, elle venait matique des langues pwaamei et pwâpwâ, deux lan- gues du nord de la Grande Terre, qu' elle n’a pas eu le temps de finaliser pour publication avant son décès.

Pour conclure...

Connue pour sa passion des langues kanak et sa joie de vivre à la tribu, Françoise a toujours eu une conscience aiguë de la nécessité de transmettre et de sauvegarder ces langues souvent menacées. C'est ainsi qu'elle a associé ses colla quion des dicionn En En bonne disciple d'Haudricourt, et n'oubliant pas ses premières classes en ethnologie, elle s'intéressait aussi à la botanique, à la faune et à la flore et tous se dictionnaires, comme ceux de Jean-Claude d'ailleurs, comportent des nomenclatures de la faune et de la fore calédoniennes, utiles à la fois pour la compréhension de l'environnement et de la culture kanak, mais aussi pour les reconstructions des termes ancien en linguistique historique. Elle avait de grands talents de dessinatrice et n'hésitait pas à croquer, sur ses carnets, plantes ou oiseaux de Nouvelle-Calédonie. Jean-Claude Rivierre, de l'inventaire des langues
mélanésiennes à l'étude des tons des langues kanak

Jean-Claude Rivierre (1938-2018), quant à lui, travaillé sur les langues kanak bwatoo, cèmuhî, drubea, kwênyii, numèè, paicî, pije et xârâcùù. Il partageait beaucoup des centres d'intérêts de Françoise cités ci-dessus.

Jean-Claude Rivierre fut recruté au CNRS en qualité de stagiaire de recherche à partir du 01/11/1964 pour un an, sous la direction d'André-Georges Haudricourt. Mais, suite à diverses péripéties liées au service militaire ${ }^{40}$, il ne débuta son activité au CNRS qu’en janvier 1965 par une mission de deux ans et demi au cours de laquelle il rassembla des documents sur une douzaine de dialectes aux Nouvelles-Hébrides ${ }^{41}$ et sur les cinq langues à tons kanak en Nouvelle-Calédonie (cf. rapport d'activité de JCR de 1971)

Son cursus universitaire fut à peu près le mêm que celui de Françoise (et c'est, rappelons-le, duran leurs études qu'ils se sont rencontrés à Caen) : un Licence de lettres de la faculté des Lettres et sciences humaines de l'Université de Paris en octobre 1962 avec quatre certificats d'études supérieures : Sociologie générale, obtenu le 20 octobre 1961 ; Psychologie sociale, obtenu le 27 juin 1962 - Francoise l'a fait la même année - (avec une épreuve spécise la spéciale de Langue vivante ${ }^{42}$; Ethnologie, obtenu le 19 octobre 1962; Economie politique et sociale, obtenu le 28 octobre 1960

Les motivations de sa candidature, telles qu'il les a décrites, sont :

40. Pour résumer, il est rattrapé par les obligations du service militaire et, en dépit des démarches menées, il ne pourra pas être

ont été communiquées à des étudints ou professeurs étrangers (Nouvelle-Zélande Hawa'i). (Rappel dans soldes mais mes notes 42. Dans ses dossiers de candidature, il note : " anglais lu et parlé. 


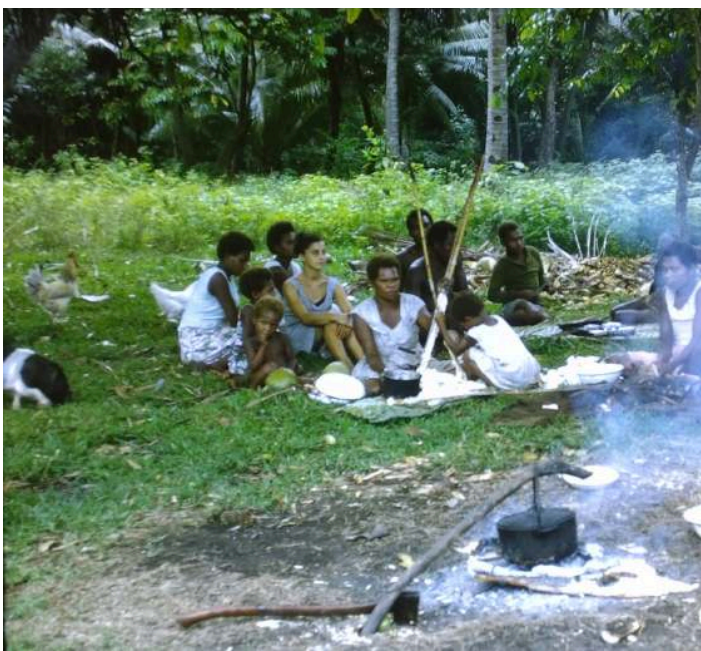

Рното 20. - Françoise sur le terrain au Vanuatu (1966) White Sand Betel (cliché CFr)

"dans le cadre de ma première année de doctorat de troisième cycle, étude phonologique en cours du kapone, dialecte parlé au sud de la Nouvelle-Calédonie par environ mille personnes. Ce dialecte connait trois ton mélodiques, quatre séries de consonnes et treize timbres vocaliques. Les séries consonantiques sont : sourde, semnasale, nasale et spirante sonore. Pour les points d'articulation : labial, labiovélaire, dental, affriqué chuintant prépalatal, affriqué chuintant cacuminal, vélaire, labiovélaire et unulaire. Tous les mots se terminent par une voyelle. Le système vocalique à linitial est un systeme réduit par rapport au systeme de la finale. Le système vocalique nasal est un systememe réduit par rapport au systeme oral. Toutes ces didature de JCR pour l'année 1963-1964, 15 mai 1963)

Tel était donc son programme de recherche au début de sa carrière dans le cadre de sa thèse. Pour cette candidature, son programme est aussi de " mener des Hébrides", ce Hébrides ", ce qui concorde avec la demande de Jea dré Martinet ${ }^{43}$ et d'André-Georges Haudricourt.

dré Martinet ${ }^{43}$ et d'André-Georges Haudricourt.
Le premier rapport de son directeur de recherche, Le premier rapport de son directeur de
A.-G. Haudricourt, précise début 1965 :

« M. J.-C. Rivierre est [un] chercheur à l'oreille particulièrement douée pour l'étude des langues à tons, il vient d'arriver en Nouvelle-Calédonie pour relever le système tonal des langues mélanésiennes, domaine absolument neuf dont los linguistes attendent beaucoup. Il doit aussi participer à RCP (Recherche coopérative sur protramme) de M. Gui en (Recherche coopérative sur programme) de M. Guiat e suis son travail depuis deux ans (il a pu traviller un per.

pendant son service militaire) et je pense qu'il devrait être promu au grade d'attaché de recherches. "

Lavis de son parrain André Martinet, en date du $1^{\text {er }}$ mars 1965, précise que :

"Monsieur Rivierre, qui est en mission en NouvelleCalédonie et aux Nouvelles-Hébrides, a commencél 'étude du cèmuhí, langue de Nouvelle-Calédonie et de la communauté qui la parle. Il procède en outre à lelaboration destiné aux enquêtes en Mélanésie. " [Voir Thomas et al., 1971 ; Rivierre, 1971]

En début d'année 1966, Haudricourt, son directeur au CNRS, note que :

«M. Rivierre a fait de l'excellent travail depuis son arrivée en Océanie. En Nouvelle-Calédonie, il a approfond textes, en complétant le vocabulaire (herbier envoyé textes, en completant le vocabulaire (herbier envoye en langue : à trois registres, type décrit pour la première fois langue : à trois registres, type décrit pour la première fois en Océanie. Aux Nouvelles-Hebrides
la RCP de M. Guiart, il a commencé l'exploration linguistique des îles centrales de l'archipel : Tongoa, Tongariki, Vate, Tanna ; dans cette dernière île au sud de l'archipel il a pu dresser une carte des langues et dialectes. Rapport très favorable. "

En février 1967, le rapport de direction de recherche signé par Haudricourt mentionne :

"M. Rivierre a brillamment réalisé son programme de recherches en Nouvelle-Calédonie : il a déterminé la tonologie de trois langues mélanésiennes, son enquête intensive et du maximum de vocabulaire de ces langues, menacées dans les jeunes générations, sera essentielle pour l'étude des problèmes posés par l'apparentement généalogique de ces langues à tons avec les langues sans tons qui les envionnent, et les problèmes de mutation consonantique que M. Rivierre a d'ailleurs résolu[s] pour la langue de l'extrême sud. J'ai pu, au cours de ma mission en juin-août 1966, apprécier lexcellence de ses méthodes sur le terrain. 'est d ailleurs ma présence qui l'a empêché de retourner aux Nouvelles-Hebrides pour le programme d inventaire linguistique, mission quil fera cette année avant son retour. Rapport très favorable, promotion si possible. "

D’août 1967 à février 1968, dans le cadre de sa thèse de $3^{e}$ cycle ayant pour sujet les dialectes du sud de la Nouvelle-Calédonie, Jean-Claude procède à la mise en fiche du dialecte de l'île des Pins, le nââ kwênyi (2 000 fiches/termes) et à l'étude de quelques textes enregistrés avec mise au propre avec notation tonale

" Mais le système tonal de cette langue ne peut être élucidé sans une connaissance approfondie de la tonologie

43. André Martinet (1908-1999) est connu comme le père de l'analyse fonctionnaliste en linguistique française. De 1938 à 1946 il a enseigné comme directeur d'études à l'EPHE, puis à l'Université de Columbia où il fut nommé directeur du département de linguistique (1947-1955). De retour à Paris en 1955, il revint à l'EpHE, fut titulaire de la chaire de linguistique générale à la Sorbonne,
puis à l'Université Paris $5-$ René Descartes. Président de la Société européenne de linguistique de 1966 à 1999 , il fonda la Société de puis à 'Université Paris 5-René Descartes. President
linguistique fonctionnelle et la revue La Linguistique.

44. On peut se demander ce qu'est devenu cet herbier et la part de For dans sa constitution, elle qui était passionnée de botanique, 45. Joite ligne d AGH (voir photos 18-19 sur les sorties botaniques). Je n'en ai mahlesuresement trouvé aucune trace.

Nouvelles-Hébrides mais mes notes ont été communiquées à des étudiants ou professeurs étrangers (Nouvelle-Zélande, Hawa' i) (rapport de 1971 de JCR). Voir aussi Rivierre (1968, 1969a-b). des dialectes du Sud. Nous avons donc repris la question nous établissons la phonologie complète, en procédant notamment à la mise en fiche et à l'étude tonale des textes
recueillis. » (Extrait du rapport d'activité de 1967)

Dans son rapport d'activité de 1967, il énonce que

«Le dialecte de l'île des Pins est très proche du dialecte parlé à l'extrême sud de la Nouvelle-Calédonie (GoroTouaourou) ; à l'inverse du dialecte d'Unya-Paita (dont le consonantisme seul est différent) il en differe par le vocalisme et le système tonal [...]."

Le rapport d'Haudricourt en tant que directeu de recherche pour l'examen en commission en ma 1968 précise que :

" M. Rivierre, depuis la fin de sa mission d'enquête sur le terrain, soccupe de transcrire et d etudier les textes quil une thèse avec M. Martinet. La qualité des matéria une thèse avec M. Martinet. La qualité des matériaux
recueillis et l'originalité de ces dialectes au système tona recueillis et loriginalité de ces dialectes au systeme ton à deux registres
très favorable. "

Le rapport de son parrain Martinet complète le précédent :

«M. Rivierre a su compléter, seul, une formation linavaient un peu écourtée. Il est aujourd'hui un très bon descripteur dont le travail et les initiatives sont parfaitement satisfaisantes. On peut attendre de lui des présentations scientifiques très sûres des langues du sud de la Nouvelle-Calédonie. On recommande le renouvellement et la promotion éventuelle pour ce chercheur.

Son rapporteur, M. G. Manessy, émet donc un avis très favorable au renouvellement ; le contrat de JeanClaude n'est prorogé qu à la session d'octobre, avec renvoi à lavis émis par le rapporteur en mai, retard sans doute dû aux événements de mai 68 . tion comme chargé de recherche :

"M. Rivierre continue à élaborer les matériaux quil a mine la rédaction d'une thèse de $3^{e}$ cycle sur les dialectes travail est la première description détaillée d'une langue mélanésienne tonale dans toute la littérature linguistique, il fera date dans la linguistique océanienne. Les textes de litterature orale et les dictionnaires des trois dialectes sont en voie de laboration pour publication. Lensemble du travail fait, tant par sa qualité que par son ampleur, justi-
fierait une promotion à chargé de recherche. M. Rivierre en partant du dialecte le plus simple : celui d'Unya, dont Calédonie. Il doit en tirer une étude phonologique pour guistique que les besoins de la recherche sur le terrain

En 1969, son directeur AGH demande une promorecueillis dans sa fructueuse mission de 1965-67. Il tede l'extrême sud néo-calédonien [Rivierre, 1970]. Ce

aura ensuite à s'occuper des langues à tons de la partie centrale de la Nouvelle-Calédonie, dont il a recueilli ou vérifié un corpus important de textes et de dictionnaires, commande donc vivement renouvellement et promotion de M. Rivierre. "

L'avis de son parrain, André Martinet, est tout à fait concordant :

« [...] Les résultats sont excellents. On peut attendre de cet excellent chercheur qu'il nous donne enfin un table scientifique complet de la situation linguistique très complexe de cette île [Nouvelle-Calédonie]. Il est indispensable que M. Rivierre soit assuré du renouvellement $d e$ son contrat jusquà l'aboutissement de ses travaux.

René Gsell146, célèbre phonéticien et rapporteu pour la session de mai 1969, conclut donc par un avis très favorable pour le renouvellement ${ }^{47}$ et la promotion :

« Monsieur J.-C. Rivierre a poursuivi cette année ses traval té étudiés du point de vue paradigmatique et syntagma tique avec examen du mécanisme des assimilations et des dissimilations tonales ainsi que celui des neutrasistions. De plus, Monsieur Rivierre a [terminé] la rédaction de sa thèse de troisième cycle. "Dirlectes de l'extrême sud calédonien ; phonologie descriptive et comparative" Excellents rapports de Monsieur Haudricourt et de M. Martinet. Lensemble du travail fait, tant par sa qualité que tinet. Lensemble du travail fait, tant par sa qualite que par son amp
recherches. .

En 1970, André Martinet, parrain et directeur de thèse de Jean-Claude, mentionne dans son rapport qu il vient de lui présenter pour soutenance :

" une excellente étude de la phonologie des langues de extrême sud-est de la Nouvelle-Calédonie. Il y fait preuve matière d'ethnologie du Pacifique Sud-Ouest. Il est triès important pour la recherche linguistique et anthropoloqique que Monsieur Rivierre poursuive ses travaux et l'o recommande pour lui une promotion si son ancienneté son classement le permettent. "

Ce rapport concorde totalement avec celui de son cirecteur de recherche qu est Andre-Gorges Haudricourt qui, a propos de son manuscrit de these deposé, signale que :

"Ce travail de pionnier sur les premières langues à tons décrites de Mélanésie fera date dans l'histoire de la linguistique océanienne. Je rappelle, qu'outre ce travail, J.

46. René Gsell (1921-2000) était un linguiste et phonéticien français. Assistant de phonétique en 1955 à la faculté des lettres de rale appliquée (ILPGA) dont il a largement contribué au développement et qui est aujourd'hui rattaché à l'Université Paris 3 Sorbonn nouvelle.

47. Cette session de mai 1969 a une importance particulière. Jean-Claude est au terme de quatre ans d'ancienneté dans le grade d'attaché de recherche et une note précise que le renouvellement pour une nouvelle période de deux ans doit être accordé ou refusé et que : " mememe parmi les attachés à quil la section souhaiterait qu un $3^{*}$ contrat de deux ans soit maintenant accorde, il peut sen trouver un certain nombre dont la stabilisation a terme dans les cadres du CNRS n apparaisse pas souhaitable avec évidence. Ces derniers, en vertu de la note de

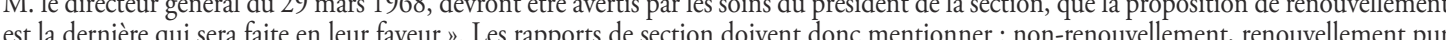


a élucidé le problème, plus simple, des autres langues à tons de lîle, et recueilli un important matériel de littérature orale. J'ai montré dans un article (BSL 63, 1, 218$\left.235^{48}\right)$, à propos d'une de ces langues, la langue de Touho, l'intérêt que présentaient ses observations pour expliquer
la naissance de langues à tons ponctuels. En résumé, les travaux publiés et inédits de ce chercheur me permettent de recommander vivement sa candidature au grade $\mathrm{d}$ chargé de recherches. Je recommande également la pris en considération de sa demande de mission pour retouner quelques mois en Nouvelle-Calédonie,

C'est à nouveau René Gsell qui rapporte pour session de mai 1970 sur le dossier de Jean-Claude. Il mentionne que sa thèse, dont JCR a fait une presentation de 18 pages comme rapport d'activité de l'année 1969, fera date dans l'histoire des recherches de tonologie :

« Monsieur Rivierre y décrit entre autres le mécanisme de a naissance des langues à tons ponctuels. [...] En raiso de l'intérêt exceptionnel de ses travaux, je recommand très vivement non seulement le renouvellement de Monsieur Rivierre mais sa promotion au grade de chargé de recherches. Je donne également un avis extrêmement favorable à sa demande de mission en Nouvelle-Calédonie. "

Dans son rapport d'activité pour l'année 1970 Jean-Claude présente l'avancée de ses travaux suite à sa thèse sur les langues de l'extrême sud, avec la mise au point des textes recueillis à Goro et Touaourou avec vérifications des transcriptions faites sur le terrain, notation des tons, traduction et étude de la toponymie (soit 256 pages manuscrites). De même, il commence à remettre au propre et à refondre fichier établi sur le terrain. Puis, il prépare sa missio de terrain prévue pour juin 1971 pour réliser une

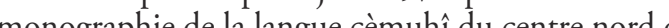
monographie de la langue cemuhî du centre nord de la Nouvelle-Caledonie. Il etablit notamment un tichier des toponymes cités dans les textes et les reporte
sur une carte au $1 / 50000^{\circ}$. On ne peut que regretter sur une carte au $1 / 50000^{\mathrm{e}}$. On ne peut que regretter
que cette carte ne figure pas dans ledit rapport.

C'est à nouveau Alexis Rygaloff qui rapporte sur son dossier en 1972 ; il émet à nouveau un avis très favorable :

«M. Rivierrre a eu une année particulièrement bien remplie. Sur le terrain où il a passé 6 mois (mai à octobre pl... Un questionnaire de 1500 phrases a été rempli [...], plusieurs heures de discours cérémoniels inédits enregis-
trées sur bandes magnétiques. Plusieurs articles en cours trées sur bandes magnétiques. Plusieurs articles en cours
de rédaction, et un article achevé en collaboration avec de rédaction, et un article achevé en collaboration avec
M. Salva [sic] du Muséum, à paraitre prochainement ${ }^{49}$. M. Salva [sic] du Muser
(session de mai 1972)

À propos de la langue de Touho, Jean-Claude précise, dans son rapport de 1971 à propos de la littérature orale, que :

«Dans le domaine des contes, mythes, etc., j’ai constaté que javais fait le plein ou presque lors de mon premie passage - avec raison et in extremis puisque tous les vieux conteurs sauf un sont morts depuis. Mais dès mon arrivée, j'ai été invité à suivre de bout en bout un mariage puis, peu après, un enterrement. J'ai pu ainsi enregistre trois ou quatre heures de discours cérémoniels dont je ne soupçonnais même pas l'existence et, ce qui n'était pas prévu à mon programme, j’ai pu passer un mois et dem à les transcrire. Ces textes faisant de très nombreuses références à la sociologie locale, j’ai dû amorcer une enquête sur les clans de la région pour pouvoir les comprendre. Il ma paru interessant de faire ce travail car il s'agit de discours traditionnels, formalises, riches en images, mots et tournures que je navais jamais entendus. En outre, ces discours sont importants pour la comprehension de lethnologie calédonienne et pour lethnologie politique

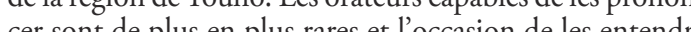
nest paś n'est pas fréquemment offerte. Or aucun texte de ce genre

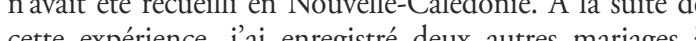
cette expérience, $j$ ai enregistré deux autres mariages traditionne en langue paî. Au cours de cette nission, j’ai enregistré et transcrit une vingtaine de poésies, contes et histoires de clan. Au total je me suis retrouvé en contes mission avec davantage de textes et donc de problèmes qu'au départ alors que je comptais clore la révision de mes corpus et faire l'indispensable toponymie. C'est avec cette ferme intention que je repartirai là-bas pour dest avec cette ferme intention que je repartirail la-bas pour deux ou trois

C'est là le propre de la recherche que de devoir se remettre en cause en permanence, au gre des terrain et de là où ils nous mènent sans que nous l'ayon réellement prévu. Cet extrait nous donne toute l'ampleur du travail de Jean-Claude qui va bien au-delà de la seule linguistique, avec des documents proprement anthropologiques. Il poursuit

« Reste le problème du paicî, langue à tons étudiée lors de ma première mission. Cette ethnie est de loin plus dynamique, la plus créatrice et en même temps mieux conservee de Nouvelle-Caledonie. J ai eu la surprise d obtenir cet été, sans recherche particulière, un enregis trement dune poésie de 1500 vers. Il est nécessaire d'entreprendre prochainement le ratissage de cette région en matière de litterature orale; une telle entreprise ma pris beaucoup de temps dans une région beaucoup plus délabrée comme celle de Touho. Je compte lentreprendre ave dans le courant 73 . En attendant, je lui apprends ce que je connais de cette langue tous les jeudis. » (Extrait du rapport 1971 de JCR)

Dans son rapport d'activité de l'année 1976, note :

"Le problème des tons étant ma véritable spécialité linguistique [voir Rivierre, 1972a-c, 1974a], mon directeA. Haudricourt ma demandé de passer par le Mexique, sur la tonologie des langues pame (famille otomangue). Dans Mac Donald sures documents qu'lle a enich l'an dernier chez les Mixtèques (de cette même famille

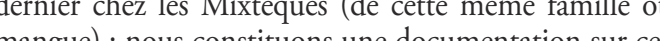
famille ; nous concus

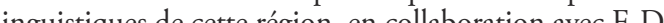
linguistiques de cette région, en collabors
qui s'intéresse aussi à ces systèmes tonals.

Jean-Claude est désormais membre du tout nouveau LACITO fondé par Jacqueline Thomas (LP3121 localisé à Ivry) au sein duquel il est responsable de la section Océanie. En fin de rapport, avant quatre pages sur les paradigmes directionnels en Mélanésie (à partir $\mathrm{du}$ cèmuhî), il fait une remarque que d'aucuns jugeront toujours d'actualité :

"J'ai tout lieu de me féliciter de cette création, eu égard aux nouvelles conditions de travail qu'elle nous procure. Cette année n'en a pas moins été marquée comme la précédente par un renforcement de la tutelle administrative qui pèse sur la recherche, corrélatif d'une moindre efficacité de cette administration et d'une baisse des crédits globaux. Cet état de fait amène une perte de temps et d'éne gie de plus en plus importante en démarches, réunions, actions, réclamations ${ }^{1}$, etc.

Suite à une rencontre imprévue à Paris ${ }^{52}$, il changea son programme de recherche pour la mission q devait avoir lieu du 31 août au 11 novembre 197 et porter sur l'étude de l'expression linguistique de l'espace sur les langues de la côte est et de la côte ouest afin d'aller à Thio et Canala. À la place, il réalisa, durant le mois d'octobre, une esquisse grammaticale à partir du questionnaire verbal de l'ouvrage collectif de l'ER74 Enquêtes et description des langues à tradition orale, ce qui lui permit d'appréhender le système prosodique, de souligner l'absence de ton mais la présence de proclise et d'enclise entraînan un conditionnement morphologique / grammatica dans cette langue.

50. Il s'agit d'Alban Bensa (1948-) qui fut introduit par Jean-Claude sur le terrain calédonien, comme il en sera fait mention dan les rapports suivants. À l'époque, il était assistant à l'Université Paris 5-René Descartes. Il devint maître de conférences, toujours

51. On peut supposer que ce mouvement d'humeur est dû au fait, comme le note Alexis Rygaloff dans son rapport de mai 1977 quasi suppression de la subvention accordé jusq'à pésent par le cNRs w. Ladite subvention fut rétblie, commele note Jean-Chude dans son rapport d'activité de 1977, après la session de mai, « suite à l'intervention de M. Rygaloff et à celle de M. le directeur scienifique ". On peut remarquer quil mentionne qu'« en raison de la pénurie de crédits, je participe moi-même à la frappe des textes sur machine Івм, en vue de la publication en offset ». Tout un monde que les chercheurs d' aujourd hui n'envisagent même pas. . 52. Mission en Nouvelle-Caledonie. " [...] ce programme a eté remis en question en juin [1977], tout à fait par hasard, suite à ma rencontre avec quelques Néo-Calédoniens de passage à Paris. Lors de cette rencontre, j'a pu punstater avec stupéfaction, en les écou-

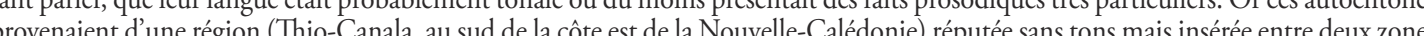
provenaient d'une région (Thio-Canala, au sud de la côte est de la Nouvelle-Caledonie) réputée sans tons mais inserée entre deux zones 1977). L'un d'entre eux était le grand chef de Thio, Damas Toura qui faisait partie de cette délégation sLv (Société Le Nickel). 
les matériaux lexicographiques et sur les traditions orales, travail qu'il avait entamé dès 1985 :

«Le projet de publication de ces matériaux recueillis il y a plus de vingt ans répond au souhait des locuteurs, plus particulièrement à l'un de leurs représentants, Clément Vendégou, maire de Yaté, avec qui une partie de ces matériaux a été révisée récemment. À l'occasion de ses différents déplacements entre le territoire et la métropole, j’ai pu terminer avec lui la révision du lexique en langue de Goro, refaire et mettre au point le fichier avec les précisions de traductions, les corrections phonétiques, les exemples, etc. en vue d'en reprendre la publication Ont été revues et classées par familles les nomenclatures (poissons, coquillages, oiseaux, etc.) pour les faire figurer en annexe du dictionnaire, selon le mode de présentation déjà adopté pour les autres dictionnaires $5^{53}$. A plus long terme, le but envisagé serait la publication de toute cette do texte, en faisant figurer à côté de chaque entrée en de texte, en faisant figurer à coté de chaque entrée
langue de Goro, les équivalents dans les différents dialectes de l'extrême sud (Unya, Paita, Wêê, île des Pins). Grâce aux opérations de tri réalisées par ordinateur, il devrai etre possible de produire des dictionnaires de ces differents dialectes, avec à chaque fois, les équivalents dans les de l'extrême sud. " (Extrait du rapport 1987-1988 de JCR, repris dans celui de 1987-1991)

Le décès de Clément Vendégou dans un acciden de voiture sur la route de Nouméa à Yaté (le 18 juin 1999) est venu contrecarrer ce projet qui ne fut donc jamais publié. Nous l'avons retrouvé en préparant ce volume et nous l'avons déposé sur la plateform volume et nous lavons déposé sur la plateforme HAL archives ouvertes du CNRS en aout 2020 (ht

halshs.archives-ouvertes.fr/halshs-02916886).
Une autre partie de son programme de travail pour cette année consista en l'avancement de recherch comparatives sur les langues de l'archipel néocalédonien grâce aux travaux menés à l'époque par Françoise Ozanne-Rivierre sur les langues du nord de la Grande Terre et celles des Loyautés.

«Une première approche du problème, réalisée par G.W. Grace sur deux langues (Canala, Tîrî̀) a mis e evidence un très grand nombre de correspondances irregulières et fort peu de cognates rapprochables de faço sưre de POC (proto-océanien). La seule façon de dépasse sembler le plus de documentation possible, notamment enté le plus de docunntation possible, notamment en préparant soigneusement une enquête sur cinq langues Nonver du rapport 1987-1988 de JCR)

Depuis la fin des années 1980, Jean-Claude s'intéressait plus particulièrement à la phonologie compa-

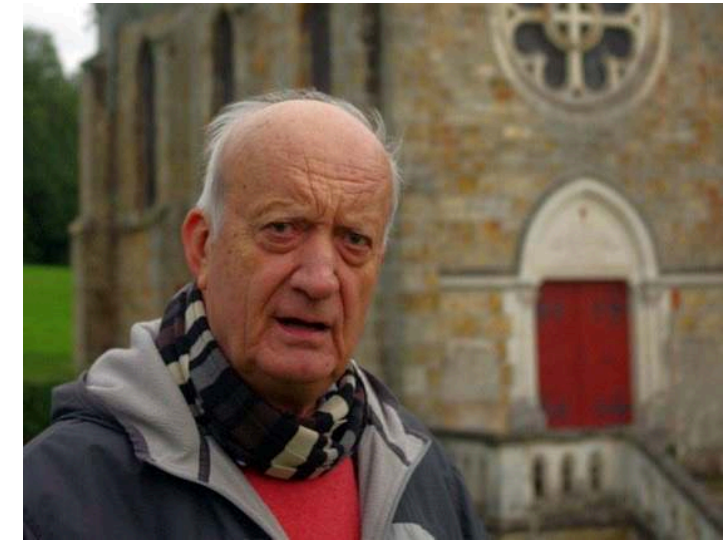

Рното 21. - Jean-Claude Rivierre en 2013 devant l'oratoire. construit à la fin du XIX siècle, de Port-Royal des Champ commune de Magny-les-Hameaux (cliché François)

rative (langues néo-calédoniennes et océaniennes) et à la phonologie diachronique (Nouvelle-Calédonie), sans oublier la tonogénèse, «sa spécialité » si je puis dire.

"Vers 1987, un infléchissement de ses recherches en faveur de la diachronie et du comparatisme dont la multiplicité des langues locales suggère l'étude [...] [d'un] domaine [dans lequel], au cours de ces quatre dernières années, il a apporté les contributions les plus remarquées. [... On retiendra peut-être tout particulièrement ce que J.-C. Rivierre a pu révéler des modalités d'apparition des voyelles nasales et des répercussions induites sur le consonantisme. » (Extrait du rapport de Blanche-Noëlle Grunig pour la période 1988-1991)

Au début de son rapport, B.-N. Grunig note qu'il semble que Jean-Claude Rivierre n'ait que très rarement fait acte de candidature [à DR1] alors qu'il est «depuis seize ans (1976) maître de recherches (équivalant DR2) ».

L'année 1996 marque la fin du département Océahie - langues et cultures du Pacifique et Jean-Claude est désormis rattaché a l'équipe stypologie et chanement linguistique» (voir Leblic et Masquelier, 2020 ; Souag, 2020).

Dans son rapport d'activité à 2 ans de juin 1997 Jean-Claude précise qu'il a terminé la saisie du dictionnaire de la langue de Goro-Touaourou sur shoe$b x^{54}$ et que l'ensemble existe donc sous la form d'un dictionnaire numèe-français accompagné d'u index inversé et des différentes nomenclatures $d$ monde naturel ${ }^{55}$. Il reste juste à y entrer les notation tonales, les morphèmes grammaticaux et les équivalents pour chaque entrée dans les langues et dialectes

53. Une version précédente de ces nomenclatures en ma possession a été mise en ligne sur HAL archives ouvertes du CNRS (https:// halshs.archives-ouvertes.fr/halshs-03091799) en tant que document de travail, comme pour le dictionnaire déjà mis en ligne. 54. Sur le site du LLACAN, on peut lire : « Logiciel de gestion de bases de données textuelles édité par la sIL (http://www.sil.org). I permet la saisie, l'affichage, la recherche, le tri, le filtrage, limpression... de fiches constituées de textes structurés en champs. Plusieurs bases peuvent être ouvertes en même temps et des liens peuvent s'établir entre elles. Il est particulièrement adapté à la gestion de fiches même fiche (sous-entrées, différents sens, plusieurs phrases illustratives avec traduction...). , (http://llacan.cnrs.fr/fichiers/monels Shoebox/ShoeboxInit.htm)

55. Je n’ai malheureusement trouvé aucune trace de ce fichier shoe-box dans les archives de Jean-Claude.

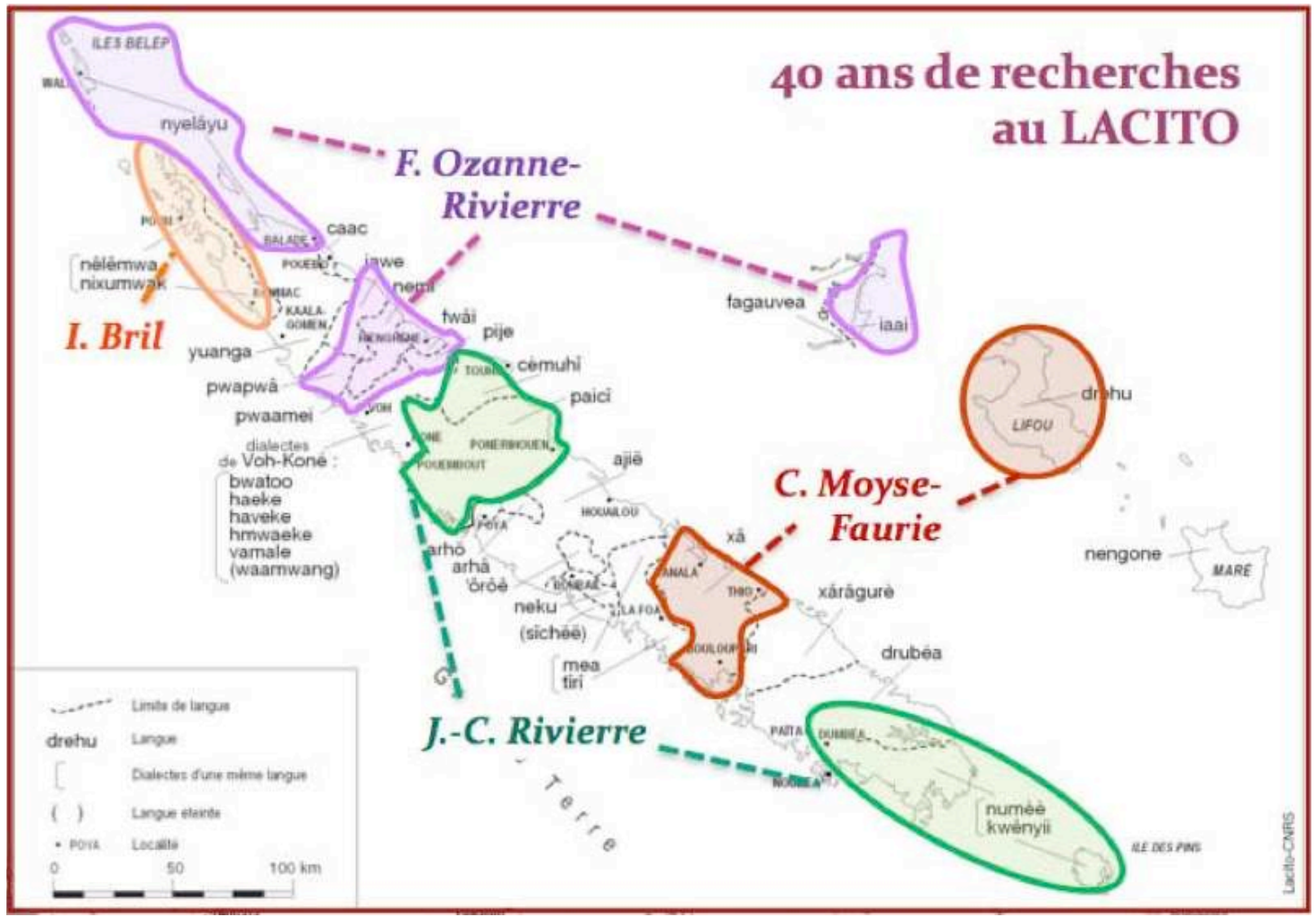

CARTE 1. - Carte linguistique des langues kanak précisant les recherches de Françoise Ozanne-Rivierre et de Jean-Claude Rivierre, ainsi que celles de Claire Moyse-Faurie et Isabelle Bril, les collègues qui sont venues à leur suite travailler au LACITO sur les langues de Nouvelle-Calédonie (@) CNRS-LACITO)

de l'extrême sud, à savoir, 1. Goro, Ouen ; 2. île des Pins ; 3. Unya, Paita. Outre ce dictionnaire, côté lexicologie, il a participé au projet MARIAMA, logiciel pour élaboration de dictionnaires, recherches thématiques, comparaison de langues, etc. Le logiciel a été appliqué pour les nomenclatures botaniques. Autre thème pour ces deux années, la linguistique historique, pour laquelle Jean-Claude a repris l'étude des consonnes labiovélaires néo-calédoniennes suite aux travaux pionniers d'Haudricourt. Puis, il a travaillé sur l'évolution de la structure syllabique des langues néo-calédoniennes avec F. Ozanne-Rivierre. Le dernier grand thème consiste dans la poursuite de l'ethnolinguistique, de l'étude des traditions orales et de l'histoire locale, travail qu'il menait depuis de nombreuses années avec Alban Bensa, mais qui n'avait pas encore abouti.

En 1998, Jean-Claude participe à deux actions de valorisation: il devient membre du comité de réflexion du programme européen d'action contre l'illettrisme dans l'entreprise et consultant de la Délégation générale de la langue française (DGLF), en vue de la signature de la charte européenne sur les langues régionales, et du ministère des DОМ-TOM et élabore des documents sur les langues de NouvelleCalédonie (voir JCR, 2003)

En 2001-2002, la dernière fiche documentaire qu'il remplit avant son départ en retraite, le 19 juin 2003, nous apprend qu'il est toujours DR2 et que les points forts de ses activités sont la lexicologie, la tonologie a littérature orale et la morphosyntaxe.

Le 21 décembre 2007, il est nommé chevalier de l'Ordre des arts et des lettres par Christine Albanel, ministre de la Culture et de la Communication.

Principales contributions théoriques de Jean-Claude

Tout au long de sa carrière, en matière de recherche inguistique, Jean-Claude a poursuivi en parallèle l'étude de plusieurs thématiques :

- la tonogénèse, qui est sans conteste sa spécialité avec l'élucidation pionnière de la tonologie des langues kanak que sont le cèmuhî - à trois registres, une première en Océanie -, le paicî et les langues du Sud (JCR, 1972a-b-c, 1973a, 1974a, 1975, 1978 c. 1979a-b, 1980, 1983, 1993, 1994a, 2001b)

- l'importance de la tradition orale dans l'étude des sociétés kanak qui l'a poussé à collaborer avec un 1971, 1996 ; JCR et a 1980, 2005, 2011 ; B JCr JCR, 1976, 1982, 1983, 1984, 1988a-b, 1990, 1995 Capo et JCR, 2012)

- la phonologie comparative et la phonologie historique, domaine dans lequel FOR et lui-même on grandement collaboré (JCR, 1968, 1969b, 1970 1991a, 1994b, 1995, 1997, 2000a, 2011; FOR et JCR, 2004a-b-c) ;

- la politique linguistique et l'enseignement des langues kanak (Haudricourt et al., 1979; JCR, c. 1981, ethnologue, Alban Bensa, qu'il a initié au paici (JCR, 
1981, 1984, 1985, 1991b, 2001a, 2003 ; FOR et al., 1988 ; JCR et al., 2001-2002; Moyse et JCR, 1991, 1992 ; Moyse et al., 2012).

- les questionnaires d'enquêtes linguistiques, les dictionnaires de langues kanak et l'ethnoscience JCR, 1971, 1983, 1994a, c. 1994 ; JCR et Vendégou 1997 ; FOR et JCR, 2004b ; JCR et al., 2006 ; Thoma et al., 1971; Jacobson et al., 2001)

À cela, il faut aussi ajouter les recherches faites avec Françoise et déjà mentionnées ci-dessus, notamment sur la reconstruction du proto-calédonien. Et, comme déjà mentionné, ses recherches fondamentales, comme celles de Françoise, ont été menées de front avec les recherches appliquées afin d'aider l'enseignement des langues kanak.

\section{Et pour conclure}

Jean-Claude n'a cessé de remettre sur le métier des chantiers restés inachevés au début de sa carrière, comme les dictionnaires sur les langues du Sud, travaux qui sont revenus régulièrement au fil des années. Malheureusement, le dictionnaire de numèè, sur lequel il a retravaillé à de nombreuses reprises, les dernières fois avec Clément Vendégou - qui tenait à sa réalisation pour permettre aux jeunes génération de continuer à avoir accès à la langue des anciens est resté au stade de manuscrit suite au décès prématuré de Clément. Durant mon tertain à Goro 1985-1986, cest chez Clém nue j'habi à C' 1985-1986, c'est chez Clem ment que jhabitais. C'est sans doute à cette occasion quils en sont venus mettre leurs forces en commun, car j avais apporté une version dactylographiee du premier dictionnaire numèè. Or, Jean-Claude avait travaillé notamment avec Isaac Vendégou à Touaourou, un des "papas" grand-père d'Alphonsine, épouse de Clément.

Il m’est arrivé, durant mon terrain à Goro début 1986, de circuler avec lui auprès de plusieurs de ses anciens interlocuteurs et j'ai pu apprécier commen il avait marqué la mémoire de tous les gens avec qui il avait travaillé J'ai aussi vu nombre de Kanec étuliants en Fance et des gén diants en France et des gênéntions venir rencontr. au LACHO pour récupérer les enregistrements faits auprès de leurs a vienx " souvent decéd depuis lors. A l'époque, les choses n'etaient pas numérisées ni disponibles en ligne comme elles le son maintenant. Et, inlassablement, il refaisait des copies à qui le lui demandait..

Ainsi, de génération en génération, les relations se tissent et se reproduisent de façon souvent inattendue. C'est suite à ma rencontre avec Clément Vendégou à Paris en 1984, après avoir travaillé pour mon premier terrain dans le Sud sur les clans pêcheurs kanak quill 'incita à venir chez lui à mon retour sur le terrain. Mes deux premiers terrains furent donc coment Mes deux premiers terrains fur rent done communs houen, de langue paicî, autre terrain de prédilection 56. Certains thèmes sont restés sans propositions, comme par exemple la syntaxe comparative, la typologie, la tonogénèse..., les
Pour donner toute leur place aux recherches des Rivierre, outre la présentation de leurs parcours que nous venons de voir et la reconstitution de leur bibliographie faite ci-dessous, une recherche leur bibliographie faite ci-dessous, une recherche de ficelle kanak est d'abord présentée et commentée par moi-même, puis par Agnès Henri et Henri Vandendriessche.

\section{Les jeux de ficelle en Nouvelle-Calédonie}

Ce dossier commence par deux articles liés à un corpus de jeux de ficelle collecté en Nouvelle-Calédonie par Françoise Ozanne-Rivierre dans les années 1965-1967 et retrouvé dans les archives de JeanClaude Rivierre après son décès en janvier 2018. C corpus de plus de cinquante jeux de ficelle recueillis dans l'aire paicî-cèmuhî vient enrichir très utilement dans l'aire paicî-cèmuhî vient enrichir très utilement les rares publications existantes portant sur les jeux
de ficelle kanak. L'article d'Agnès Henri et d'Éric de ficelle kanak. Larticle d'Agnes Henri et d'Eric Vandendriessche propose tout d'abord une mise en contexte du travail de Françoise Ozanne-Rivierre, te qu'il nous est parvenu au travers de quelques carnets de notes sur les jeux de ficelle, accompagnés d'un texte dactylographié et manuscrit. Outre la présentation du corpus recueilli, l'étude de ces documents permet de préciser la méthodologie mise en ouvre par la chercheure. La seconde partie donne un aperçu des procédures impliquées dans ce corpus, en présentant étape par étape deux (séries de) jeux de ficelle, illustrées par des photographies, dont certaines réali-

illustrees par des photograph
sées par les époux Rivierre.

Pour ma part, j'ai choisi d'éditer ici, à titre po thume, les notes inédites de Françoise Ozanne-R vierre qui ont servi de base à l'article d'Agnès Hen et d'Éric Vandendriessche. Quelques éléments épa dans les divers documents constituant le fonds des travaux de Françoise Ozanne-Rivierre sur les jeux de ficelle ont été ajoutés à ce manuscrit par un appareil de notes explicatives conséquent que j'ai constitué. Pour ce faire j'ai consulté des Kanak de la région, dont Élie Poigoune, de la tribu de Poyes a région,

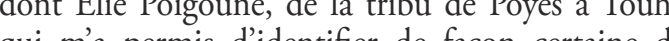
qui ma pentis didentifes de façon certaine des personnes presentes sur les photos. Il a aussi clarifié quelques mots à mots cèmuhî, qu il en soit ici à nouveau remercié. Ce travail de reconstitution mont que seule une connaissance précise du terrain et des langues permet de tirer le maximum des notes de Françoise Ozanne-Rivierre en rétablissant notamment de mauvaises attributions de langues ou des traductions imprécises.

Linguistique historique en Nouvelle-Calédonie, au Vanuatu et aux îles Bismarck

Toujours sur la Nouvelle-Calédonie, Isabelle Bril nous propose un texte qui a, entre autres, le mérite Rivierre, dans la droite ligne d'Haudricourt), archivage et retour aux in
littérature orale dans quinze langues de Nouvelle-Calédonie avec l'ADCK.

57. Autre écriture de la langue aneityum mentionnée plus haut (comm. pers. I. Bril) de s’appuyer sur les travaux antérieurs de Françoise Ozanne-Rivierre et de Jean-Claude Rivierre. Après un DEA sur la langue de Mono-Alu (Bougainville), sabelle Bril avait rencontré les Rivierre pour définir un terrain d'enquête linguistique sur une langue kanak de la Nouvelle-Calédonie dans le cadre d'une thèse co-financée par le LACITO et le programme de recherche ESK (Études des sociétés kanak) sous l'impulsion de Daniel de Coppet. La thèse d'I. Bril sur "La structure de l'énoncé en nêlêmwa" (extrême nord de la Nouvelle-Calédonie, soutenue en 1995) fut le début d'une longue collaboration avec les Rivierre au LACITO, avec une publication commune avec Francoise Rivierre sur les prédicats complexes en 2004. Dans la droite ligne des recherches faites par ses aînés, André-Georges Haudricourt Rivierre, qui ont montré que ces langues, longtemps considérées comme "aberrantes ", font partie intérante des langues océaniennes de la famille austronésienne, Isabelle Bril étudie ici de manière approfondie es processus évolutifs dans deux langues de l'extrême nord de la Nouvelle-Calédonie, le nêlêmwa-nixumwak et le zuanga-yuanga, langues sur lesquelles elle a conduit des enquêtes de terrain. Elle montre que leur structure syllabique est généralement mieux préservée, bien qu'une forte érosion syllabique soit aussi l'œuvre, plus particulièrement en zuanga de Gomen où toutes les consonnes finales ont chuté. I'inventaire des consonnes a égamén été réduit en compair comparadiverses a reconstrut de diverses neutralisations doppositions, en revanche, le zuanga de Gomen a conservé une opposition perdue illeurs entre les consonnes dentales et rétroflexes.

John Lynch, pour sa part, analyse aussi dans une perspective diachronique les accrétions pré-radicales en anejom ${ }^{57}$, une langue du Vanuatu. Cette langue océanienne, membre du sous-groupe sud-Vanuatu, fixé, par accrétion, une forme dérivée de l'article proto-océanien *na à la plupart des noms, et une voyelle à la plupart des verbes. Le reflet de *na est phonologiquement conditionné, selon que la première voyelle de la racine était *a ou une autre voyelle. Mais le constat ainsi formulé connait de nombreuse exceptions. John Lynch discute donc ici de ces cas, exceptionnels en apparence, et établit que la plupar d'entre eux peuvent être expliqués par une séquence de règles régulières. L'accrétion de *na à la racine $\mathrm{d}$ nom est attestée dans de nombreuses autres langues du Vanuatu, alors que l'accrétion verbale ne se produit que dans les langues du sous-groupe du Vanuatu méridional et ne semble pas dériver d'une forme reconstruite de POc.

Dans son article " On rank and leadership in proto oceanic society", Andrew Pawley étudie la diffusion initiale des langues océaniennes, au-delà de l'archipel des îles Bismarck et à travers le sud-ouest du Pacifique à l'époque Lapita, autour de 3000 avant notre 
ère. La question se pose de savoir quelles formes de pouvoir et d'organisation sociale ont sous-tendu l'expansion dorganisation sociale ont sous-tendu combiné à l'ethnophie comparaive peut ique comber nous Wairer. Cest aussi la question qu abordent Marie Walworth et Aymeric Hermann (voir ci-après). Larticle de Pawley presente un ensemble de données en faveur de l'hypothèse selon laquelle la société protoocéanienne était formée de groupes de descendance hiérarchisés, ayant à leur tête des chefs au pouvoir héréditaire appelés *ta(u)-lapat, et où le rang était basé sur la primogéniture.

Mary Walworth est la dernière à être venue au LACITO pour travailler avec Jean-Claude; pendan plusieurs mois, ils ont repris ses anciennes données et il était donc important pour Mary de participer a ce volume. Aymeric Hermann et elle proposiper ce volume. Aymeric Hernans et elle proposent un du Vanuatu central qui tente lui aussi de combiner plusieurs approches, linguistique, anthropologique plusieurs approches, linguistique, anthropologique
et archéologique, à partir de la méthode de trianet archéologique, à partir de la méthode de trian-
gulation de P. Kirch et R. Green. Comme le dit ici gulation de P. K
Andrew Pawley

"The archaeologists Kirch and Green (2001) draw on linguistic, archaeological and ethnographic evidence to reconstruct elements of 'ancestral Polynesian society'. " (ce vol., p. 246)

Cette méthode qui croise trois disciplines différente (linguistique, archéologie et ethnologie) apporte des perspectives nouvelles et un cadre robuste à l'identifications des emprunts. Basée sur le centre du Vanuat où il existe des chefferies à titre et des affiliations claniques, proches de ce que l'on trouve dans les sociétés polynésiennes, et oì l'on observe des emprunts polýcien du fait de la présence de conmentsús polynesien du fait de la présence de communautéc locutrices de langues polynéciennes à Mele, Ifira emae, cette étude indique donc des migration depuis des îles polynésiennes plusieurs siècles avan l'arrivée des Européens dans ces outliers.

Lattention particulière portée aux données collectées par Jean-Claude Rivierre, que les auteurs croisent avec leurs propres données, permet d'identifier les emprunts lexicaux aux langues polynésiennes et non polynésiennes en les rapprochant également des descriptions ethnographiques et des données archéologiques disponibles.

\section{Linguistique appliquée à l'enseignement des langues}

Les articles de Fabrice Wacalie et de Leslie Vandeputte illustrent, chacun à leur manière, l'importance de l'implication des chercheurs dans la formation des étudiants kanak et dans les politiques éducatives, o l'apprentissage des langues kanak (élaboration de documents pédagogiques pour les enseignants de langues kanak), etc., reprenant ainsi un sujet auxquels les Rivierre ont consacré une partie de leurs carrières. Dans son article, Fabrice Wacalie applique les travaux du premier terrain de Jean-Claude Rivierre sur l'aire linguistique drubéa-kapumë : le numèè (Goro et Touaourou), le kwênyii (île des Pins) et le drubéa (Païta et Unya), pour aboutir à des applications pédagogiques. La somme considérable de données que J.-C. Rivierre avait alors récoltées a donné lieu à plusieurs ouvrages importants, dont un sur la phonologie comparée des dialectes de l'extrême sud par en 1973 , ce qui fut l'objet de sa thèse de $3^{e}$ cycle. Et parmi ses travaux non publiés, il nous faut signaler le dictionnaire numèè et un corpus de textes oraux très important. Les travaux de Jean-Claude ont donc été le point de départ des recherches menées par Fabrice Wacalie dans le cadre de sa thèse. Jean-Claude fut aussi le précurseur des projets de valorisation des langues et cultures kanak de cette région comme de bien d'autres, sur lesquels Fabrice Wacalie revient ici. Il nous présente la façon dont les travaux de JeanClaude ont permis de développer l'enseignement des (acher langues du Sud par la creation d' outils pédagogiques puisant largement dans ses corpus, ce qui indique quel point les travaux de Jean-Claude - comme ceux de Françoise sur d'autres langues- ont été novateur et sont toujours d'actualité en Nouvelle-Calédonie.
Leslie Vandeputte, quant à elle, expose la manière dont il est possible de concilier le multilinguisme et les représentations des acteurs sociaux dans une politique linguistique éducative à partir du cas singulie du Vanuatu. Elle présente clairement la diversité de problèmes qui se posent lorsque l'on veut utiliser le bislama comme langue d'éducation. Le bislama es la lingua franca essentielle du Vanuatu et l'emblème fondamental de l'identité ni-Vanuatu ; il est reconnu comme langue nationale dans la constitution du Vanuatu. Malóé tout, il manque nécnstitution prestige par mapper aux deux la néanmoins de prostige par rappot aux de colonisation que sont le français et l'anglais. Après une première réforme juste après lindépendance, dans les années 1980, pour revoir les programmes scolaire en fonction d une politique linguistique éducative donnant une place au bislama, en 2016, le ministèr de l'Éducation a soutenu l'enseignement des langues vernaculaires durant les premières années de l'école primaire. Une soixantaine de programmes d'études en langues vernaculaires ont alors été promus. Dan la capitale Port-Vila, forcément multilingue, les en fants commencent l'école en bislama. Le cas présenté ici est tout à fait intéressant en comparaison de ce qui se passe en Nouvelle-Calédonie à propos des lanqui se passe nn Ne le gues kand'hui, à Nor

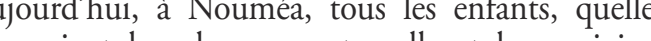
que soient leur langue maternelle et leur origine, kanak. Et cela ne plaît pas à tout le monde!

Idéologies linguistiques et confrontation au local

Dans la même lignée que celle tracée par les Rivierre, Christine Jourdan s interesse à la façon de faire un dictionnaire, à la dimension éthique du retour des données dans les pays où elles ont été collectées, ic les îles Salomon. Elle était venue au LACITO en 2001 pour présenter ce dictionnaire, un an avant sa publi- cation en 2002. Ce fut l'occasion pour elle de revoir les Rivierre qu'elle avait déjà croisés lors de congrès de linguistique océanienne et de discuter avec eux sur son expérience aux Salomon en regard de leur expéson experience aux Salomon en regard de leur expetance existant entre les données collectées et les dontance existant entre les données collectées et les don-
nées telles qu'elles sont typiquement restituées par les linguistes et les anthropologues, elle prend pour exemple le dictionnaire de pijin et montre comment les techniques de compilation d'un dictionnaire et les idéologies linguistiques de ses informateurs conjuguées aux siennes le façonnent et en font un produit loin des données originales. La dimension éthique d'une telle transformation doit donc être questionnée. Finalement, elle explique que son dictionnaire, déjà vieux de vingt ans, n’est utilisé par personne aux dejà vieux de ving ans, n'est

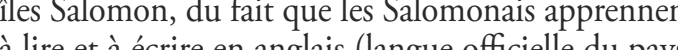
à lire et à ecrire en anglais (langue officielle du pays) et non en pijin. Par contre, les jeunes Salomonais présents sur les réseaux sociaux sont en train de déve-
lopper leur propre orthographe de cette langue, qui n'est ni du pijin ni de l'anglais.

On voit ici une différence très concrète avec les dictionnaires des langues kanak faits par les linguistes du LACITO qui furent tous pris en main très rapidement par les locuteurs des langues concernées, peutêtre en raison d'une histoire coloniale particulière en Nouvelle-Calédonie et de la revendication d'indépendance de ces cinquante dernières années.

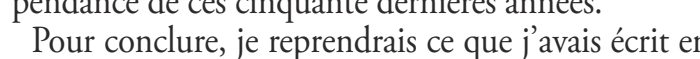
2018 dans l'in memoriam fait pour Jean-Claude :

"Partout où je suis passée pour mes recherches, la mémoire de Jean-Claude comme de Françoise était vivace et respectueuse tant ils ont marqué les gens avec lesquels ils ont travaillé et habité sur le terrain. Leur vie passionnée de chercheurs engagés auprès des Kanak et de leurs langues est digne de respect. " (2018b : 615)

\section{Remerciements}

Je remercie chaleureusement Nicolas Rivierre et sa famille pour nous avoir autorisé à publier ce article et pour les renseignements d'ordre familial et les photographies qu il nous a transmis et permis de reproduire ici ( $c f$. clichés notés CFR). Toute ma gratitude également aux nombreux collègues qui m’ont fourni compléments et photographies. Ils se reconnâtront. Enfin, je remercie Marie-Laure Bachere-Gouretneur, responsable du Pôle national de conservation des données et documents du CNRS, qui pilote sa podes donnees et documents du CNRS, qui pilote sa politique archivistique au niveau national, et le service
des archives du CNRS de Gif-sur-Yvette, tout particudes archives du CNRS de Gif-sur-Yvette, tout particulièrement Loïse Scherer (archiviste), pour leur aide dans la recherche des dossiers de carrière de Françoise Ozanne-Rivierre et Jean-Claude Rivierre (qu'ils avaient en leur possession) comme pour leur accueil. Ces données ont été complétées au LACITO par les dossiers encore conservés chez nous. Je remercie aussi, au LACITO, Balthazar Do Nascimento pour certaines photos qu’il a numérisées, Séverine Guillaume pour la liste des données sur pangloss ou à la BNF et Raphä̈lle Chossenot (également ISO) pour son aide dans l'établissement de la bibliographie de Françoise et Jean-Claude et s dans le texte.

Mes "remerciements " aussi à wikipedia où j'al rouvé de nombreuses précisions biographiques su les personnes citées.

\section{BIBLIOGRAPHIE}

Bensa Alban, 2018. In memoriam. Jean-Claude Rivierre (1938-2018), Journal de la Société des Océanistes 147 : 2018. La Kanaky Nouvelle-Calédonie rendez-vous avec l'histoire (I. Leblic et U. Cugola éds), pp. 618-620 (http://journals.openedition. eds), pp. 618-62
org/jso/9818).

Département OcÉanie du lacito, 2007. Hommage à Françoise Ozanne-Rivierre, Journal de la Société des Océanistes 125, p. 351 (http://journals. openedition.org/jso/1120).

Gaillard Gérald, 2004. The Routledge Dictionar of Anthropologists, New York, Routledge (1e éd. 1997. Le dictionnaire des ethnologues et des anthropologues, Paris, Armand Colin).

Haudricourt André-Georges, 1968. La langue de Gomen et la langue de Touho en Nouvelle-Calédonie, Bulletin de la société linguistique de Paris 63, 1 pp. 218-235 (rééd. 1972 in Problèmes de phonologie diachronique, Paris, SELAF, pp. 363-381).

Leblic Isabelle, 1993. Les Kanak face au développement. La voie étroite, Grenoble, Presses universitaires de Grenoble (avec le soutien de l'ADCK).

-, 2002. Ignames, interdits et ancêtres en NouvelleCalédonie, Journal de la Société des Océanistes 114115: En hommage à Jacques Barrau (C. Coiffie éd.), pp. 115-127 (http://journals.openedition. $\mathrm{org} / \mathrm{jso} / 1439)$.

-, 2010. Parcours en anthropologie maritime, en technologie, en anthropologie de la parenté et des rituels, de la Bretagne à la Nouvelle-Calédonie kanak, mémoire de synthèse pour la soutenance de l'habilitation à diriger les recherches, anthropologie sociale et ethnologie, soutenue le $20 \mathrm{mai}$ 2010 à l' врне-Paris (https://tel.archives-ouvertes. $\mathrm{fr} / \mathrm{tel}-00829158$ )

—, 2018a. Vivre de la mer, vivre avec la terre... en pays kanak. Savoirs et techniques des pêcheurs kanak du sud de la Nouvelle-Calédonie, Paris, Société des Océanistes, Travaux et documents océanistes 1.

-, 2018b. In memoriam. Jean-Claude Rivierre (1938-2018), Journal de la Société des Océanistes 147 : 2018. La Kanaky Nouvelle-Calédonie a rendez-vous avec l'histoire (I. Leblic et U. Cugola éds) pp. 613-617 (http://journals.openedition.org/ jso/9818). 
Leblic Isabelle et Bertrand Masquelier, 2020. Introduction. De l'ethnolinguistique à l'anthropologie linguis I pologie linguistique au LaCITO, in I. Leblic et . Souag (eds), Du terrain a la theorie : les 40 ans du LACITO, Villejuif, LACITO-Publications, Hor
série 1, pp. 15-34.

Osmond Meredith, 2009. Cat's cradle: A disappointing field for lexical reconstruction, in Alexander Adelar and Andrew Pawley (eds), Austroneshon historical linguistics and culture historn: a festschift historical linguistics and culture history: a festschrif for Robent Bhst, Canberra, ANu, Research School of Pacific and Asian Studies, Pacific Linguistic 601, pp. 509-514

PaWley Andrew, 1966. Polynesian Languages: A Subgrouping Based on Shared Innovations in Morphology, Journal of the Polynesian Society 75, 1, pp. 39-64 (http://archive.wikiwix.com/ cache/?url=http\%3A\%2F\%2Fwww.jps.auckland ac.nz\%2Fdocument $\% 2$ FVolume_75_1966\% 2FVolume_75\%2C_No._1\%2FPolynesian languages\%3A_a_subgrouping_based_on_shared_innovations_in_morphology\%2C_by_Andrew_Pawley\%2C__p_39_-_64\%2Fp1).

SAussol Alain, 2018. In memoriam. Jean-Claude Rivierre (1938-2018), Journal de la Société des Océa-

nistes 147 : 2018. La Kanaky Nowvelle-Calédonie a rendez-vous avec l'histoire (I. Leblic et U. Cugola éds), pp. 611-612 (http://journals.openedition. eds), pp. 611-61.
$\mathrm{org} /$ /so/9818).

SECRÉTARIAT GÉNÉRAL du COMitÉ NATIONAL, 2010 (juillet). Les sections et les commissions interdisciplinaires depuis 1949. Les intitulés et les motsclés, $25 \mathrm{p}$.

SouAG Lameen, 2020. Introduction. Linguistique de terrain, panchronie et typologie au LACTIO, in I. Leblic et L. Souag (éds), Du terrain à la théorie les 40 ans du LACITO, Villejuif, LACITO-Publications, Hors série 1, pp. 35-41.

\section{Archives consultées au service des archives du}

CNRS de Gif-sur-Yvette

RivierRe Jean-Claude (dossiers de carrière), 1963 1981, 910025 DPAA ; 1982-1989, 970073 SHS article 7 ; 1990-2003, 050048 SHS, article 11.

OzanNe-RIvierre Françoise (dossiers de carrière), 1967-1972, 910031 DPCF ; 1970-1981, 910025 DPAA; 1990-1999, 060037 HES, article 7 2000-2005, 090075 INSHS, article 19.

\section{ANNEXE}

\section{BIBLIOGRAPHIE DE FRANCOISE OZANNE-RIVIERRE}

\section{ET DE JEAN-CLAUDE RIVIERRE}

Françoise et Jean-Claude ayant réalisé certaines de leurs publications ensemble, j'ai pris le parti de faire une bibliographie commune pour éviter les répétitions. Outre la classification retenue distinguan mémoires, ouvrages et articles, cette présentation suit un ordre chronologique et alphabétique à l'intérieur de chaque rubrique.

\section{Mémoires et thèses}

Rivierre Jean-Claude, 1968. Phonologie et lexique de Tongoa, Paris, micro-édition du centre documentaire pour l'Océanie, $363 \mathrm{p}$.

Rivierre Jean-Claude, 1969a. Lexique de Futuna, Paris, micro-édition du centre documentaire pour l'Océanie, 83 p.

Rivierre Jean-Claude, 1969b. Phonologie et lexiques comparés des dialectes de Tanna, Paris, micro-édition du centre documentaire pour l'Océanie, 202 p.

Rivierre Jean-Claude, 1970. Phonologie comparée des dialectes de l'extrême sud de la NouvelleCalédonie, sous la dir. d'A. Martinet, Paris, EPHE, vol. (268 p., 3 cartes). [thèse de $3^{\mathrm{e}}$ cycle, de Lettres]

Ozanne-Rivierre Françoise, 1973. Le iaai, langue mélanésienne d'Ouvéa (Nouvelle-Calédonie), sous la dir. de B. Pottier, Paris 3 [thèse de $3^{e}$ cycle, de Lettres].

\section{Ouvrages personnels}

Rivierre Jean-Claude, 1973a. Phonologie compare des dialectes de l'extrême sud de la Nouvelle-Calédonie, Paris, Société d'études linguistiques et anthropologiques de France (SELAF), 214 p. (publication de la thèse soutenue en 1970).

Rivierre Jean-Claude, 1975. Grammaire de la langue cèmuhî, Paris, SELAF, 375 p.

Ozanne-Rivierre Françoise, 1976a. Le iaai, langue mélanésienne d'Ouvéa (Nouvelle-Calédonie). Phonologie, morphologie, esquisse syntaxique, Paris, Société d'études linguistiques et anthropologiques de France (sELAF), Tradition orale 20, $245 \mathrm{p}$.

Rivierre Jean-Claude, 1980. La langue de Touho. Phonologie et grammaire du cèmuhî (NouvelleCalédonie), Paris, Société d'études linguistiques et anthropologiques de France, 363 p.

Rivierre Jean-Claude, 1983. Dictionnaire paicî-français, suivi d'un lexique français-paicî, Paris, Société France, Langues et cultures du Pacifique 4, 375 p.

Ozanne-Rivierre Françoise, 1984. Dictionnaire iaai-français (Ouvéa, Nouvelle-Calédonie), suivi d'études linguistiques et anthropologiques de d'un lexique français-iaai, Paris, Société d'études linguistiques et anthropologiques de France (SELAF), Langues et cultures du Pacifique 6, $179 \mathrm{p}$.

Ozanne-Rivierre Françoise, 1986. Pue thawe: mythe en langue pije, recueilli par Françoise $\mathrm{Ri}-$ vière... auprès de $\mathrm{M}$. Timothée Daahma Le We ill. de Paula Boi, Nouméa, Office culturel, scientifique et technique canaque, $12 \mathrm{p}$., ill.

Ozanne-Rivierre Françoise, 1994a. L'orientation dans l'espace à travers les langues kanak, Nouméa ADCK, Cahiers des conférences de l'ADCK 2, $40 \mathrm{p}$.

Rivierre Jean-Claude, 1994a. Dictionnaire cèmuhîfrançais, suivi d'un lexique français-cèmuhî, Paris, Peeters, Langues et culture du Pacifique 9, 543 p.

\section{Ouvrages collaboratifs}

Thomas Jacqueline M.C., Simha Arom, JeanPierre Caprile, G. Dournon-Taurelle, Aurore Monod, Suzy Platiel, Jean-Claude Rrvierr et C. Venot, 1971. Questionnaires thématiques Enquête et description des langues à tradition oral 5, Paris, SELAF, $139 \mathrm{p}$.

Ozanne-Rivierre Françoise avec Poindi Tein, 1975. Textes nemi, Nouvelle-Calédonie, vol. 1 Kavatch et Tendo, Paris, SElaf, Tradition orale 31 , $316 \mathrm{p}$. ('introduction comporte une présentation phonologique et syntaxique).

Haudricourt André-Georges, Jean-Claude RIVIERRE, Françoise Ozanne-Rivierre, Claire Moyse-Faurie et Jacqueline de La Fontinelle 1979. Les langues mélanésiennes de Nouvelle-Calédonie, Nouméa, Direction de l'enseignemen catholique (DEC), Bureau psychopédagogique, Éveil 13, $105 \mathrm{p}$

Ozanne-Rivierre Françoise avec Poindi Tein, 1979. Textes nemi, Nouvelle-Calédonie, vol. 2 : Bas-Coulna et Haut Coulna, accompagné d'un lexique nemi-francais), Paris, SELAF, Traditio orale 32, 2 vol. (316 p. + 265 p. / lexique nem français $100 \mathrm{p}$.).

Rivierre Jean-Claude, Françoise Ozanne-Rivierre et Claire Moyse-FAurI 1980 . Mythes et contes de la Grande-Terre et des îles Loyauté (Nouvelle-Calédonie), Paris, Société d'études linguistiques anthropologiques de France (SELAF), LACITO-Documents, Asie-Austronésie 3, 225 p., carte.

Bensa Alban et Jean-Claude Rivierre, 1982. Les Chemins de l'alliance: L'organisation sociale et ses représentations en Nouvelle-Calédonie (région de Touho - aire linguistique cèmuhî), Paris, Société d'études linguistiques et anthropologiques de France, Langues et cultures du Pacifique 1, $586 \mathrm{p}$.

Ozanne-Rivierre Françoise avec André-Georges HaUdricourt, 1982. Dictionnaire thématique des langues de la région de Hienghène (NouvelleCalédonie): pije, fwâi, nemi, jawe, précédé d'une phonologie comparée des langues de Hienghène et du proto-océanien, Paris, SELAF, LACITO-Documents Asie-Austronésie 4, 285 p., 2 cartes.

Bensa Alban et Jean-Claude Rivierre, 1983. Histoire canaques, Paris, CILF, coll. fleuve et flamme, $160 \mathrm{p}$.

Ozanne-Rivierre Françoise et Martine MazauDON, 1986. Lexique nyâlayu (Balade), NouvelleCalédonie, Paris, LACito-CNRs, Notes et Documents, $94 \mathrm{p}$.

Hollyman Jym et Françoise Ozanne-Rivierre 1987. De muna fagauvea I, Dictionnaire fagauvea-français, Aukland, Linguistic Society of New Zealand, Te Reo monographs, $271 \mathrm{p}$.

Bensa Alban et Jean-Claude Rivierre (éds), 1995. Les filles du rocher Até: contes et récits paicî / [textes réunis par] Alban Bensa et Jean-Claude Rivierre ; les narrateurs, Barthélémy Bwérékëu, Cécile Göröipëwé, Clément Görömôtö... [et al.], Paris, Nouméa, Geuthner, ADCK, Patrimoine kanak de la Nouvelle-Calédonie 1.

OzanNe-Rivierre Françoise avec la collab. de B. \& S. Boiguivie et E. Dedane, 1998. Le nyelâyu de Balade (Nouvelle-Calédonie), Paris, Peeters, Langues et cultures du Pacifique 12, 276 p.

Bensa Alban et Jean-Claude Rivierre (éds), 1999. Le Pacifique: un monde épars. Introduction interdisciplinaire à l'étude de l'Océanie, Paris, L'Harmattan.

Rivierre Jean-Claude, Françoise Ozanne-Rivierre et Claire Moyse-Faurie (éds), 2005. Tâdo et crabe, d'après le conte "Crabe et Tâdo " dit par Célestin Tiemwaou, ill. par Ismaëlla Pourouda et Francia Boi, Koohnê (Nouvelle-Calédonie), province Nord, Direction de l'enseignement de formation et de l'insertion des jeunes, $36 \mathrm{p}$. (non paginé), ill. en coul.

RIVIERRE Jean-Claude, Sabine Ehrhart avec la collab. de Raymond DiéLA, 2006. Le bwatoo et les dialectes de la région de Koné (Nouvelle-Calédonie), Paris-Leuven, Peeters, coll. LCP 17, 502 p.

\section{Édition de volumes collectifs}

Bril Isabelle et Françoise Ozanne-Rivierre (eds) 2004. Complex predicates in Oceanic Languages: Studies in the Dynamics of Binding and Boundness, Berlin, Mouton de Gruyter (EALT 29), XI-395 p., carte.

\section{Articles de revue ou chapitres d'ouvrage}

RIVIERRE Jean-Claude, 1971. La tradition orale, in Enquêtes et description des langues à tradition orale, Paris, SELAF, pp. 95-107.

RIvierRe Jean-Claude, 1972a. À propos des ton de Nouvelle-Calédonie, in Hommages à André Georges Haudricourt, Paris, Klincksieck, tome 1 pp. 207-208.

Rivierre Jean-Claude, 1972b. Les langues d'Océanie, Encyclopaedia universalis, vol. 11, pp. 1052-1053. 
RivierRe Jean-Claude, 1972c. Les tons de la langue de Touho (Nouvelle-Calédonie) : étude diachronique, Bulletin de la Société de Linguistique de Paris 67 (1), pp. 301-316.

RIVIERRE Jean-Claude, 1973b. La nomenclature des coquillages en langue de Touho, Journal de la Société des Océanistes 39, pp. 139-150 (https://doi. org/10.3406/jso.1973.2422).

RivierRe Jean-Claude, 1974a. Tons et segments du discours en langue paicî (Nouvelle-Calédonie), Bulletin de la Société de Linguistique de Paris 69 (1), pp. 325-340.

RivierRe Jean-Claude, 1974b (noté à paraître dan rapport 1973). La langue cèmuhî, Les langues du monde, nlle éd., $22 \mathrm{p}$.

Ozanne-Rivierre Françoise, 1975a. La littérature orale nemi (Nouvelle-Calédonie), Journal de la Sociétédes Océanistes 49, pp. 399-434 (www.persee.fr/ doc/jso_0300-953x_1975_num_31_49_2725 https://doi.org/10.3406/jso.1975.2725).

Ozanne-Rivierre Françoise, 1975b. Phonologie du nemi (Nouvelle-Calédonie) et notes sur les consonnes postnasalisées, Bulletin de la Société de Linguistique de Paris 70/1, pp. 345-356.

Bensa Alban et Jean-Claude Rivierre, 1976. De quelques genres littéraires dans la tradition orale paicî (Nouvelle-Calédonie), Journal de la Sociéte des Océanistes 50, pp. 31-66 (https://journals.openedition.org/jso/persee-246523).

Rivierre Jean-Claude, 1978. Accents, tons et inversion tonale en Nouvelle-Calédonie, Bulletin de société de linguistique de Paris 73, 1, pp. 415-443.

Rivierre Jean-Claude (c. 1979a). Présentation générale des langues de Nouvelle-Calédonie, Manue d'alphabétisation dans les diverses langues de Nouvelle-Calédonie, Nouméa, DEC, 40 p.

RivierRe Jean-Claude (c. 1979b). Exposés et textes sur les langues tonales du centre et du sud, vol. 2 Nouméa, DEC, 60 p.

Rivierre Jean-Claude, (c. 1981). La politique linguistique en Nouvelle-Calédonie (32 p. dactylographiées), traduit en anglais par Noal Mellott, Yagl ambu. Revue linguistique de l'université de Port-Moresby (Papouasie Nouvelle-Guinée)

Rivierre Jean-Claude, 1981. Linguistique, Atlas de la Nouvelle-Calédonie et dépendances, Paris, ORsтом, pl. 19, 3 p.

Ozanne-Rivierre Françoise, 1982. Langues de Hienghène et proto-océanien : Phonologie comparée, in A.-G. Haudricourt et F. Ozanne-Rivierre, Dictionnaire thématique des langues de Ia région de Hienghène (Nouvelle-Calédonie): pije, fwâi, nemi, jawe, Paris, SELAF, LACITO-Documents Asie-Austronésie 4, pp. 9-61.

Moyse-Faurie Claire et Françoise Ozanne-RIVIERRE, 1983a. Langues à verbes initial et sujet marqué en Nouvelle-Calédonie, in A. Cartie (éd.), Typologie linguistique (journée d'études 5), Paris, Université René Descartes, UER de linguistique générale et appliquée, pp. 21-32.

Moyse-Faurie Claire et Françoise Ozanne-RiVIERRE, 1983b. Subject case markers and word order in New Caledonia and Loyalty Islands languages, in A. Halim, L. Carrington et S.A. Wurm (eds), Papers from the Third International Conference on Austronesian Linguistics 4. Thematic variation, Canberra, Australian National University, Research School of Pacific Studies, pp. 113-152.

Bensa Alban et Jean-Claude Rivierre, 1984. Jean Guiart et l'ethnologie, L'Homme 24 (1), pp. 101105 [Note critique en réponse à un CR sur « Les chemins de l'alliance »]).

Rivierre Jean-Claude, 1984. Publication de textes en langue paicî : poèmes, récits et contes, in Jè nye wii mwara paicî et Jè nye pi-cöö mwara paicî (livret e l'enseignant), Nouméa, CTRDP.

Rivierre Jean-Claude, 1985. La colonisation et les langues, Les temps modernes 464, pp. 1688-1717.

Ozanne-Rivierre Françoise, 1986. Redoublement expressif et dédoublement des séries consonantiques dans les langues des îles Loyauté (NouvelleCalédonie), Te Reo 29 : volume en hommage à K.J. Hollyman, Linguistic Society of New Zealand pp. 25-53.

Zzanne-Rivierre Françoise, 1987. L'expression linguistique de l'orientation dans l'espace : quelques exemples océaniens, Cahiers du LACITO. Revue d'ethnolinguistique 2, pp. 129-155.

Bensa Alban et Jean-Claude Rivierre, 1988a. De l'Histoire des mythes. Narrations et polémiques autour du rocher Até (Nouvelle-Calédonie), L'Homme 28 (106-107), pp. 263-295.

Bensa Alban et Jean-Claude Rivierre, 1988b. La portée pratique des mythes, NYX (revue littéraire trimestrielle) 5, pp. 33-41.

Ozanne-Rivierre Françoise, Jean-Claude Rivierre et Claire Moyse-Faurie, 1988. An 'École populaire kanak (EPK)' : the Canala experiment Interview with Marie-Adèle Jorédié (June 1987), in M. Spencer, A. Ward et J. Connell (eds), Essays in nationalism and dependancy, St Lucia, University of Queensland Press, pp. 198-218.

Ozanne-Rivierre Françoise, 1989. Le développement des voyelles nasales dans les langues du nord de la Nouvelle-Calédonie, Cahiers du LACITO. Revue d'Ethnolinguistique 4, pp. 83-100.

OzanNe-Rivierre Françoise et Jean-Claude RiVIERRE, 1989. Nasalization/oralization: Nasa vowel development and consonant shifts in New Caledonian languages, in R. Harlow and R. Hooper (eds), VICAL I: Oceanic Languages, Papers from the Fifth International Conference on Austronesian Linguistics, Auckland, Linguistic Society of New Zealand, pp. 413-432.
Bensa Alban et Jean-Claude Rivierre, 1990. Une poésie paicî : la mort du chef Céu, in De jade et de nacre. Patrimoine artistique kanak, Paris, Réunion des musées nationaux, pp. 189-205.

Moyse-Faurie Claire et Jean-Claude Rivierre, 1991. La recherche linguistique française dan le Pacifique (1960-1990), in F. Doumenge et J.-P. Doumenge (éds), Le Pacifique, l’océan, ses rivages et ses îles, Bordeaux-Monaco, CRET-Bordeaux III-Institut océanographique, pp. 396-402.

Ozanne-Rivierre Françoise, 1991a. Incorporation of genitive relators in the languages of New Caledonia and the Loyalty Islands, in R. Blust (ed.), Currents in Pacific linguistics: paper on Austronesian languages and ethnolinguistics in honour of George W. Grace, Canberra, ANu, Pacific Linguistics C-117, pp. 321-338.

Rivierre Jean-Claude, 1991a. Loss of final consonants in the North of New Caledonia, in R. Blust (ed.), Currents in Pacific linguistics: Papers on Austronesian languages and ethnolinguistics in hour of George W Gour of George W. Grace, Canberra, The Australian
National University, Pacific Linguistics C-117, pp. 415-432.

RIVIERRE Jean-Claude, 1991b. Les langues kanak, Bulletin de l'association pour l'evolution pacifique de la Nouvelle-Calédonie 6, pp. 3-6.

Moyse-Faurie Claire et Jean-Claude Rivierre 1992. Langues, in Nouvelles Calédonies d'avant 1914, collection Pacifique III, Le Mans, Paris, Association Pacifique, pp. 36-43.

Ozanne-Rivierre Françoise, 1992. The ProtoOceanic consonantal system and the languages of New Caledonia, Oceanic Linguistics 31 (2), pp. 191-208.

RIVIERRE Jean-Claude, 1992. Une lettre du père Montrouzier, in Nouvelles-Calédonies d'avant 1914. collection Pacifique III, Le Mans, Paris, Association Pacifique, pp. 37-43.

Rivierre Jean-Claude, 1993. Tonogenesis in New Caledonia, in J.A. Edmondson and K.J. Gregerson (eds), Tonality in Austronesian Languages, Honolulu, University of Hawai i, Oceanic Linguistics Special Publication 24, pp. 155-173.

Ozanne-Rivierre Françoise, 1994b. Iaai loanwords and phonemic changes in Fagauvea, in T. Dutton et D.T. Tryon (eds), Language contact and change in the Austronesian world, Berlin, New York, Mouton de Gruyter, pp. 523-550

Ozanne-Rivierre Françoise, 1994c. L'expression de la personne : quelques exemples océaniens, Fait de langues 3 : La personne, pp. 211-219.

Ozanne-Rrvierre Françoise, 1994d. Diasystème fidjien et dialectologie structurale, in Catherin Paris (éd.), Les cahiers du LACITO 8, pp. 7-24.

Rivierre Jean-Claude, 1994b. Contact-Induced Phonological Complexification in New Caledonia, in T. Dutton et D.T. Tryon (eds), Language contact and change in the Austronesian world (dan le cadre du vol. 2 de l'Intercontinental Distionary pp. 497-522.

Ozanne-Rivierre Françoise, 1995a. Nemi, in Darrell T. Tryon, Malcom D. Ross, Charles E. Grimes, Adrian Clynes and K.A. Adelaar (eds), Comparative Austronesian Dictionary. An Introduciton to Austronesian Studies, part 1. fasc. 2, Berin, Mouton de Gruyter, Trends in Linguistics 10, pp. 849-854.

OzanNe-RivierRe Françoise, 1995b. Structural changes in the languages of Northern New Caledonia, Oceanic Linguistics 34/1, pp. 45-72.

Rivierre Jean-Claude, 1995. Cèmuhî, in Darrell T. Tryon, Malcom D. Ross, Charles E. Grimes, Adrian Clynes and K.A. Adelaar (eds), Comparative Austronesian Dictionary. An Introduciton to Austronesian Studies, part 1. Fasc. 2 Berlin, Mounde Gruyter, Trends in Linguistics 10, pp. 855-858.

OzanNe-Rivierre Françoise et Jean-Claude RIVIERRE, 1996. Modèles panchroniques : l'exemple des consonnes postnasalisées, Revue de phonétiqu appliquée 121 : Changements phonétiques (Dipp. 247-262.

RIVIERRE Jean-Claude, 1996a. Mythistoire et archéologie dans le Centre-Vanuatu. L'histoire de Matanauretong (Tongoa), in M. Julien et al. (éds), Mémoire de pierre, mémoire d'homme : tradition et archéologie en Océanie : hommage à José Garanger Paris, Publications de la Sorbonne, Homme et Société 23, pp. 431-463.

Rivierre Jean-Claude, 1996b. André-Georges Haudricourt (1911-1996), Journal de la Société des Oćamistes 103, pp.317-318 (www.perseeff/doc/ jso 0300-953x_1996 num_103_2_2003).

OzANNE-RIvierre Françoise, 1997a. Systèmes d'orientation : Quelques exemples austronésien in C. Fuchs et S. Robert (éds), Diversité des langues et representations cognitives, Paris, Ophrys, pp. 81-92.

Ozanne-Rivierre Françoise, 1997b. Spatial references in New Caledonien languages, in G. Senft (ed.), Referring to space: studies in Austranesian and Papuan Languages, Oxford, Clarendon Press, pp. $83-100$

Ozanne-Rivierre Françoise et Jean-Claude RIVIERRE, 1997a. In memoriam: André-Georges Haudricourt 1911-1996, Oceanic Linguistics 36/1, pp. 1-5.

RivierRe Jean-Claude, 1997. Labiovelar consonants in New Caledonia. Paper presented to the Third International Conference on Oceanic Linguistics, Hamilton, New Zealand.

Moyse-Faurie Claire et Françoise Ozanne-RIVIERRE Françoise, 1999. Negation in New Caledonia and Loyalty islands languages, in E. Hod- 
vhaugen et $\mathrm{U}$. Mosel (eds), Negation in Oceanic languages: Typological studies, München, Lincom Europa, pp. $57-79$.

OzANNe-Rivierre Françoise, 1999a. Spatial orientation in some Austronesian languages, in C. Fuch et S. Robert (eds), Language diversity and cognitive representations, Amsterdam/Philadephia, John Benjamins, pp. 73-84.

Ozanne-Rivierre Françoise, 1999b. Diasystème fidjien et dialectologie structurale, Cahiers $d u$ LACITO. Revue d'Ethnolinguistique 8 : Diasystème et longue durée, pp. 81-100.

Ozanne-Rivierre Françoise, 1999c. Haudricourt " côté linguiste ", Techniques et culture 33, pp. 189-195.

OzanNe-Rivierre Françoise, 1999d. Langues d'Océanie et histoire, in Alban Bensa et JeanClaude Rivierre (éds), Le Pacifique: un monde épars. Introduction interdisciplinaire à l'étude de l'Océanie, Paris, L'Harmattan, pp. 75-104.

Ozanne-Rivierre Françoise, 2000a. Terminologie de parenté proto-océanienne: Continuité et changement dans les langues kanak, in A. Bens et I. Leblic (éds), En pays kanak: Ethnologie, linquistique, archéologie, histoire de la Nouvelle-Calédonie, Paris, Maison des sciences de l'homme, pp. 69-100.

RivierRe Jean-Claude, 2000a. André-Georges Haudricourt et la phonologie: la phonologie panchronique en perspective, in Modèles linguistiques 40 , tome $\mathrm{xx}$, fasc. 2, pp. 9-20

RIVIERRE Jean-Claude, 2000b. La connaissance du malais et des langues de l'Océanie, in S. Auroux E.F.K. Koerner et al. (éds), Histoire des sciences $d u$ langage 1, 1, pp. 998-1001.

Jacobson Michel, Boyd Michailovskr, Françoise Ozanne-Rivierre et Jean-Claude RivierRe, 2001. Documentation des langues et tradition orale : le programme Archivage du LACITO, Séance II : Protocoles de collaboration entre anthropologues, populations autochtones et musées: exemples francais, in Symposium "Identités autochtomes: Paroles écrits et nouvelles technol on (15-18 Paroles, ecrits et nouvelles technologiques" (15-18 mai 2001), Pais, unEsco, pp. 20-22 (https:// fre?.pos.nSet=7\&queryId=e124eeda-07fd-4b80 fre?posInSet=7\&quer
aba7-c88ec4535323).

aba7-c88ec4535323). Some shared developments in pronouns in languages of Southern Oceania, Oceanic Linguistics 40 (1), pp. 33-66.

RivierRe Jean-Claude, 2001a. Écriture (en Océani insulaire), in C. Laux (éd.), 101 mots pour comprendre le Pacifique, Nouméa, éd. Île de Lumière.

Rivierre Jean-Claude, 2001b. Tonogenesis and evolution of the tonal systems in New Caledonia, the example of cèmuhî, in Shigeki Kaji (ed.), Proceedings of the symposium Cross-linguistic studies of to- nal phenomena, Tonogenesis, Japanese Accentology, and other Topics (Tokyo, 12-14 décembre 2000),

Tokyo, University of Foreign Studies, pp. 23-42.

Ozanne-Rivierre Françoise, 2003a. Laire coutumière iaai, in B. Cerquiligni (éd.), Les langues de France, Paris, PUf, pp. 421-426.

Ozanne-Rivierre Françoise, 2003b. Naissance de 'Union calédonienne, Journal de la Société des Océanistes 117 : Nouvelle-Calédonie, 150 ans apres la prise de possession (I. Leblic éd.), pp. 313-315 (http://journals.openedition.org//jso/1336).

RivierRe Jean-Claude, 2003. Langues de NouvelleCalédonie : Introduction, aires coutumières hoot ma waap, paici-camuki, ajie-aro, djubea-kapone, nengone, in B. Cerquiglini (éd.), Les langues de France, Paris, Puf, pp. 346-362, 365-404, 413420, 431-435.

OzanNe-Rivierre Françoise, 2004a. Spatial deixis in Iaai (Loyalty Islands), in G. Senft (ed.), Spatial deixis in Oceanic languages, Canberra, Austraian National University, coll. Pacific Linguistics, pp. 127-137.

Ozanne-Rivierre Françoise, 2004b. The evolution of the verb 'take' in New Caledonian languages, in I. Bril et F. Ozanne-Rivierre (eds), Complex Predicates in Oceanic Languare: Studies in the Dymmics of Binding and Boundness, Berlin, Mouton de Gruyter, pp. 331-346.

Ozanne-Rivierre Françoise et Jean-Claude RIVIERRE, 2004a. Évolution des formes canoniques dans les langues de Nouvelle-Calédonie, Faits de langues 24 : Les langues austronésiennes (E. Zeitoun éd.), pp. 141-153.

Ozanne-Rivierre Francoise et Jean-Claude RIVIERRE, 2004b. Un changement récurrent dans l'aire austronésienne, in E. Motte-Florac et G. Guarisma (éds), Du terrain au cognitif: Linguistique, ethnolinguistique, ethnosciences. À Jac queline M.C. Thomas, Paris, Peeters, pp. 197-209.

Ozanne-Rivierre Francoise et Jean-Claude RIVIERRE, 2004c. Verbal compounds and lexical prefixes in the langurges of New Caledonia, in Bril et F Ozanne-Rivierre (eds), Complex Premuts mics of Binding and Boundness, Berlin, Mouton de Gruyter, pp. 347-371

Ozanne-Rivierre Françoise, 2011. Le iaai, in E. Bonvini, J. Busuttil et A. Peyraube (éds), Dictionnaire des langues, Paris, Presses universitaires de France, coll. Quadrige - Dicos Poche), pp. 1230-1238.

RIVIERRE Jean-Claude, 2011. André-Georges Haudricourt et la phonologie : la phonologie panchronique en perspective, Le Portique [En ligne] 27 (https:/ journals.openedition.org/leportique/2534).

Moyse-Faurie Claire, Jacques Vernaudon et JeanClaude Rivierre, 2012. Les langues kanak, in Atlas de la Nouvelle-Calédonie, Nouméa, Insti- tut de recherche pour le développement, pl. 26, pp. 119-122.

\section{Comptes rendus d'ouvrage}

Ozanne-Rivierre Françoise, 1972. Compte rendu de The String Figures of Nauru Island (de H. Maude, 1971, Adelaide, Libraries Board of South Australia), Journal de la Société des Océanistes 36, pp. 317-318.

Ozanne-Rivierre Françoise, 1976b. Compte rendu de Manuel de tahitien moderne (de P. Prévost, 1975, Tahiti, Polytram Tipaerui), Journal de la Société des Océanistes 50, p. 129

Ozanne-Rivierre Françoise, 1976c. Compte rendu de Place Names of Hawaii (de M. Kawena Pukui, S.H. Elbert et E.T. Mookini, 1974, Honolulu, University Press), Journal de la Société des Océanistes 50, p. 129

Ozanne-Rivierre Françoise, 1976d. Compte rendu de New Guinea Diaries 1871-1883 (de M. Maclay, 1975, Madang (PNG), Kristen Press), Journal de la Société des Océanistes 50, p. 129.

Ozanne-Rivierre Francoise, 1978. Compte rendu de Aspects of Proto-Polynesian Syntax (de R. Clark, 1976), BSL 73, 2, pp. 444-447.

Ozanne-Rivierre Françoise, 1981. Compte rendu de Le Palawan (Philippines), phonologie, catégories, morphologie (de N. Revel-MacDonald, 1979 Paris, SELAF, ASEMI 4), Archipels 22, pp. 221-222.

Cartier Alice, Françoise Ozanne-Rivierre et JeanClaude Rivierre, 1982. Compte rendu du Troisième congrès international de linguistique indonésienne (Denpasar, Bali, janvier 1981), Archipels 24, pp. 31-39.

Ozanne-Rivierre Françoise, 1998a. Compte rendu de Grammaire du futunien (de C. Moyse-Faurie, Nouméa, 1997), Journal de la Société des Océa nistes 107 , p. 244.

OzanNe-Rrvierre Françoise, 2000b. Compte rendu de Grammaire du futunien (de C. Moyse-Faurie, Nouméa 1997. Centre de documentation pédagogique), Bulletin de la Société de Linguistique de Paris 95 (2), pp. 226-298.

\section{Rapports non publiés}

Ozanne-Rivierre Françoise, 1983. L'orientation dans l'espace et son expression dans les langues océaniennes, L'expression linguistique de l'espace dans les langues austronésiennes, ATP A651-3152, pp. 6 -33.

pp. 6 - 33.
Riverre Jean-Claude, Claire Moyse, Françoise OzAnNe-RIvierRe et Isabelle BrIL, 2001-2002. Notice sur les langues de Nouvelle-Calédonie destination de la DGLF, $80 \mathrm{p}$.
Capo Manon et Jean-Claude Rivierre, 2012. Trois études de manuscrits en langues kanak (paicî, cèmûhî, ajië), rapport final du programme " $\mathrm{Ar}$ chivage et valorisation d'un corpus de manuscrits kanak, une approche génétique ».

\section{Médias}

Un Cédérom réalisé pour le Centre culturel Tjibaou par Françoise Ozanne-Rivierre, à partir de textes enregistrés à Kaavatch, Tendo, Bas-Coulna et Ouaième, dans les années 1970 : quinze textes nemi (1 heure), coproduction CNRS/ADCK (en ligne sur Pangloss).

OzAnNe-RivierRe Françoise, 1998b. Quinze textes nemi (Nouvelle-Calédonie), cédérom pour le centre culturel Tjibaou, coproduction CNRS/ADCK.

Ozanne-Rivierre Françoise, 1998c. Lorigine de la monnaie traditionnelle kanak, texte en langue pije (Nouvelle-Calédonie), in J.-C. Rivierre (éd.), cédérom mixte, pour le centre culturel Tjibaou, coproduction CNRS/ADCK.

RIVIERRE Jean-Claude, 1998a. Littérature orale cèmuhî (Nouvelle-Calédonie), cédérom pour le Centre culturel Tjibaou, coproduction CNRS/ADCK.

RivierRe Jean-Claude, 1998b. Littérature orale de l'aire drubez-kapone (Nouvelle-Calédonie), cédérom pour le Centre culturel Tjibaou, coproduction CNRS/ADCK.

Rivierre Jean-Claude, 1998c. Textes en langues kwênyii, paicî et cèmuhî (Nouvelle-Calédonie), cédérom pour le Centre culturel Tjibaou, coproduction CNRS/ADCK.

\section{Manuscrits}

Rivierre Jean-Claude, nd. (c. 1994). Lexique havekefrançais, drubea-français, numèe-français, n.p.

RIVIERRE Jean-Claude avec la collaboration de Clément Vendégou, 1997. Lexique numèe-francais, n.p. (https://halshs. archives-ouvertes.fr/ halshs-02916886/document)

\section{Archives orales}

BNF - Département Archives et manuscrits, Enregistrements en langue cèmuhî, cote $\mathrm{DO}$ archivesetmanuscrits.bnf.fr/ark:/12148/ cc102782h/ca19912667).

PAngloss : 261 enregistrements, en majorité en cèmuhî, mais aussi en bwato, nemi, pijé, iaai, fagauvea... déposés par Jean-Claude dont 73 annotés en xml ou pdf (https://lacito.cnrs.fr/pangloss/ corpus/search.php?term=jean-claude+rivierre\&p articipant $={ }^{*} \&$ langue $=*$ \&type $=$ audio) et détaillés dans le document annexe accessible en ligne dans le dossier accompagnant ce volume. 

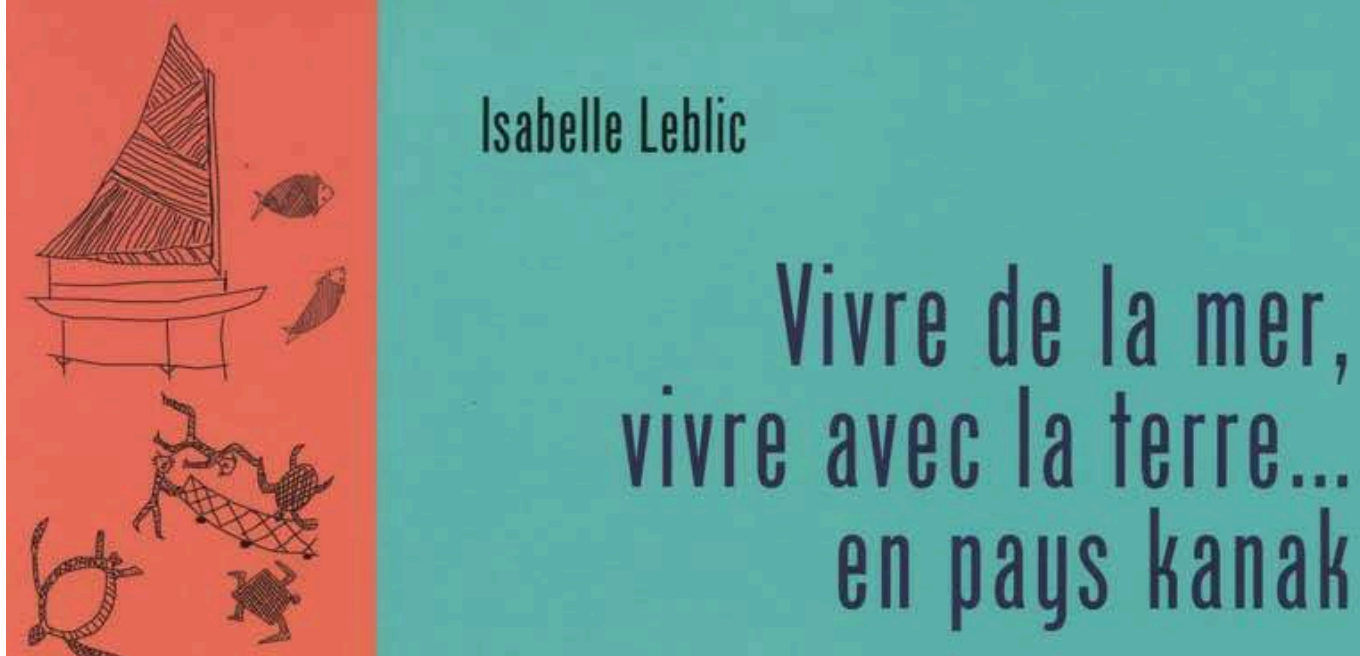

Savoirs et techniques des pêcheurs kanak du sud de la Nouvelle-Calédonie
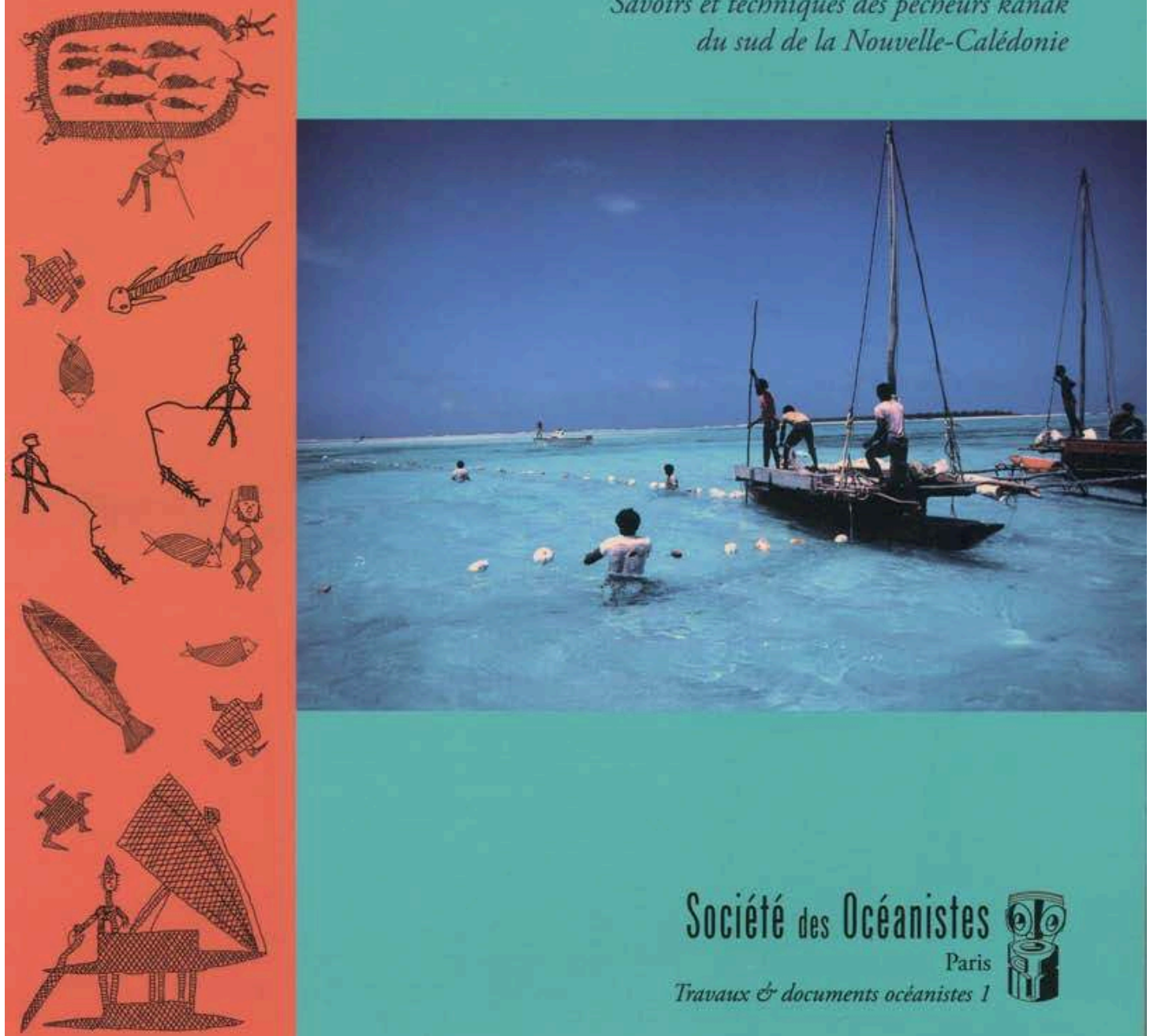

Paru en 2008 (288 pages, bibliographie, glossaires, index, plus de 600 illustrations en noir et blanc ou en couleur), cet ouvrage est disponible en version imprimée sur http://www.oceanistes.org/oceanie/les-editions-de-la-sdo/travaux-et-documents-oceanistes/ et version en ligne sur https://books. openedition.org/sdo/594 